JOURNAL OF THE AMERICAN MATHEMATICAL SOCIETY

Volume 20, Number 4, October 2007, Pages 1135-1186

S 0894-0347(07)00572-3

Article electronically published on May 11, 2007

\title{
RATIONAL POINTS ON COMPACTIFICATIONS OF SEMI-SIMPLE GROUPS
}

\author{
JOSEPH SHALIKA, RAMIN TAKLOO-BIGHASH, AND YURI TSCHINKEL
}

\section{INTRODUCTION}

Let $F$ be a number field and $\mathrm{G}$ a connected linear algebraic group over $F$. We are interested in distribution properties of rational points on smooth projective equivariant compactifications $X$ of $\mathrm{G}$ with respect to heights. More precisely, we assume that $X$ contains $\mathrm{G}$ as a Zariski open subset and that the left and right action of $\mathrm{G}$ on itself extends to $X$. Equivalently, $X$ is an equivariant compactification of $\mathrm{G} \times \mathrm{G} / \mathrm{G}$. A split over $F$ semi-simple group $G$ of adjoint type has a canonical (wonderful) compactification, constructed over an algebraically closed field in [16] and over arbitrary fields (and over $\mathbb{Z}$ ) in 40 and 17 . In this paper we consider wonderful compactifications of forms of such groups. One of our main results is a proof of Manin's conjecture for this class of varieties:

Theorem 1. Let $X$ be the wonderful compactification of a semi-simple group $G$ over $F$ of adjoint type and $\mathcal{L}=\left(L,\|\cdot\|_{v}\right)$ an adelically metrized line bundle such that its class $[L]$ in the Picard group $\operatorname{Pic}(X)$ of $X$ is contained in the interior of the cone of effective divisors $\Lambda_{\mathrm{eff}}(X) \subset \operatorname{Pic}(X)_{\mathbb{R}}$. Then

$$
\mathcal{Z}(\mathcal{L}, s):=\sum_{x \in G(F)} H_{\mathcal{L}}(x)^{-s}=\frac{c(\mathcal{L})}{(s-a(L))^{b(L)}}+\frac{h(s)}{(s-a(L))^{b(L)-1}}
$$

and, consequently,

$$
\mathcal{N}(\mathcal{L}, B):=\#\left\{x \in \mathrm{G}(F) \mid H_{\mathcal{L}}(x) \leq B\right\} \sim \frac{c(\mathcal{L})}{a(L)(b(L)-1) !} B^{a(L)} \log (B)^{b(L)-1}
$$

as $B \rightarrow \infty$. Here

- $a(L)=\inf \left\{a \mid a[L]+\left[K_{X}\right] \in \Lambda_{\mathrm{eff}}(X)\right\}$ (where $K_{X}$ is the canonical line bundle of $X)$;

- $b(L)$ is the (maximal) codimension of the face of $\Lambda_{\mathrm{eff}}(X)$ containing $a(L)[L]$ $+\left[K_{X}\right]$

- $c(\mathcal{L}) \in \mathbb{R}_{>0}$ and

Received by the editors February 10, 2006.

2000 Mathematics Subject Classification. Primary 14G05, 11G50; Secondary 11F70.

Key words and phrases. Manin's conjectures, rational points, wonderful compactifications, automorphic forms.

The second author was partially supported by the Clay Mathematics Institute and the NSA.

The third author was partially supported by NSF grants 0100277 and 0602333 . 
- $h(s)$ is a holomorphic function in the domain $\Re(s)>a(L)-\epsilon$, for some $\epsilon>0$.

Moreover, $c\left(-\mathcal{K}_{X}\right)$ is the constant defined by Peyre in $[30$.

We actually prove a more precise theorem with rate estimates; cf. Theorem 9.2 , The proof is based on the study of analytic properties of the height zeta function (0.1). The zeta function is first realized as a special value of an automorphic form on the group $G(\mathbb{A})$. Then we write down the automorphic Fourier expansion in terms of a basis of automorphic forms. In general, we also have a contribution from the continuous spectrum. The Fourier expansion gives an identity of continuous functions which we use to prove a meromorphic extension of the height zeta function.

This paper is part of a program, initiated in [19] and developed in [4, 30], 7], to relate asymptotics of points of bounded height to geometric and arithmetic invariants of $X$. For further results and motivating examples we refer to the book 31] and the papers [6], 38, [15] and [32]. An alternative proof of the asymptotic formula (0.2), based on adelic mixing and volume asymptotics implicit in Theorem 7.1, was given in [21].

The paper is organized as follows. In Part I we collect various results on algebraic groups and automorphic forms. Section 2 contains a theorem about transfer of onedimensional automorphic representations among inner forms of a group. Section 3.1 is concerned with Eisenstein series and automorphic Fourier expansions. The key results are Lemma 3.1 and Proposition 3.5. In Section 4.1 we recall a theorem of $\mathrm{Oh}$ (Theorem 4.2) which we use for estimates in the spectral expansion. In Part II we apply this theory to Manin's conjecture. In Section 5 we review the construction of wonderful compactifications and their geometric properties. In Section [6 we study height functions and their integrals in the local situation. The problem of regularizing global height integrals is considered in Section 7 In Section 8 we establish analytic properties of height zeta functions in several complex variables. Tauberian theorems imply asymptotics for the number of rational points of bounded height.

\section{Part 1. Algebraic groups and automorphic forms}

\section{Algebraic groups}

1.1. Basic notation. For a number field $F$, let $\operatorname{Val}(F)$ be the set of all places and $S_{\infty}=S_{\infty}(F)$ the set of archimedean places. For $v \in \operatorname{Val}(F)$, let $F_{v}$ be the completion of $F$ with respect to $v, \mathcal{O}_{F}$ (resp. $\mathcal{O}_{v}$ ) the ring of integers in $F$ (resp. $F_{v}$ ) and $q=q_{v}$ the order of the residue field $\mathbf{k}=\mathbf{k}_{v}$ of $\mathcal{O}_{v}$. For any finite set of places $S$ (containing $S_{\infty}$ ) we denote by $\mathcal{O}_{S}$ the ring of $S$-integers of $F$. We denote by $\mathbb{A}$ the ring of adeles, by $\mathbb{A}_{S}=\prod_{v \notin S}^{\prime} F_{v}$ and by $\mathbb{A}_{f}=\mathbb{A}_{S_{\infty}}$. Let $\Gamma_{F}$ and $\Gamma_{v}$ be the Galois groups of $\bar{F} / F$ and $\bar{F}_{v} / F_{v}$, respectively. For $L / F$ a Galois extension, with $w \in \operatorname{Val}(L)$ and $v \in \operatorname{Val}(F)$ satisfying $w \mid v$, set $\Gamma_{w / v}=\operatorname{Gal}\left(L_{w} / F_{v}\right)$.

For $X=X_{F}$ an algebraic variety over $F$ and $L / F$ an extension write $X_{L}=$ $X \times{ }_{F} L$ for the base-change to $L$ and $X(F)$ for the set of $F$-rational points of $X$. For $v \in \operatorname{Val}(F)$, we abbreviate $X_{v}=X_{F_{v}}$. We typically identify $X_{v}\left(F_{v}\right)$ and $X\left(F_{v}\right)$.

We denote by $\operatorname{Pic}(X)$ the Picard group of $X$ and by $\Lambda_{\text {eff }}(X) \subset \operatorname{Pic}(X)_{\mathbb{R}}$ the (closed) cone of effective divisors on $X$. We often identify line bundles, divisors 
and their classes in $\operatorname{Pic}(X)$. If $X$ has an action by an algebraic group $\mathrm{H}$ we write $\mathrm{Pic}^{\mathrm{H}}(X)$ for the group of isomorphism classes of $\mathrm{H}$-linearized line bundles on $X$.

1.2. Setup. Throughout this paper, $G$ is a fixed connected semi-simple group of adjoint type defined over a number field $F$ and $G^{\prime}$ a quasi-split group over $F$ of which $G$ is an inner form. The groups $G$ and $G^{\prime}$ have a unique split $F$-form $G^{s p}$. We may assume that $\mathrm{G}^{s p}$ is obtained by base-change from a split group, again denoted $\mathrm{G}^{s p}$, over $\mathbb{Q}$. We fix a Galois extension $E / F$ such that $\mathrm{G}(E)=\mathrm{G}^{\prime}(E)=\mathrm{G}^{s p}(E)$ identified as subgroups of $\mathrm{G}(\bar{F})$. Its Galois group is denoted by $\Gamma=\operatorname{Gal}(E / F)$. We denote the simply-connected cover of $\mathrm{G}$ by $\mathrm{G}^{s c}$. Let $\mathrm{T}$ be a maximal torus of $\mathrm{G}$ viewed as an algebraic group over $F$. For each $v \in \operatorname{Val}(F)$, we denote by $\mathrm{S}_{v}$ the maximal $F_{v}$-split subtorus of $\mathrm{T}$ considered as an algebraic group over $F_{v}$. The corresponding objects for $\mathrm{G}^{\prime}$ and $\mathrm{G}^{s p}$ are written $\mathrm{T}^{\prime}, \mathrm{S}_{v}^{\prime}$, and $\mathrm{T}^{s p}$, respectively; for $\mathrm{G}^{s p}$, we make the choice in such a way that $\mathrm{T}^{s p}$ is split. We choose maximal tori $\mathrm{T}, \mathrm{T}^{\prime}, \mathrm{T}^{s p}$ in such a way that they have the same set of rational points over $E$. In particular, $\mathrm{T}^{\prime}$ is split over $E$. We let $\mathrm{S}^{\prime}$ be the maximal split torus in $\mathrm{G}^{\prime}$ contained in $\mathrm{T}^{\prime}$. We choose a finite set $S_{E} \subset \mathrm{Val}(E)$ containing all the archimedean places so that if $w \notin S_{E}$ is non-archimedean, then

- the extension $E_{w} / F_{v}$ is unramified for all $v \in \operatorname{Val}(F)$ with $w \mid v$;

- if $v \in \operatorname{Val}(F)$ is not divisible by any $w \in S_{E}$, then $\mathrm{G}$ is quasi-split over $F_{v}$ and $\mathrm{G}\left(F_{v}\right)=\mathrm{G}^{\prime}\left(F_{v}\right)$

We will denote by $S_{F}$ the collection of all places of $F$ which are divisible by some $w \in S_{E}$. Later, we will need to enlarge $S_{E}$, and $S_{F}$, to satisfy extra assumptions; cf. Section 1.4. We have a standard and a twisted action of $\Gamma=\operatorname{Gal}(E / F)$ on $\mathrm{G}^{\prime}(E)$, denoted by

$$
g \mapsto \sigma(g), \quad \text { resp. } \quad g \mapsto \tilde{\sigma}(g)
$$

Here

$$
\tilde{\sigma}=c(\sigma) \cdot \sigma,
$$

where $c \in Z^{1}\left(\Gamma, \operatorname{Inn}\left(G^{\prime}\right)(E)\right)$ is a cocycle with values in the group of inner automorphisms of $\mathrm{G}^{\prime}$.

Pick a Borel subgroup $B^{s p}$ in $\mathrm{G}^{s p}$ which contains $T^{s p}$. As $\mathrm{G}^{\prime}$ is quasi-split it contains a Borel subgroup $\mathrm{B}^{\prime}$, containing $\mathrm{T}^{\prime}$, which is defined over $F$. If $v \notin S_{F}$, then by transfer of structure we obtain a Borel subgroup $\mathrm{B}\left(F_{v}\right)$ in $\mathrm{G}\left(F_{v}\right)$. If $v \in S_{F}$, we pick a minimal parabolic $F_{v}$-subgroup $\mathrm{B}\left(F_{v}\right)$ of $\mathrm{G}\left(F_{v}\right)$ containing $\mathrm{S}\left(F_{v}\right)$. For each place $v$, denote by $\tilde{\Phi}\left(\mathrm{G}\left(F_{v}\right), \mathrm{S}\left(F_{v}\right)\right)$ the set of roots of $\mathrm{S}\left(F_{v}\right)$ in $\mathrm{G}\left(F_{v}\right)$ and by $\Phi\left(\mathrm{G}\left(F_{v}\right), \mathrm{S}\left(F_{v}\right)\right)$ the set of non-multipliable roots in $\tilde{\Phi}$ with the ordering given by $\mathrm{B}\left(F_{v}\right)$. Let $\mathfrak{X}^{*}\left(\mathrm{~S}\left(F_{v}\right)\right)$ denote the set of characters of $\mathrm{S}\left(F_{v}\right)$ defined over $F_{v}$. Denote by $\mathfrak{X}^{+}$(resp. $\left.\Phi^{+}\right)$the set of positive characters (resp. roots) in $\mathfrak{X}^{*}\left(\mathrm{~S}\left(F_{v}\right)\right.$ ) (resp. $\Phi\left(\mathrm{G}\left(F_{v}\right), \mathrm{S}\left(F_{v}\right)\right)$ ) with respect to that ordering. We also let $\Delta\left(\mathrm{G}^{s p}, \mathrm{~T}^{s p}\right)=$ $\left\{\alpha_{1}, \ldots, \alpha_{r}\right\}$ be the set of simple roots of for the pair $\left(\mathrm{G}^{s p}, \mathrm{~T}^{s p}\right)$ with respect to the ordering introduced by $\mathrm{B}^{s p}$, and let $\rho$ be half the sum of the positive roots for the same pair. For any subset $I \subset[1, \ldots, r]$ we denote by $\mathrm{P}_{I}^{s p}$ the corresponding parabolic subgroup of $\mathrm{G}^{s p}$ and by $\mathrm{L}_{I}^{s p}$ its Levi subgroup. Let $\mathrm{W}$ be the Weyl group of the pair $\left(\mathrm{G}^{s p}, \mathrm{~T}^{s p}\right)$. Denote by $\left\{\mathrm{s}_{1}, \ldots, \mathrm{s}_{r}\right\}$ the set of simple reflections, by $\ell$ the length function on $\mathrm{W}$ and by $\mathrm{w}_{0} \in \mathrm{W}$ the longest element with respect to $\ell$. We let $\left\{\omega_{1}, \ldots, \omega_{r}\right\}$ be the collection of the fundamental weights of the simply-connected cover of $G^{s p}$. We will also occasionally speak of the root system of the pair $\left(G^{\prime}, T^{\prime}\right)$, 
the ordering given by the choice of $\mathrm{B}^{\prime}$, and the collection of simple roots $\Delta\left(\mathrm{G}^{\prime}, \mathrm{T}^{\prime}\right)$; the collection of $\Gamma_{F}$-orbits in $\Delta\left(\mathrm{G}^{\prime}, \mathrm{T}^{\prime}\right)$ is the same as $\Delta\left(\mathrm{G}^{\prime}, \mathrm{S}^{\prime}\right)$.

If $v$ is archimedean, we set

$$
F_{v}^{0}=\{x \in \mathbb{R} \mid x \geq 0\}
$$

and

$$
\hat{F}_{v}=\{x \in \mathbb{R} \mid x \geq 1\}
$$

If $v$ is non-archimedean, we fix a uniformizer $\varpi$ of $F_{v}$, and set

$$
F_{v}^{0}=\left\{\varpi^{n} \mid n \in \mathbb{Z}\right\}
$$

and

We set

$$
\hat{F}_{v}=\left\{\varpi^{-n} \mid n \in \mathbb{N}\right\}
$$

$$
\mathrm{S}_{v}\left(F_{v}\right)^{0}=\left\{a \in \mathrm{S}_{v}\left(F_{v}\right) \mid \alpha(a) \in F_{v}^{0} \text { for each } \alpha \in \mathfrak{X}^{*}\left(\mathrm{~S}\left(F_{v}\right)\right)\right\}
$$

and

$$
\mathrm{S}_{v}\left(F_{v}\right)^{+}=\left\{a \in \mathrm{S}\left(F_{v}\right) \mid \alpha(a) \in \hat{F}_{v} \text { for each } \alpha \in \Phi^{+}\right\} .
$$

Similarly we may define $\mathrm{S}_{v}^{\prime}\left(F_{v}\right)^{+}$. We define constants $\kappa_{\alpha}$ for $\alpha \in \Delta\left(\mathrm{G}^{s p}, \mathrm{~T}^{s p}\right)$ by

$$
\sum_{\alpha>0, \alpha \in \Phi(\mathrm{G}, \mathrm{T})} \alpha=\sum_{\alpha \in \Delta(\mathrm{G}, \mathrm{T})} \kappa_{\alpha} \alpha .
$$

1.3. Integrality. By assumption, $G$ and $\mathrm{G}^{\prime}$ are of adjoint type and we can identify $\operatorname{Inn}\left(\mathrm{G}^{\prime}\right)=\mathrm{G}^{\prime}$. Then, for $g \in \mathrm{G}^{\prime}(E)$, we have

$$
c(\sigma) \cdot g=a_{\sigma} g a_{\sigma}^{-1}
$$

for a uniquely defined element $a_{\sigma} \in \mathrm{G}^{\prime}(E)$. For $w \in \operatorname{Val}(E)$ and $v \in \operatorname{Val}(F)$ with $w \mid v$, we have a restriction homomorphism

$$
\begin{array}{ccc}
\iota_{w}: \quad Z^{1}\left(\Gamma, \mathrm{G}^{\prime}(E)\right) & \rightarrow & Z^{1}\left(\Gamma_{w / v}, \mathrm{G}^{\prime}\left(E_{w}\right)\right), \\
c & \mapsto & c_{w} .
\end{array}
$$

The group $\mathrm{G}_{v}=\mathrm{G}_{F_{v}}$ is obtained from the corresponding quasi-split group $\mathrm{G}_{v}^{\prime}$ by twisting with $c_{w}$. With our choice of the set $S_{E}$ from Section 1.1, the local cocycle $c_{w}$ splits for $w \notin S_{E}$. Thus for $w \notin S_{E}, \sigma \in \Gamma_{w}$, we have

$$
c_{w}(\sigma)=a_{w} \cdot \sigma\left(a_{w}^{-1}\right)
$$

for some element $a_{w} \in \mathrm{G}^{\prime}\left(E_{w}\right)$. Identifying $\mathrm{G}^{\prime}(E)=\mathrm{G}(E)$ we may regard

- $\mathrm{G}^{\prime}(F)$ as the $\Gamma$-fixed points of $\mathrm{G}^{\prime}(E)$ for the standard action, and

- $\mathrm{G}(F)$ as the $\Gamma$-fixed points of $\mathrm{G}^{\prime}(E)$ for the twisted action.

Locally, we have a similar situation:

and further

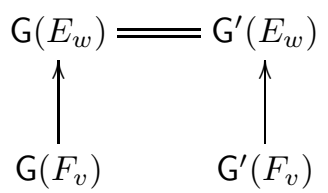

$$
a_{w}^{-1} \mathrm{G}\left(F_{v}\right) a_{w}=\mathrm{G}^{\prime}\left(F_{v}\right) .
$$

Recall that the global group $\mathrm{G}^{\prime}$ splits over $E$ and put

$$
\mathfrak{g}^{\prime}=\operatorname{Lie}\left(\mathrm{G}^{\prime}\right) \text { and } \mathfrak{g}_{E}^{\prime}=\mathfrak{g}^{\prime}(E) .
$$


Let $\mathrm{T}^{\prime} \subset \mathrm{G}^{\prime}$ be a maximal split torus defined over $E$. Let

$$
\mathfrak{g}^{s p}=\operatorname{Lie}\left(\mathrm{G}^{s p}\right), \mathfrak{g}_{\mathbb{Q}}^{s p}=\mathfrak{g}^{s p}(\mathbb{Q}) .
$$

Let $\Lambda_{\mathbb{Q}}$ be a Chevalley lattice in $\mathfrak{g}_{\mathbb{Q}}^{s p}$ adapted to $\mathrm{T}^{s p}$. Set

$$
\Lambda_{E}^{\prime}:=\Lambda_{\mathbb{Q}} \otimes_{\mathbb{Z}} \mathcal{O}_{E}
$$

and also set

$$
\Lambda_{w}^{\prime}:=\Lambda_{E}^{\prime} \otimes \mathfrak{o}_{E} \mathcal{O}_{w} .
$$

Then $\Lambda_{w}^{\prime}$ is a Chevalley lattice in $\mathfrak{g}_{w}^{\prime}=\mathfrak{g}^{\prime}\left(E_{w}\right)$. Moreover, $\Lambda_{w}^{\prime}$ is adapted to the torus $\mathrm{T}_{w}^{\prime}$ obtained from $\mathrm{T}^{\prime}$ by base extension to $E_{w}$. Let $\mathrm{K}_{w}$ be the stabilizer of $\Lambda_{w}$ in $\mathrm{G}\left(E_{w}\right)=\mathrm{G}^{\prime}\left(E_{w}\right)$. We have (see [23, Proposition 2.33)

$$
\mathrm{G}_{w}=\mathrm{K}_{w} \cdot \mathrm{B}\left(E_{w}\right)=\mathrm{B}\left(E_{w}\right) \cdot \mathrm{K}_{w} .
$$

Proposition 1.1. Let $v$ be a finite place of $F$ and $E_{w}$ an unramified extension of $F_{v}$. An element $a_{w} \in \mathrm{G}\left(E_{w}\right)=\mathrm{G}^{\prime}\left(E_{w}\right)$ satisfies

$$
a_{w} \sigma\left(a_{w}\right)^{-1} \in \mathrm{K}_{w}
$$

if and only if it is of the form

$$
a_{w}=k_{w} \cdot \gamma_{w}
$$

with $k_{w} \in \mathrm{K}_{w}$ and $\gamma_{w} \in \mathrm{G}^{\prime}\left(F_{v}\right)$.

We first recall the following

Theorem 1.2 (see 33, p. 292). Let $\mathrm{G}_{v}$ be a connected semi-simple group over $F_{v}$. Suppose that $\mathrm{G}_{v}$ is defined over $\mathfrak{o}_{v}$ and that as a group scheme over $\mathfrak{o}_{v}$, it has a connected smooth reduction modulo $\mathfrak{p}_{v}$. Let $E_{w}$ be a finite unramified extension of $F_{v}$. Then

$$
H^{1}\left(\Gamma_{w}, \mathrm{G}\left(\mathcal{O}_{w}\right)\right)=1 .
$$

Proof of Proposition 1.1. Consider the exact sequence of $\Gamma_{w}$-modules

$$
1 \rightarrow \mathrm{G}_{v}^{\prime}\left(\mathcal{O}_{w}\right) \rightarrow \mathrm{G}_{v}^{\prime}\left(E_{w}\right) \rightarrow \mathrm{G}_{v}^{\prime}\left(\mathcal{O}_{w}\right) \backslash \mathrm{G}_{v}^{\prime}\left(E_{w}\right) \rightarrow 1
$$

and the corresponding exact sequence in cohomology

$$
1 \rightarrow \mathrm{G}_{v}^{\prime}\left(\mathcal{O}_{v}\right) \rightarrow \mathrm{G}_{v}^{\prime}\left(F_{v}\right) \rightarrow\left(\mathrm{G}_{v}^{\prime}\left(\mathcal{O}_{w}\right) \backslash \mathrm{G}_{v}^{\prime}\left(E_{w}\right)\right)^{\Gamma_{w}} \rightarrow H^{1}\left(\Gamma, \mathrm{G}_{v}^{\prime}\left(\mathcal{O}_{w}\right)\right)=1,
$$

by Theorem 1.2 Consequently,

$$
\mathrm{G}_{v}^{\prime}\left(\mathcal{O}_{v}\right) \backslash \mathrm{G}_{v}^{\prime}\left(F_{v}\right)=\left(\mathrm{G}_{v}^{\prime}\left(\mathcal{O}_{w}\right) \backslash \mathrm{G}_{v}^{\prime}\left(E_{w}\right)\right)^{\Gamma_{w}},
$$

precisely the statement of Proposition 1.1.

1.4. Good primes. To apply Proposition 1.1 we will enlarge $S_{E}$, and $S_{F}$, so that for all $v \notin S_{F}$ one has:

- $\mathrm{G}^{\prime}$ has a group-scheme structure via the Chevalley lattice $\Lambda_{F}$;

- $\mathrm{G}^{\prime}$ has a connected smooth reduction modulo $\mathfrak{p}_{v}$;

- for all $\sigma \in \Gamma$

$$
c(\sigma) \in \mathrm{G}^{\prime}\left(\mathcal{O}_{w}\right)=\mathrm{K}_{w}^{\prime}
$$

- the local cocycle

$$
c_{w} \in Z^{1}\left(\Gamma_{w}, \mathrm{G}^{\prime}\left(E_{w}\right)\right)
$$

splits. 
Under these assumptions, for $\sigma \in \Gamma_{w}$ we may write

$$
c_{w}(\sigma)=a_{w} \cdot \sigma\left(a_{w}^{-1}\right)
$$

for some $a_{w} \in \mathrm{G}^{\prime}\left(E_{w}\right)$. Then, for $w \notin S$,

$$
a_{w} \cdot \sigma\left(a_{w}^{-1}\right) \in \mathrm{K}_{w}^{\prime} .
$$

By Proposition 1.1, we have $a_{w}=k_{w} \cdot \gamma_{w}$, for some $k_{w} \in \mathrm{K}_{w}^{\prime}, \gamma_{w} \in \mathrm{G}^{\prime}\left(F_{v}\right)$. Since $\sigma\left(\gamma_{w}\right)=\gamma_{w}$ for $\sigma \in \Gamma_{w}$, we may assume that

$$
c_{w}(\sigma)=a_{w} \cdot \sigma\left(a_{w}^{-1}\right)
$$

with $a_{w} \in \mathrm{K}_{w}^{\prime}$. Our main conclusion is

Corollary 1.3. There is a unique element $a_{w} \in \mathrm{K}_{w}^{\prime}$ such that

$$
a_{w}^{-1} \mathrm{G}\left(F_{v}\right) a_{w}=\mathrm{G}^{\prime}\left(F_{v}\right) .
$$

Proof. We only need to prove uniqueness. Suppose that we have another element $b \in \mathrm{G}\left(E_{w}\right)$ such that

$$
b^{-1} \mathrm{G}\left(F_{v}\right) b=\mathrm{G}^{\prime}\left(F_{v}\right) \text {. }
$$

Let $z=b a^{-1}$. Then

$$
z \mathrm{G}\left(F_{v}\right) z^{-1}=\mathrm{G}\left(F_{v}\right) .
$$

Next, let $\sigma \in \operatorname{Gal}\left(E_{w} / F_{v}\right)$. Then from (1.4) we have

$$
\tilde{\sigma}\left(z g z^{-1}\right)=z g z^{-1}
$$

for all $g \in \mathrm{G}\left(F_{v}\right)$. Hence, $z^{-1} \sigma(z)$ belongs to the centralizer of $\mathrm{G}\left(F_{v}\right)$ in $\mathrm{G}\left(\bar{F}_{v}\right)$. But then by a theorem of Rosenlicht, $\mathrm{G}\left(F_{v}\right)$ is Zariski dense in $\mathrm{G}\left(\bar{F}_{v}\right)$ (see [8, p. 11). Thus $z \in \mathrm{Z}\left(\bar{F}_{v}\right)$ (the center of $\mathrm{G}\left(\bar{F}_{v}\right)$ ). Since $\mathrm{G}$ is an adjoint group, we then have $z=1$ and $a=b$.

We set $\mathrm{K}_{v}^{\prime}=\mathrm{K}_{w}^{\prime} \cap \mathrm{G}^{\prime}\left(F_{v}\right)$. For $v \notin S_{F}$, this is a maximal compact subgroup in $\mathrm{G}^{\prime}\left(F_{v}\right)$. Put $\mathrm{K}_{v}=a_{w} \mathrm{~K}_{v}^{\prime} a_{w}^{-1}$. Then

Corollary 1.4 (Cartan Decomposition). For $v \notin S_{F}$, we have

$$
\mathrm{G}\left(F_{v}\right)=\mathrm{K}_{v} \mathrm{~S}\left(F_{v}\right)^{+} \mathrm{K}_{v} .
$$

Remark 1.5. If $v \in S_{F}$, then there is a maximal compact subgroup $\mathrm{K}_{v}$ of $\mathrm{G}\left(F_{v}\right)$ and a finite set $\Omega_{v} \subset \mathrm{G}\left(F_{v}\right)$ such that

$$
\mathrm{G}\left(F_{v}\right)=\mathrm{K}_{v} \mathrm{~S}\left(F_{v}\right)^{+} \Omega_{v} \mathrm{~K}_{v} .
$$

The set $\Omega_{v}$ is trivial if $v$ is archimedean, or if $\mathrm{G}\left(F_{v}\right)$ is split, or if $\mathrm{G}\left(F_{v}\right)$ is quasi-split and split over an unramified extension.

1.5. Galois cohomology of simply-connected groups. Here we recall basic facts about simply-connected semi-simple groups over the adeles.

Theorem 1.6 (Kneser). Let $\mathrm{H}$ be a simply-connected semi-simple group over $F$. Then, for $v \nmid \infty$,

$$
H^{1}\left(\Gamma_{v}, \mathrm{H}\left(\bar{F}_{v}\right)\right)=1,
$$

and the natural map

$$
H^{1}\left(\Gamma, \mathrm{H}^{s c}(F)\right) \rightarrow \prod_{v \mid \infty} H^{1}\left(\Gamma_{v}, H\left(\bar{F}_{v}\right)\right.
$$

is a bijection. 
It is useful to keep in mind the following:

Lemma 1.7 (Theorem 6.17 of [33]). Let $\mathrm{H}$ be a connected compact algebraic group over $\mathbb{R}$. Then

$$
H^{1}\left(\Gamma_{\mathbb{R}}, \mathrm{H}(\mathbb{C})\right)=\mathrm{A}^{\prime} / \mathrm{W}_{\mathrm{A}}
$$

where

$$
\mathrm{A}^{\prime}:=\left\{t \in \mathrm{A} \mid t^{2}=1\right\}
$$

$\mathrm{A} \subset \mathrm{H}$ is the maximal $\mathbb{R}$-torus and $\mathrm{W}_{\mathrm{A}}=\mathrm{N}_{\mathrm{H}}(\mathrm{A}) / \mathrm{A}$ is the corresponding Weyl group. Here, $\mathrm{N}_{\mathrm{H}}(\mathrm{A})$ is the normalizer of $\mathrm{A}$ in $\mathrm{H}$.

1.6. Norm maps. Let $\mathrm{T}$ be an algebraic torus over $F$, splitting over a normal extension $E / F$ with Galois group $\Gamma, v$ a place of $F, w \in \operatorname{Val}(E)$ a place over $v$, and $\Gamma_{w / v}$ the corresponding local Galois group. We have the following natural norm homomorphisms:

$$
\begin{array}{cclccl}
\mathrm{T}\left(E_{w}\right) & \stackrel{\mathrm{N}_{w / v}}{\longrightarrow} & \mathrm{T}\left(F_{v}\right), & \mathrm{T}\left(E_{w}\right) & \stackrel{\mathrm{N}_{v}}{\longrightarrow} & \mathrm{T}\left(F_{v}\right), \\
t_{w} & \mapsto & \prod_{\sigma \in \Gamma_{w / v}} \sigma\left(t_{w}\right), & t_{w} & \mapsto & \prod_{w \mid v} \mathrm{~N}_{w / v}\left(t_{w}\right), \\
\mathrm{T}(E) & \stackrel{\mathrm{N}_{E / F}}{\longrightarrow} & \mathrm{T}(F), & \mathrm{T}\left(\mathbb{A}_{E}\right) & \stackrel{\mathrm{N}_{\mathbb{A}_{E} / \mathbb{A}_{F}}}{\longrightarrow} & \mathrm{T}\left(\mathbb{A}_{F}\right), \\
t & \mapsto & \prod_{\sigma \in \Gamma} \sigma(t), & \left(t_{w}\right)_{w} & \mapsto & \left(\mathrm{N}_{v}\left(t_{w}\right)\right)_{v} .
\end{array}
$$

Lemma 1.8. For $t \in \mathrm{T}(E)$ one has $\mathrm{N}_{E / F}(t)=\mathrm{N}_{\mathbb{A}_{E} / \mathbb{A}_{F}}(t)$.

Proof. Let $w$ be a place of $E$ over $v$ and $\Gamma_{w} \subset \Gamma$ the fixer of $w$. For any $\sigma \in \Gamma$ we have $\sigma \Gamma_{w} \sigma^{-1}=\Gamma_{\sigma(w)}$ and $|x|_{w}=|\sigma(x)|_{\sigma(w)}$. For a fixed $w_{1}$ write

$$
\Gamma=\bigcup_{j=1}^{g} \sigma_{j} \Gamma_{w_{1}}=\bigcup_{j=1}^{g} \Gamma_{w_{1}} \sigma_{j}^{-1} .
$$

We have, for $t \in \mathrm{T}(E)$,

$$
\begin{aligned}
\mathrm{N}_{E / F}(t) & =\prod_{j=1}^{g} \prod_{\rho \in \Gamma_{w_{1}}} \rho \sigma_{j}^{-1}(t) \\
& =\prod_{j=1}^{g} \sigma_{j}^{-1} \prod_{\rho \in \Gamma_{w_{1}}} \sigma_{j} \rho \sigma_{j}^{-1}(t) \\
& =\prod_{j=1}^{g} \sigma_{j}^{-1} \prod_{\rho \in \Gamma_{w_{j}}} \rho(t) .
\end{aligned}
$$

Here $w_{j}=\sigma_{j} w_{1}$. We have the commutative diagram

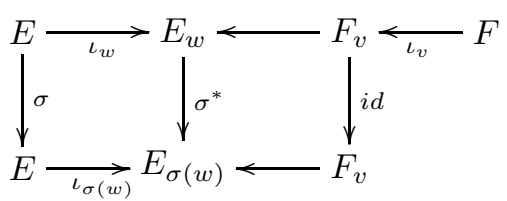

where $\iota_{v}, \iota_{w}$ are the canonical maps and $\sigma^{*}$ is the continuous extension of $\sigma$. 
For $a_{j}:=\prod_{\rho \in \Gamma_{w_{j}}} \rho(t)$ we have

$$
\begin{aligned}
\iota_{v}\left(\mathrm{~N}_{E / F}(t)\right) & =\iota_{w_{1}}\left(\mathrm{~N}_{E / F}(t)\right) \\
& =\prod_{j=1}^{g} \iota_{w_{1}} \sigma_{j}^{-1}\left(a_{j}\right) \\
& =\prod_{j=1}^{g}\left(\sigma_{j}^{*}\right)^{-1} \cdot \iota_{w_{j}}\left(a_{j}\right) .
\end{aligned}
$$

Next, for each $w$, let

$$
\Gamma_{w}^{*}:=\left\{\rho^{*} \mid \rho \in \Gamma_{w}\right\} .
$$

If $\rho \in \Gamma_{w_{j}}$, then $\rho\left(a_{j}\right)=a_{j}$. Thus

$$
\iota_{w_{j}}\left(a_{j}\right)=\iota_{w_{j}} \rho\left(a_{j}\right)=\rho^{*}\left(\iota_{w_{j}}\left(a_{j}\right)\right) .
$$

Thus $\iota_{w_{j}}(a)$ is fixed by $\Gamma_{w}^{*}$ and therefore belongs to $F_{v}$. But then

$$
\left(\sigma_{j}^{*}\right)^{-1}\left(\iota_{w_{j}}\left(a_{j}\right)\right)=\iota_{w_{j}}\left(a_{j}\right) .
$$

Thus

$$
\iota_{v}\left(\mathrm{~N}_{E / F}(t)\right)=\prod_{j=1}^{g} \iota_{w_{j}}\left(a_{j}\right)
$$

On the other hand,

$$
\begin{aligned}
\mathrm{N}_{\mathbb{A}_{E} / \mathbb{A}_{F}}(t) & =\prod_{j=1}^{g} \mathrm{~N}_{E_{w_{j}} / F_{v}}\left(\iota_{w_{j}}(t)\right) \\
& =\prod_{j=1}^{g} \prod_{\rho^{*} \in \Gamma_{w_{j}}^{*}} \rho^{*} \iota_{w_{j}}(t) \\
& =\prod_{j=1}^{g} \prod_{\rho \in \Gamma_{w_{j}}} \iota_{w_{j}}(\rho(t)) \\
& =\prod_{j=1}^{g} \iota_{w_{j}}\left(a_{j}\right) .
\end{aligned}
$$

This completes the proof.

\section{OnE-DimENSIONAL AUtOMORPHIC REPRESENTATIONS}

In this section we define a transfer associating to each one-dimensional automorphic representation $\chi$ of $G(\mathbb{A})$ a one-dimensional automorphic representation $\chi^{\prime}$ of $\mathrm{G}^{\prime}(\mathbb{A})$ in such a way that it respects local isomorphisms. The basic idea is to define a local transfer, and then use invariance under $\mathrm{G}(F)$ and weak approximation to extend the definition to the entire $G(\mathbb{A})$. We will also define certain Euler products that will later be used to regularize height integrals. 


\subsection{Automorphic characters for simply-connected groups.}

Proposition 2.1. Let $F$ be a number field and $\mathrm{H}$ a connected simply-connected semi-simple group over $F$. Let $\chi$ be a one-dimensional automorphic representation of $\mathrm{H}(\mathbb{A})$. Then $\chi=1$.

Proof. Let $E / F$ be a finite Galois extension splitting $\mathrm{H}$. There are infinitely many $v \in \operatorname{Val}(F)$ which split completely in $E$. For such $v$ the local group $\mathrm{H}\left(F_{v}\right)$ is split. Since $\mathbf{H}$ is simply-connected, $\mathbf{H}\left(F_{v}\right)$, as an abstract group, is its own derived group (see 9], esp. 3.3.5, p. A-16). Thus $\left.\chi\right|_{\mathrm{H}\left(F_{v}\right)}=1$, and also $\left.\chi\right|_{\mathrm{H}(F)}=1$. By strong approximation (see Theorem 7.12, p. 427 of 33$]), \mathrm{H}\left(F_{v}\right) \mathrm{H}(F)$ is dense in $\mathrm{H}(\mathbb{A})$. It follows that $\chi=1$.

2.2. The local transfer. Let $\mathrm{G}$ be as in Section 1.2 and

$$
j_{v}: \mathrm{G}^{s c}\left(F_{v}\right) \rightarrow \mathrm{G}\left(F_{v}\right)
$$

be the canonical homomorphism from its simply-connected covering. Let $v$ be a finite place of $F$ and $\hat{\mathrm{G}}_{v}$ the group of characters $\chi_{v}$ of $\mathrm{G}\left(F_{v}\right)$ which are trivial on $j_{v}\left(\mathrm{G}^{s c}\left(F_{v}\right)\right)$.

By Kneser's theorem, Theorem 1.6, $H^{1}\left(F_{v}, \mathrm{G}^{s c}\left(\bar{F}_{v}\right)\right)=1$. We have an exact sequence

$$
1 \rightarrow \mathrm{Z}\left(F_{v}\right) \rightarrow \mathrm{G}^{s c}\left(F_{v}\right) \stackrel{j_{v}}{\longrightarrow} \mathrm{G}\left(F_{v}\right) \stackrel{\delta_{v}}{\longrightarrow} H^{1}\left(F_{v}, \mathrm{Z}\left(\bar{F}_{v}\right)\right) \rightarrow 1
$$

and a similar sequence for $\mathrm{G}^{\prime}$. Here $\mathrm{Z}$ is the center of $\mathrm{G}^{s c}$. We may identify

$$
\hat{\mathrm{G}}_{v}=H^{1}\left(F_{v}, \mathrm{Z}\left(\bar{F}_{v}\right)\right)^{\vee}
$$

(the character group of $H^{1}\left(F_{v}, \mathrm{Z}\left(\bar{F}_{v}\right)\right)$. Recall that $\mathrm{G}$ is obtained from $\mathrm{G}^{\prime}$ by replacing the standard Galois action by a twisted action, via some representative of $H^{1}\left(\operatorname{Gal}(\bar{F} / F), \operatorname{Inn}\left(\mathrm{G}^{\prime}(\bar{F})\right)\right)$. Since inner automorphisms of $\mathrm{G}^{\prime}(\bar{F})$ fix $\mathrm{Z}(\bar{F})$, these two actions coincide on $\mathbf{Z}(F)$, and similarly for $\mathbf{Z}\left(\bar{F}_{v}\right)$. By (2.1) we then get, for non-archimedean $v$, a natural isomorphism

$$
\operatorname{tr}_{v}: \hat{\mathrm{G}}_{v} \rightarrow \hat{\mathrm{G}}_{v}^{\prime} .
$$

Remark 2.2. We observe that equation (2.1) implies that there is a number $n$, independent of $v$, such that every occurring character of $\mathrm{G}\left(F_{v}\right)$ satisfies $\chi^{n}=1$. This follows from a case-by-case analysis of $H^{1}\left(F_{v}, Z\left(\bar{F}_{v}\right)\right)$ as carried out in 33], Section 6.5. We will come back to this point in Section 8.8.

2.3. Weak approximation. We now apply weak approximation (Theorem 7.7, p. 415 of [33]):

Theorem 2.3. The group $\mathrm{G}(F)$ is dense in $\mathrm{G}_{\infty}:=\prod_{v \mid \infty} \mathrm{G}\left(F_{v}\right)$.

For $v \mid \infty$, the map $j_{v}: \mathrm{G}^{s c}\left(F_{v}\right) \rightarrow \mathrm{G}\left(F_{v}\right)$ is submersive. Write $\mathrm{G}_{\infty}^{*} \subset \mathrm{G}_{\infty}$ for the image of $\prod_{v \mid \infty} \mathrm{G}^{s c}\left(F_{v}\right)$ under $\prod_{v \mid \infty} j_{v}$; it is an open subgroup. Thus

$$
p r_{\infty}(\mathrm{G}(F)) \cdot \mathrm{G}_{\infty}^{*}=\mathrm{G}_{\infty}
$$

and

$$
\mathrm{G}(\mathbb{A})=\mathrm{G}(F) \cdot \mathrm{G}_{0} \cdot \mathrm{G}_{\infty}^{*} .
$$

Here $p r_{\infty}$ is the product of the projection maps $p r_{v}: \mathrm{G}(F) \rightarrow \mathrm{G}\left(F_{v}\right)$ for $v \mid \infty$. 
2.4. Global transfer. Let $\chi=\prod_{v} \chi_{v}$ be a one-dimensional automorphic representation of $\mathrm{G}(\mathbb{A})$ such that $\chi_{v}$ is trivial on $j_{v}\left(\mathrm{G}^{s c}\left(F_{v}\right)\right)$, for all $v \in \operatorname{Val}(F)$. In particular, $\chi_{\infty}:=\prod_{v \mid \infty} \chi_{v}$ is trivial on $\mathrm{G}_{\infty}^{*}$. Using (2.2) we define

$$
\chi_{0}^{\prime}=\prod_{v \nmid \infty} \operatorname{tr}_{v}\left(\chi_{v}\right) .
$$

We also have

$$
\mathrm{G}^{\prime}(\mathbb{A})=\mathrm{G}^{\prime}(F) \cdot \mathrm{G}_{0}^{\prime} \cdot\left(\mathrm{G}_{\infty}^{\prime}\right)^{*}
$$

We extend $\chi_{0}^{\prime}$ to a character $\chi^{\prime}$ of $\mathrm{G}^{\prime}(\mathbb{A})$ by setting $\chi^{\prime}=1$ on $\mathrm{G}^{\prime}(F) \cdot\left(\mathrm{G}_{\infty}^{\prime}\right)^{*}$. Then $\chi^{\prime}$ is well-defined since it is trivial on the intersection

$$
\mathrm{G}_{0}^{\prime} \cap \mathrm{G}^{\prime}(F) \cdot\left(\mathrm{G}_{\infty}^{\prime}\right)^{*} \text {. }
$$

Indeed, write $\mathrm{G}(F)^{*}:=\left\{\gamma \in \mathrm{G}(F) \mid p r_{\infty}(\gamma) \in \mathrm{G}_{\infty}^{*}\right\}$, and similarly for $\mathrm{G}^{\prime}$. Note that

$$
p r_{0}\left(\mathrm{G}(F)^{*}\right)=\mathrm{G}\left(\mathbb{A}_{f}\right) \cap \mathrm{G}(F) \cdot \mathrm{G}_{\infty}^{*},
$$

and similarly for $\mathrm{G}^{\prime}$. It suffices to check that

$$
\delta_{0}\left(p r_{0}\left(\mathrm{G}(F)^{*}\right)\right)=\delta_{0}^{\prime}\left(p r_{0}\left(\mathrm{G}^{\prime}(F)^{*}\right)\right),
$$

where $\delta_{0}:=\prod_{v \nmid \infty} \delta_{v}$ and similarly, $\delta_{0}^{\prime}=\prod_{v \nmid \infty} \delta_{v}^{\prime}$. In fact, the character $\chi$ is trivial on $\mathrm{G}(F) \cdot \mathrm{G}_{\infty}^{*}$.

Next, the sequence

$$
\mathrm{G}^{s c}\left(F_{v}\right) \stackrel{j_{v}}{\longrightarrow} \mathrm{G}\left(F_{v}\right) \stackrel{\delta_{v}}{\longrightarrow} H^{1}\left(F_{v}, \mathrm{Z}\left(\bar{F}_{v}\right)\right)
$$

is exact for all $v$. Set $\delta_{\infty}=\prod_{v \mid \infty} \delta_{v}$. Then in particular, $\delta_{\infty}\left(\mathrm{G}_{\infty}^{*}\right)=\{e\}$. We also set $\delta_{\mathbb{A}}=\delta_{0} \times \delta_{\infty}=\prod_{v} \delta_{v}$. Then (2.3) is equivalent to

$$
\delta_{\mathbb{A}}\left(\mathrm{G}(F)^{*}\right)=\delta_{\mathbb{A}}^{\prime}\left(\mathrm{G}^{\prime}(F)^{*}\right) .
$$

Let $p_{v}$ be the canonical map

$$
p_{v}: H^{1}(F, \mathrm{Z}(\bar{F})) \rightarrow H^{1}\left(F_{v}, \mathrm{Z}\left(\bar{F}_{v}\right)\right)
$$

and let

$$
p: H^{1}(F, \mathrm{Z}(\bar{F})) \rightarrow \prod_{v} H^{1}\left(F_{v}, \mathrm{Z}\left(\bar{F}_{v}\right)\right)
$$

be the corresponding global map. We have $p \circ \delta_{F}=\delta_{\mathbb{A}}$, where $\delta_{F}$ is the coboundary map $\mathrm{G}(F) \rightarrow H^{1}(F, \mathrm{Z}(\bar{F}))$.

Proposition 2.4. Let $c \in H^{1}(F, \mathrm{Z}(\bar{F}))$. Then $c \in \delta_{F}\left(\mathrm{G}(F)^{*}\right)$ if and only if

$$
p_{v}(c)=c_{v}=0 \text { for all } v \mid \infty .
$$

Proof. We have a diagram

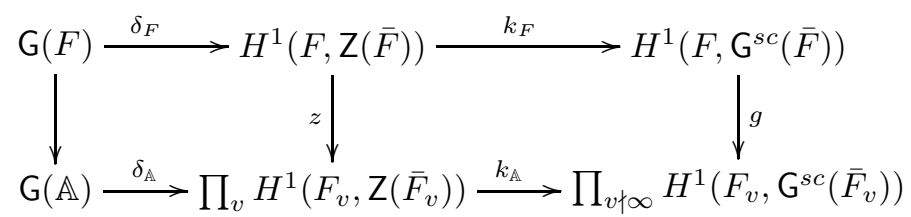

where $g$ is bijective (see Th. 6.6, p. 286 of 33 ), both squares are commutative and the top row is exact. Recall also that $H^{1}\left(F_{v}, G^{s c}\left(\bar{F}_{v}\right)\right)=\{e\}$, if $v$ is finite. 
Suppose that $c_{v}=z_{v}(c)=0$ for $v$ infinite, $c \in H^{1}(F, \mathrm{Z}(\bar{F}))$. Then $k_{\mathbb{A}}\left(\left(c_{v}\right)_{v}\right)=e$. Consequently, $k_{\mathbb{A}} \cdot z(c)=g \cdot k_{F}(c)=e$. Since $g$ is bijective, we have $k_{F}(c)=0$ and consequently

$$
c=\delta_{F}(\gamma)
$$

for some $\gamma \in \mathrm{G}(F)$. We prove that $\gamma \in \mathrm{G}(F)^{*}$. In fact, we have

$$
c_{v}=\delta_{v}\left(\gamma_{v}\right)=e, \text { for } v \mid \infty .
$$

But then, by the exactness of the sequence (2.4), $\gamma_{v} \in \operatorname{Im}\left(j_{v}\right)$, for any infinite $v$. Therefore, $\gamma \in \mathrm{G}_{\infty}^{*}$. The converse assertion also follows from the exactness of (2.4).

Corollary 2.5. As before, let

$$
\delta_{F}: \mathrm{G}(F) \rightarrow H^{1}(F, \mathrm{Z}(\bar{F}))
$$

and similarly let $\delta_{F}^{\prime}$ be the respective co-boundary maps. Then

$$
\delta_{F}\left(\mathrm{G}(F)^{*}\right)=\delta_{F}^{\prime}\left(\mathrm{G}^{\prime}(F)^{*}\right) .
$$

Proof. By the proposition, each of the sets $\delta_{F}\left(\mathrm{G}(F)^{*}\right)$ and $\delta_{F}^{\prime}\left(\mathrm{G}(F)^{*}\right)$ coincides with the set of $c \in H^{1}(F, \mathrm{Z}(\bar{F}))$ satisfying $c_{v}=e$ for $v \mid \infty$. Our assertion is now an immediate consequence.

2.5. Compatibility. The following lemma shows that the above definition of transfer is the correct one:

Lemma 2.6. Let $v \notin S_{F}$ and $w \in \operatorname{Val}(E)$ be such that $w \mid v$. Let $a_{w}$ be as in Corollary 1.3. Then for all $x \in \mathrm{G}\left(F_{v}\right)$ one has

$$
\chi_{v}^{\prime}\left(a_{w}^{-1} x a_{w}\right)=\chi_{v}(x)
$$

Proof. In this proof, we write $a$ for $a_{w}$. There is a character $\xi$ of the discrete group $H^{1}\left(F_{v}, \mathrm{Z}\left(\bar{F}_{v}\right)\right)$ such that

$$
\chi(x)=\xi\left(\delta_{v}(x)\right), x \in \mathrm{G}\left(F_{v}\right) \text { and } \chi^{\prime}(x)=\xi\left(\delta_{v}^{\prime}(x)\right), x \in \mathrm{G}^{\prime}\left(F_{v}\right) .
$$

It suffices to prove that $\delta_{v}^{\prime}\left(a^{-1} x a\right)=\delta_{v}(x)$. We calculate the two co-boundary maps, $\delta_{v}$ and $\delta_{v}^{\prime}$. Choose $\tilde{x} \in \mathrm{G}^{s c}\left(\bar{F}_{v}\right)$ such that $j_{v}(\tilde{x})=x$. Then

$$
\delta_{v}(x)=\tilde{x}^{-1} \sigma(\tilde{x})=\tilde{x}^{-1} c(\sigma) \circ \sigma^{\prime}(\tilde{x})=\tilde{x}^{-1} \tilde{a} \sigma^{\prime}(\tilde{a})^{-1} \sigma^{\prime}(\tilde{x}) \sigma^{\prime}(\tilde{a}) \tilde{a}^{-1} .
$$

Recall that, for $v \notin S_{F}$,

$$
c_{v}(\sigma)=a^{-1} \sigma^{\prime}(a)
$$

We calculate $\delta_{v}^{\prime}\left(a^{-1} x a\right)$, for $x \in \mathrm{G}\left(F_{v}\right)$. Choose $\tilde{a} \in \mathrm{G}^{s c}\left(\bar{F}_{v}\right)$ such that $\operatorname{Ad}(\tilde{a})=a$. We then have

$$
\delta_{v}^{\prime}\left(a^{-1} x a\right)=\left(\tilde{a}^{1} \tilde{x} \tilde{a}\right)^{-1} \sigma^{\prime}\left(\tilde{a}^{-1} \tilde{x} \tilde{a}\right)=\tilde{a}^{1} \tilde{x}^{-1} \tilde{a} \sigma^{\prime}\left(\tilde{a}^{-1}\right) \sigma^{\prime}(\tilde{x}) \sigma^{\prime}(\tilde{a}) .
$$

Since $\delta_{v}^{\prime}(x) \in \mathbf{Z}\left(\bar{F}_{v}\right)$, we have

$$
a \delta_{v}^{\prime}(x)=\delta_{v}^{\prime}(x) a
$$

or

$$
\tilde{a} \delta_{v}^{\prime}(x) \tilde{a}^{-1}=\delta_{v}^{\prime}(x)
$$

Thus

as claimed.

$$
\delta_{v}^{\prime}\left(a^{-1} x a\right)=\tilde{x}^{-1} \tilde{a} \sigma^{\prime}\left(\tilde{a}^{-1}\right) \sigma^{\prime}\left(\tilde{x}^{-1}\right) \sigma^{\prime}(\tilde{a}) \tilde{a}^{-1}=\delta_{v}(x),
$$


2.6. Continuity. Since $\mathbf{Z}$ is commutative, $H^{1}\left(F_{v}, \mathrm{Z}\left(\bar{F}_{v}\right)\right)$ is a (discrete) group and the co-boundary map

$$
\delta_{v}: \mathrm{G}\left(F_{v}\right) \rightarrow H^{1}\left(F_{v}, \mathrm{Z}\left(\bar{F}_{v}\right)\right)
$$

is a group homomorphism.

Lemma 2.7. The map $\delta_{v}$ is continuous.

Proof. The map

$$
j_{v}: \mathrm{G}^{s c}\left(F_{v}\right) \rightarrow \mathrm{G}\left(F_{v}\right)
$$

is a submersion of $v$-adic Lie groups (the differential of $j_{v}$ is the identity on the Lie algebra $\left.\operatorname{Lie}\left(\mathrm{G}^{s c}\left(F_{v}\right)\right)=\operatorname{Lie}\left(\mathrm{G}\left(F_{v}\right)\right)\right)$. Hence, $\operatorname{Im}\left(j_{v}\right)=\operatorname{Ker}\left(\delta_{v}\right)$ is an open subgroup of $\mathrm{G}\left(F_{v}\right)$, and the claim follows.

In any case, if $\chi_{v}: \mathrm{G}\left(F_{v}\right) \rightarrow \mathbb{C}^{*}$ is a homomorphism of groups which is trivial on the image of $j_{v}$, then $\chi_{v}$ is automatically continuous.

2.7. Automorphy. We have associated to each one-dimensional automorphic representation $\chi$ of $\mathrm{G}(\mathbb{A})$, trivial on $j\left(\mathrm{G}^{s c}(\mathbb{A})\right)$, a "formal" automorphic character

$$
\chi^{\prime}: \mathrm{G}^{\prime}(\mathbb{A}) \rightarrow \mathbb{C}^{\times}
$$

i.e., a homomorphism, trivial on $\mathrm{G}^{\prime}(F)$.

Theorem 2.8. The character $\chi^{\prime}$ is an automorphic character.

Proof. We need to show that $\chi^{\prime}$ is continuous. Since the map

$$
\delta_{v}^{\prime}: \mathrm{G}^{\prime}\left(F_{v}\right) \rightarrow H^{1}\left(F_{v}, \mathrm{Z}\left(\bar{F}_{v}\right)\right)
$$

is continuous, each $\chi_{v}^{\prime}$ is also continuous, for $v$ finite. By construction, $\chi_{\infty}^{\prime}=$ $\prod_{v \mid \infty} \chi_{v}^{\prime}$ is trivial on the open subgroup $\left(\mathrm{G}_{\infty}^{\prime}\right)^{*} \subset \mathrm{G}_{\infty}^{\prime}$. Thus, $\chi_{\infty}^{\prime}$ is continuous. The character $\chi_{v}$ is trivial on $\mathrm{K}_{v}$, for almost all $v$. We claim that $\chi^{\prime}$ is trivial on $\mathrm{K}_{v}^{\prime}$ for almost all $v$. This immediately follows from Lemma 2.6 and considerations of Section 1.4. We have already noted that $\chi_{v}^{\prime}$ is continuous for all $v$. It follows finally that $\chi^{\prime}$ is continuous.

2.8. Hecke characters. We now associate to $\chi^{\prime}$ a function $L\left(s, \chi^{\prime}\right)$, a product of Hecke $L$-functions. This function will later be used to regularize Fourier transforms of global height functions.

Suppose first that $\mathrm{G}_{F}^{\prime}=\mathrm{G}^{s p}$ is split. Let $\mathrm{T}^{s p} \subset \mathrm{B}^{s p} \subset \mathrm{G}^{s p}$ be a maximal split torus (over $F$ ), and a Borel subgroup containing T. Let $\Delta=\{\alpha\}$ be the associated set of simple roots and $\{\check{\alpha}\}$ the dual basis of the co-characters $\mathfrak{X}_{*}\left(\mathrm{~T}^{s p}\right)$. For $\lambda \in \mathbb{G}_{m}(\mathbb{A})$ and $\alpha \in \Delta$ define

$$
\xi_{\alpha}(\lambda)=\chi^{\prime}(\check{\alpha}(\lambda)) .
$$

This $\xi_{\alpha}$ is a character of $\mathbb{G}_{m}(\mathbb{A}) / \mathbb{G}_{m}(F)$ (Hecke-character). We define

$$
L\left(s, \chi^{\prime}\right)=\prod_{\alpha \in \Delta} L\left(s, \xi_{\alpha}\right) .
$$

In general, when $\mathrm{G}^{\prime}$ is quasi-split, and not necessarily split over $F$, we have

$$
\Phi\left(\mathrm{G}^{\prime}, \mathrm{T}^{\prime}\right) \subset \mathfrak{X}_{E}^{*}\left(\mathrm{~T}^{\prime}\right)=\mathfrak{X}^{*}\left(\mathrm{~T}^{\prime}\right) \text { and } \Phi\left(\mathrm{G}^{\prime}, \mathrm{S}^{\prime}\right) \subset \mathfrak{X}_{F}^{*}\left(\mathrm{~T}^{\prime}\right) .
$$

Then the restriction map

$$
r: \Phi\left(\mathrm{G}^{\prime}, \mathrm{T}^{\prime}\right) \rightarrow \Phi\left(\mathrm{G}^{\prime}, \mathrm{S}^{\prime}\right)
$$


is surjective, and

$$
r\left(\Delta\left(\mathrm{G}^{\prime}, \mathrm{T}^{\prime}\right)\right)=\Delta\left(\mathrm{G}^{\prime}, \mathrm{S}^{\prime}\right)
$$

The Galois group $\Gamma$ acts transitively on the fibers of

$$
r: \Delta\left(\mathrm{G}^{\prime}, \mathrm{T}^{\prime}\right) \rightarrow \Delta\left(\mathrm{G}^{\prime}, \mathrm{S}^{\prime}\right) .
$$

Fix a simple root $\alpha$ and let $\Gamma_{\alpha}$ be the stabilizer of $\alpha$ in $\Gamma$. Let $E_{\alpha}$ be the fixed field of $\Gamma_{\alpha}$. Since $\mathrm{G}$ is adjoint, for each $\alpha \in \Delta\left(\mathrm{G}^{\prime}, \mathrm{T}^{\prime}\right)$ we have an associated co-root $\check{\alpha}$ uniquely characterized by

$$
(\check{\alpha}, \beta)=\mathbf{1}_{\alpha \beta}
$$

where $($,$) is the natural \Gamma$-equivariant pairing

$$
\mathfrak{X}_{*}\left(\mathrm{~T}^{\prime}\right) \times \mathfrak{X}^{*}\left(\mathrm{~T}^{\prime}\right) \rightarrow \mathbb{Z}
$$

(and $\mathbf{1}$ is the delta function).

Since $\alpha$ is defined over $K:=E_{\alpha}$, so is $\check{\alpha}$. We then have a morphism

$$
\check{\alpha}: \mathbb{G}_{m} \rightarrow \mathrm{T}^{\prime},
$$

defined over $K$, and consequently a continuous homomorphism

$$
\check{\alpha}_{\mathbb{A}}: \mathbb{G}_{m}\left(\mathbb{A}_{K}\right) \rightarrow \mathrm{T}^{\prime}\left(\mathbb{A}_{K}\right) .
$$

Let $\phi_{\alpha}$ be the composite

$$
\phi_{\alpha}: \mathbb{G}_{m}\left(\mathbb{A}_{K}\right) \stackrel{\check{\alpha}_{\mathbb{A}}}{\longrightarrow} \mathrm{T}^{\prime}\left(\mathbb{A}_{K}\right) \stackrel{\mathrm{N}_{\mathbb{A}_{K} / \mathbb{A}_{F}}}{\longrightarrow} \mathrm{T}^{\prime}\left(\mathbb{A}_{F}\right) .
$$

By Section 1.6.

$$
\phi_{\alpha}: \mathbb{G}_{m}(K) \rightarrow \mathrm{T}^{\prime}(F) .
$$

Thus, if $\chi$ is a character of $\mathrm{T}^{\prime}\left(\mathbb{A}_{F}\right) / \mathrm{T}^{\prime}(F)$, then $\xi_{\alpha}=\chi \circ \phi_{\alpha}$ is an automorphic character of $\mathbb{G}_{m}$ over $K$, i.e., a Hecke character. Write $\xi_{\alpha}=\prod_{w \in \operatorname{Val}(K)} \xi_{\alpha, w}$.

Let $v$ be a place of $F, w$ a place of $K$ lying over $v$ and $u$ a place of $E$ lying over $w$. Let $F_{v} \subset K_{w} \subset E_{u}$ be the corresponding completions and $\iota_{w}, \iota_{w}, \iota_{u}$ the respective embeddings, with $\left.\iota_{u}\right|_{K}=\iota_{w},\left.\iota_{w}\right|_{F}=\iota_{v}$. Write $\mathfrak{X}_{E_{u}}^{*}\left(\mathrm{~T}^{\prime}\left(F_{v}\right)\right)=\mathfrak{X}^{*}\left(\mathrm{~T}^{\prime}\left(F_{v}\right)\right)$ and let

$$
\iota^{*}: \mathfrak{X}^{*}\left(\mathrm{~T}^{\prime}\right) \stackrel{\sim}{\longrightarrow} \mathfrak{X}^{*}\left(\mathrm{~T}^{\prime}\left(F_{v}\right)\right)
$$

be the isomorphism induced by $\iota_{u}$. As above, we have a restriction map

$$
r_{v}: \Phi\left(\mathrm{G}^{\prime}\left(F_{v}\right), \mathrm{T}^{\prime}\left(F_{v}\right)\right) \rightarrow \Phi\left(\mathrm{G}^{\prime}\left(F_{v}\right), \mathrm{S}_{v}^{\prime}\left(F_{v}\right)\right)
$$

mapping $\Delta\left(\mathrm{G}^{\prime}\left(F_{v}\right), \mathrm{T}^{\prime}\left(F_{v}\right)\right)$ to $\Delta\left(\mathrm{G}^{\prime}\left(F_{v}\right), \mathrm{S}_{v}^{\prime}\left(F_{v}\right)\right)$. The local Galois group $\Gamma_{u / v}$ acts transitively on the fibers of

$$
r_{v}: \Delta\left(\mathrm{G}^{\prime}\left(F_{v}\right), \mathrm{T}^{\prime}\left(F_{v}\right)\right) \rightarrow \Delta\left(\mathrm{G}^{\prime}\left(F_{v}\right), \mathrm{S}_{v}^{\prime}\left(F_{v}\right)\right)
$$

Next suppose that $K_{w} / F_{v}$ is unramified. Suppose also that $\xi_{\alpha, w}$ is unramified. Let

$$
L_{w}\left(s, \xi_{\alpha, w}\right)=\left(1-\xi_{\alpha, w}\left(\varpi_{w}\right) q_{w}^{-s}\right)^{-1}
$$

where $\varpi_{w}$ is a prime element for $K_{w}$. Let

$$
\alpha_{u}=\iota_{u}^{*}(\alpha), \vartheta_{v}=r_{v}\left(\alpha_{u}\right) \in \Delta\left(\mathrm{G}^{\prime}\left(F_{v}\right), \mathrm{S}_{v}^{\prime}\left(F_{v}\right)\right) .
$$

Let $\check{\vartheta}_{v} \in \mathfrak{X}_{*}\left(\mathrm{~S}_{v}^{\prime}\left(F_{v}\right)\right)$ be the associated co-character and let

$$
\begin{aligned}
\ell\left(\vartheta_{v}\right) & :=\#\left\{\beta \in \Delta\left(\mathrm{G}^{\prime}\left(F_{v}\right), \mathrm{T}^{\prime}\left(F_{v}\right)\right) \text { with } r_{u}(\beta)=\vartheta_{u}\right\} \\
& =\#\left\{\Gamma_{u / v}-\text { orbit of } \alpha_{u} \text { in } \Delta\left(\mathrm{G}^{\prime}\left(F_{v}\right), \mathrm{T}^{\prime}\left(F_{v}\right)\right)\right\} .
\end{aligned}
$$


Proposition 2.9. With the above notations, we have

$$
L_{w}\left(s, \xi_{\alpha, w}\right)=\left(1-\chi_{v}\left(\check{\vartheta}_{v}(\varpi)\right) \cdot q_{v}^{-\ell\left(\vartheta_{v}\right) s}\right)^{-1} .
$$

Proof. It suffices to prove

- $\ell\left(\vartheta_{u}\right)=\left[K_{w}: F_{v}\right]$

- $\xi_{\alpha, w}\left(\varpi_{w}\right)=\chi_{v}\left(\check{\vartheta}_{u}\left(\varpi_{w}\right)\right)$.

Let $\Gamma_{u / w}=\operatorname{Gal}\left(E_{u} / K_{w}\right)$. Then the fixer of $\alpha_{u}$ in $\Delta\left(\mathrm{G}^{\prime}\left(F_{v}\right), \mathrm{T}^{\prime}\left(F_{v}\right)\right)$ is $\Gamma_{u / w}$. Thus

$$
\ell\left(\vartheta_{u}\right)=\left[\Gamma_{u / v}: \Gamma_{u / w}\right]=\left[K_{w}: F_{v}\right] .
$$

For the the second assertion it suffices to prove that

$$
\mathrm{N}_{K_{w} / F_{v}}\left(\check{\alpha}_{w}(\lambda)\right)=\check{\vartheta}_{u}(\lambda), \lambda \in F_{v}^{\times} .
$$

The map $\iota_{u}$ induces an isomorphism

$$
\iota_{u}^{*}: \mathfrak{X}_{*}\left(\mathrm{~T}^{\prime}\right) \stackrel{\sim}{\longrightarrow} \mathfrak{X}_{*}\left(\mathrm{~T}^{\prime}\left(F_{v}\right)\right) .
$$

Then $\iota_{u}^{*}$ preserves the natural pairing of roots and co-characters. The co-character $\check{\alpha}_{w}$ is obtained from $\check{\alpha}$ by base extension to $K_{w}$ :

$$
\begin{gathered}
\check{\alpha}: \mathbb{G}_{m} / K \rightarrow \mathrm{T}^{\prime} / K, \\
\check{\alpha}_{w}: \mathbb{G}_{m} / K_{w} \rightarrow \mathrm{T}^{\prime} / K_{w} .
\end{gathered}
$$

We have for $\lambda \in F_{v}^{*}$

$$
\begin{aligned}
c & =\mathrm{N}_{K_{w} / F_{v}}\left(\check{\alpha}_{w}(\lambda)\right) \\
& =\prod_{\sigma \in \Gamma_{u / v}} \sigma\left(\check{\alpha}_{w}\left(\sigma^{-1}(\lambda)\right)\right) \\
& =\prod_{\sigma \in \Gamma_{u / v} / \Gamma_{u / w}} \sigma \circ\left(\check{\alpha}_{w}(\lambda)\right) .
\end{aligned}
$$

Here we regard $\check{\alpha}_{w} \in \mathfrak{X}_{*}\left(\boldsymbol{T}^{\prime}\left(F_{v}\right)\right)$ and $\sigma$ is the natural action of $\Gamma_{u / v}$ on $\mathfrak{X}_{*}\left(\mathbf{T}^{\prime}\left(F_{v}\right)\right)$. Since $c$ is fixed by $\Gamma_{u / v}$, we have $c \in \mathfrak{X}_{*}\left(\mathrm{~S}_{v}\left(F_{v}\right)\right)$. In fact, the morphism $\mathbb{G}_{m} \stackrel{c}{\longrightarrow}$ $\mathrm{T}^{\prime}\left(F_{v}\right)$ is defined over $F_{v}$. Let $\tilde{\mathrm{S}}\left(F_{v}\right)$ be the image of $c$ in $\mathrm{T}^{\prime}\left(F_{v}\right)$. Then $\tilde{\mathrm{S}}\left(F_{v}\right)$. $\mathrm{S}_{v}\left(F_{v}\right)=\mathrm{S}_{v}\left(F_{v}\right)$, since $\mathrm{S}_{v}^{\prime}$ is a maximal split $F_{v}$-torus in $\mathrm{T}^{\prime}\left(F_{v}\right)$. Thus $\tilde{\mathrm{S}}\left(F_{v}\right) \subset \mathrm{S}_{v}$ and we have $\mathbb{G}_{m} \stackrel{c}{\longrightarrow} \mathrm{S}_{v}^{\prime}$.

It will suffice to prove that $c=\check{\vartheta}_{u}$, as elements of $\mathfrak{X}_{*}\left(\mathrm{~S}_{v}^{\prime}\left(F_{v}\right)\right)$ :

$$
(c, \gamma)_{S_{v}^{\prime}}=\left(\check{\vartheta}_{u}, \gamma\right)_{S_{v}^{\prime}}
$$

for all $\gamma \in \Delta\left(\mathrm{G}^{\prime}\left(F_{v}\right), \mathrm{S}_{v}^{\prime}\left(F_{v}\right)\right)$. For this it suffices to prove that

- $\left(c, \vartheta_{u}\right)_{S_{v}^{\prime}}=1$;

- $(c, \gamma)_{\mathbf{S}_{v}^{\prime}}=0$ for all $\gamma \in \Delta\left(\mathrm{G}^{\prime}\left(F_{v}\right), \mathrm{S}_{v}^{\prime}\left(F_{v}\right)\right)$ with $\gamma \neq \vartheta_{u}$.

We have

$$
\left(c, \vartheta_{v}\right)_{\mathrm{S}_{v}^{\prime}}=\left(c, \alpha_{u}\right)_{\mathrm{T}^{\prime}\left(F_{v}\right)}=\sum_{\sigma \in \Gamma_{u / v} / \Gamma_{u / w}}\left(\sigma \circ \check{\alpha}_{w}, \alpha_{u}\right)_{\mathrm{T}^{\prime}\left(F_{v}\right)} .
$$

Next we have $\check{\alpha}_{w}=\check{\alpha}_{u}$ in $\mathfrak{X}_{*}\left(\mathbf{T}^{\prime}\left(F_{v}\right)\right)$. Here

$$
\check{\alpha}_{w}: \mathbb{G}_{m} / E_{u} \rightarrow \mathrm{T}^{\prime} / E_{u}
$$

is obtained by base extension from

$$
\check{\alpha}: \mathbb{G}_{m} / E \rightarrow \mathrm{T}^{\prime} / E,
$$


and

$$
\alpha_{u}: \mathrm{T}^{\prime} / E_{u} \rightarrow \mathbb{G}_{m} / E_{u}
$$

is obtained by base extension from

$$
\alpha: \mathrm{T}^{\prime} / E \rightarrow \mathbb{G}_{m} / E .
$$

Since extending the scalars preserves the natural pairing between roots and co-roots, we have the claim.

2.9. Infinite products. The next step is to express an infinite product of the form

$$
\prod_{v \in \operatorname{Val}(F)} \prod_{\vartheta \in \Delta\left(\mathrm{G}^{\prime}\left(F_{v}\right), \mathrm{S}_{v}^{\prime}\left(F_{v}\right)\right)}\left(1-\chi_{v}\left(\check{\vartheta}\left(\varpi_{w}\right)\right) q_{v}^{-\ell_{v}(\vartheta) s_{\vartheta}}\right)^{-1}
$$

in terms of Hecke $L$-functions; this will be important in the regularization of height integrals. The above expression, as written, has an infinite number of complex variables. In practice, the number of variables is finite, since the complex numbers $s_{\vartheta}$ are Galois invariant in the following sense.

Consider the commutative diagram

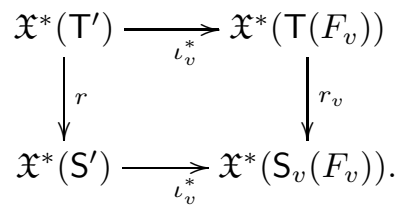

Let $\alpha \in \Delta\left(\mathrm{G}^{\prime}, \mathrm{T}^{\prime}\right)$ and let $\mathfrak{O}=\Gamma \cdot \alpha$ be the orbit of $\alpha$ (for the action of $\Gamma$ on $\left.\Delta\left(\mathrm{G}^{\prime}, \mathrm{T}^{\prime}\right)\right)$. We further set $\mathfrak{O}_{v}=r_{v}\left(\iota_{u}^{*}(\mathfrak{O})\right) \subset \Delta\left(\mathrm{G}^{\prime}\left(F_{v}\right), \mathrm{S}_{v}^{\prime}\left(F_{v}\right)\right)$. For each $\vartheta \in \mathfrak{O}_{v}$ choose a $\beta \in \Delta\left(\mathrm{G}^{\prime}, \mathrm{T}^{\prime}\right)$ so that $r_{u}\left(\iota_{u}^{*}(\beta)\right)=\vartheta$. We now require that $s_{\vartheta}$ depend only on the Galois orbit of $\beta$ in $\Delta\left(G^{\prime}, T^{\prime}\right)$, i.e., only on $\mathfrak{O}$; we will denote the common value of all such $s_{\vartheta}$ by $s_{\mathfrak{O}}$. With the assumption, the number of genuine complex parameters appearing in (2.7) is the number of distinct Galois orbits $\mathfrak{O}$ in $\Delta\left(G^{\prime}, T^{\prime}\right)$.

Fix an orbit $\mathfrak{O}$. For any $\beta \in \mathfrak{O}$, we have a field $E_{\beta}$ over which $\beta$ is defined. We have already described how to associate to $\beta$ a Hecke character $\xi_{\beta}(\chi)$ of $E_{\beta}$. The Hecke $L$-function $L\left(s, \xi_{\beta}(\chi)\right)$ depends only on the Galois orbit $\mathfrak{O}$, and not on the particular $\beta$. For this reason, we denote the $L$-function $L\left(s, \xi_{\beta}(\chi)\right)$ by $L\left(s, \xi_{\mathfrak{O}}(\chi)\right)$. An argument similar to the proof of Theorem 4.1.3 of [5] leads to the following proposition:

Proposition 2.10. We have

$$
\prod_{v \in \operatorname{Val}(F)} \prod_{\vartheta \in \Delta\left(\mathrm{G}^{\prime}\left(F_{v}\right), \mathrm{S}_{v}^{\prime}\left(F_{v}\right)\right)}\left(1-\chi_{v}\left(\check{\vartheta}\left(\varpi_{w}\right)\right) q_{v}^{-\ell_{v}(\vartheta) s_{\vartheta}}\right)=\prod_{\mathfrak{O}} L\left(s_{\mathfrak{O}}, \xi_{\mathfrak{O}}(\chi)\right),
$$

where the latter product is over all Galois orbits in $\Delta\left(\mathrm{G}^{\prime}, \mathrm{T}^{\prime}\right)$.

\section{Eisenstein SERIES AND SPECTRAL THEORY}

3.1. Basic spectral theory. Let B be a minimal parabolic subgroup of G. We will work only with standard parabolic subgroups and up to association. A typical parabolic subgroup is denoted by $P$. We denote the Levi factor of $P$ by $M_{P}$, its unipotent radical by $N_{P}$, and the split component of the center of $M_{P}$ by $A_{P}$. We let $\mathfrak{X}^{*}\left(M_{P}\right)_{\mathbb{Q}}$ be the group of characters of $M_{P}$ defined over $\mathbb{Q}$, and we set 
$\mathfrak{a}_{\mathrm{P}}=\operatorname{Hom}\left(\mathfrak{X}^{*}\left(\mathrm{M}_{\mathrm{P}}\right)_{\mathbb{Q}}, \mathbb{R}\right)$. Clearly, $\mathfrak{a}_{\mathrm{P}}^{*}=\mathfrak{X}^{*}\left(\mathrm{M}_{\mathrm{P}}\right)_{\mathbb{Q}} \otimes \mathbb{R}$. We denote by $\Delta_{\mathrm{P}}$ the set of the simple roots of $(P, A)$. We usually drop the subscript $P$.

For $m=\left(m_{v}\right)_{v} \in \mathrm{M}(\mathbb{A})$ define a vector $H_{\mathrm{M}}(m) \in \mathfrak{a}_{\mathrm{P}}$ by

$$
e^{\left\langle H_{\mathrm{M}}(m), \chi\right\rangle}=|\chi(m)|=\prod_{v}\left|\chi\left(m_{v}\right)\right|_{v}
$$

for all $\chi \in \mathfrak{X}^{*}(\mathrm{M})_{\mathbb{Q}}$. This is a homomorphism

$$
\mathrm{M}(\mathbb{A}) \longrightarrow \mathfrak{a}_{\mathrm{P}}
$$

We let $M(\mathbb{A})^{1}$ be the kernel. Then

$$
\mathrm{M}(\mathbb{A})=\mathrm{M}(\mathbb{A})^{1} \times \mathrm{A}(\mathbb{R})^{0}
$$

By Iwasawa decomposition, any $x \in \mathrm{G}(\mathbb{A})$ can be written as nmak with $n \in$ $\mathrm{N}(\mathbb{A}), m \in \mathrm{M}(\mathbb{A})^{1}, a \in \mathrm{A}(\mathbb{R})^{0}, k \in \mathrm{K}$. Set $H_{\mathrm{P}}(x):=H_{\mathrm{M}}(a) \in \mathfrak{a}_{\mathrm{P}}$. Denote the restricted Weyl group of $(G, A)$ by $W$. The group $W$ acts on $\mathfrak{a}_{B}$ and $\mathfrak{a}_{\mathrm{B}}^{*}$. For any $\mathbf{s} \in \mathbf{W}$, fix a representative $w_{\mathrm{s}}$ in the intersection of $\mathrm{G}(\mathbb{Q})$ with the normalizer of $A_{B}$. If $P_{1}, P_{2}$ are parabolic subgroups, we let $W\left(\mathfrak{a}_{1}, \mathfrak{a}_{2}\right)$ be the set of distinct isomorphisms $\mathfrak{a}_{1} \rightarrow \mathfrak{a}_{2}$ obtained by restricting elements of $W$ to $\mathfrak{a}_{1}$. The groups $P_{1}, P_{2}$ are called associated if $W\left(\mathfrak{a}_{1}, \mathfrak{a}_{2}\right)$ is not empty. We usually think of $W\left(\mathfrak{a}_{1}, \mathfrak{a}_{2}\right)$ as a subset of $\mathrm{W}$. We let $n(\mathrm{~A})$ be the number of chambers in $\mathfrak{a}$.

Set

$$
\mathfrak{a}_{\mathrm{P}}^{+}=\left\{H \in \mathfrak{a}_{\mathrm{P}} ; \alpha(H)>0 \text { for all } \alpha \in \Delta_{\mathrm{P}}\right\}
$$

and

$$
\left(\mathfrak{a}_{\mathrm{P}}^{*}\right)^{+}=\left\{\Lambda \in \mathfrak{a}_{\mathrm{P}}^{*} ; \Lambda(\check{\alpha})>0 \text { for all } \alpha \in \Delta_{\mathrm{P}}\right\} .
$$

There is a vector $\rho_{\mathrm{P}} \in\left(\mathfrak{a}_{\mathrm{P}}^{*}\right)^{+}$such that

$$
\delta_{\mathrm{P}}(p)=\left|\operatorname{det}\left(\left.A d p\right|_{\mathfrak{n}_{\mathrm{P}}(\mathbb{A})}\right)\right|=e^{2 \rho_{\mathrm{P}}\left(H_{\mathrm{P}}(p)\right)}
$$

for all $p \in \mathrm{P}(\mathbb{A})$. We take the normalization of Haar measures to be as in [1.

Let $L_{\text {cusp }}^{2}\left(M(\mathbb{Q}) \backslash M(\mathbb{A})^{1}\right)$ be the space of functions $\phi$ in $L^{2}\left(M(\mathbb{Q}) \backslash M(\mathbb{A})^{1}\right)$ such that for any parabolic $\mathrm{P}_{1} \nsubseteq \mathrm{P}$ we have

$$
\int_{\mathrm{N}_{1}(\mathbb{Q}) \cap \mathrm{M}(\mathbb{Q}) \backslash \mathrm{N}_{1}(\mathbb{A}) \cap \mathrm{M}(\mathbb{A})} \phi(n m) d n=0
$$

for almost all $m$. It is known that

$$
\mathrm{L}_{\text {cusp }}^{2}\left(\mathrm{M}(\mathbb{Q}) \backslash \mathrm{M}(\mathbb{A})^{1}\right)=\bigoplus_{\varrho} V_{\varrho}
$$

where $V_{\varrho}$ is the $\varrho$-isotypic component of $\varrho$ consisting of finitely many copies of $\varrho$ (possibly zero). The pairs $(\mathrm{M}, \varrho)$ and $\left(\mathrm{M}^{\prime}, \varrho^{\prime}\right)$ are considered equivalent if there is an $\mathbf{s} \in \mathrm{W}\left(\mathfrak{a}, \mathfrak{a}^{\prime}\right)$ such that the representation

$$
(\mathrm{s} \varrho)\left(m^{\prime}\right)=\varrho\left(w_{\mathrm{s}}^{-1} m^{\prime} w_{\mathrm{s}}\right) \quad\left(m^{\prime} \in \mathrm{M}^{\prime}(\mathbb{A})^{1}\right)
$$

is unitarily equivalent to $\varrho^{\prime}$. Let $\mathfrak{X}$ be the set of equivalence classes of such pairs. For any $\chi \in \mathfrak{X}$ we have a class $\mathcal{P}_{\chi}$ of associated parabolic subgroups. If $\mathrm{P}$ is any parabolic and $\chi \in \mathfrak{X}$, set

$$
\mathrm{L}_{\text {cusp }}^{2}\left(\mathrm{M}(\mathbb{Q}) \backslash \mathrm{M}(\mathbb{A})^{1}\right)_{\chi}=\bigoplus_{(\varrho:(\mathrm{M}, \varrho) \in \chi)} V_{\varrho} .
$$

This is a closed subspace of $\mathrm{L}_{\text {cusp }}^{2}\left(\mathrm{M}(\mathbb{Q}) \backslash \mathrm{M}(\mathbb{A})^{1}\right)$, and empty if $\mathrm{P} \notin \mathcal{P}_{\chi}$. 
Fix $\mathrm{P}$ and $\chi \in \mathfrak{X}$. Suppose there is a $\mathrm{P}_{1} \in \mathcal{P}_{\chi}$ such that $\mathrm{P}_{1} \subset \mathrm{P}$. Let $\psi$ be a smooth function on $\mathrm{N}_{1}(\mathbb{A}) \mathrm{M}_{1}(\mathbb{Q}) \backslash \mathrm{G}(\mathbb{A})$ such that

$$
\Psi_{a}(m, k)=\psi(a m k) \quad\left(k \in \mathrm{K}, m \in \mathrm{M}_{1}(\mathbb{Q}) \backslash \mathrm{M}_{1}(\mathbb{A}), a \in \mathrm{A}_{1}(\mathbb{Q}) \backslash \mathrm{A}_{1}(\mathbb{A})\right)
$$

vanishes outside a compact subset of $A_{1}(\mathbb{Q}) \backslash A_{1}(\mathbb{A})$, transforms under $K_{\mathbb{R}}$ according to an irreducible representation, and as a function of $m$ it belongs to $\mathrm{L}_{\text {cusp }}^{2}\left(\mathrm{M}(\mathbb{Q}) \backslash \mathrm{M}(\mathbb{A})^{1}\right)$. Then the function

$$
\hat{\psi}^{\mathrm{M}}(m)=\sum_{\gamma \in \mathrm{P}_{1}(\mathbb{Q}) \cap \mathrm{M}(\mathbb{Q}) \backslash \mathrm{M}(\mathbb{Q})} \psi(\gamma m) \quad\left(m \in \mathrm{M}(\mathbb{Q}) \backslash \mathrm{M}(\mathbb{A})^{1}\right)
$$

is square-integrable on $M(\mathbb{Q}) \backslash M(\mathbb{A})^{1}$. Define $L^{2}\left(M(\mathbb{Q}) \backslash M(\mathbb{A})^{1}\right)_{\chi}$ to be the span of all such $\hat{\psi}^{\mathrm{M}}$. If no such $\mathrm{P}_{1}$ exist, latter space is the zero space.

By a result of Langlands,

$$
L^{2}\left(M(\mathbb{Q}) \backslash M(\mathbb{A})^{1}\right)=\bigoplus_{\chi} L^{2}\left(M(\mathbb{Q}) \backslash M(\mathbb{A})^{1}\right)_{\chi} .
$$

For any $\mathrm{P}$, let $\Pi(\mathrm{M})$ denote the set of equivalence classes of irreducible unitary representations of $\mathrm{M}(\mathbb{A})$. For $\zeta \in \mathfrak{a}_{\mathbb{C}}^{*}$ and $\pi \in \Pi(\mathrm{M})$ let $\pi_{\zeta}$ be the product of $\pi$ with the quasi-character

$$
x \mapsto e^{\zeta H_{\mathrm{P}}(x)} \quad(x \in \mathrm{G}(\mathbb{A})) .
$$

If $\zeta \in i \mathfrak{a}^{*}, \pi_{\zeta}$ is again unitary. This means that $\Pi(\mathrm{M})$ is a differentiable manifold which carries an action of $i \mathfrak{a}^{*}$. We use this action to define a measure $d \pi$ on $\Pi(\mathrm{M})$.

For $\pi \in \Pi(\mathrm{M})$ we let $\mathcal{H}_{\mathrm{P}}^{0}(\pi)$ be the space of smooth functions

$$
\phi: \mathrm{N}(\mathbb{A}) \mathrm{M}(\mathbb{Q}) \backslash \mathrm{G}(\mathbb{A}) \rightarrow \mathbb{C}
$$

satisfying

(1) $\phi$ is right $\mathrm{K}$-finite;

(2) for every $x \in \mathrm{G}(\mathbb{A})$ the function

$$
m \mapsto \phi(m x) \quad(m \in \mathrm{M}(\mathbb{A}))
$$

is a matrix coefficient of $\pi$;

(3) $\|\phi\|^{2}=\int_{\mathrm{K}} \int_{\mathrm{M}(\mathbb{Q}) \backslash \mathrm{M}(\mathbb{A})^{1}}|\phi(m k)|^{2} d m d k<+\infty$.

Let $\mathcal{H}_{\mathrm{P}}(\pi)$ be the completion. If $\phi \in \mathcal{H}_{\mathrm{P}}(\pi)$ and $\zeta \in \mathfrak{a}_{\mathbb{C}}^{*}$ set

$$
\phi_{\zeta}(x)=\phi(x) e^{\zeta\left(H_{\mathrm{P}}(x)\right)} \quad(x \in \mathrm{G}(\mathbb{A}))
$$

and

$$
\left(I_{\mathrm{P}}\left(\pi_{\zeta}, y\right) \phi_{\zeta}\right)(x)=\phi_{\zeta}(x y) \delta_{\mathrm{P}}(x y)^{\frac{1}{2}} \delta_{\mathrm{P}}(x)^{-\frac{1}{2}} .
$$

Then $I_{\mathrm{P}}\left(\pi_{\zeta}\right)$ is a unitary representation if $\zeta \in i \mathfrak{a}^{*}$.

Given $\chi \in \mathfrak{X}$, let $\mathcal{H}_{\mathrm{P}}(\pi)_{\chi}$ be the closed subspace of $\mathcal{H}_{\mathrm{P}}(\pi)$ consisting of those $\phi$ such that for all $x$ the function $m \mapsto \phi(m x)$ belongs to $\mathrm{L}^{2}\left(\mathrm{M}(\mathbb{Q}) \backslash \mathrm{M}(\mathbb{A})^{1}\right)_{\chi}$. Then

$$
\mathcal{H}_{\mathrm{P}}(\pi)=\bigoplus_{\chi} \mathcal{H}_{\mathrm{P}}(\pi)_{\chi}
$$

Let $\mathrm{K}_{0}$ be an open-compact subgroup of $\mathrm{G}\left(\mathbb{A}_{f}\right)$ and $W$ an equivalence class of irreducible representations of $\mathrm{K}_{\mathbb{R}}$. Let $\mathcal{H}_{\mathrm{P}}(\pi)_{\chi}, \mathrm{K}_{0}$ be the subspace of functions in $\mathcal{H}_{\mathrm{P}}(\pi)_{\chi}$ which are invariant under $\mathrm{K}_{0} \cap \mathrm{K}$. Also let $\mathcal{H}_{\mathrm{P}}(\pi)_{\chi}, \mathrm{K}_{0}, W$ be the space of those functions in $\mathcal{H}_{\mathrm{P}}(\pi)_{\chi}, \mathrm{K}_{0}$ which transform under $\mathrm{K}_{\mathbb{R}}$ according to $W$. It is a theorem of Langlands that each of the spaces $\mathcal{H}_{\mathrm{P}}(\pi)_{\chi}, \mathrm{K}_{0}, W$ is finite-dimensional. 
We fix an orthonormal basis $\mathcal{B}(\pi)_{\chi}$ for $\mathcal{H}_{\mathrm{P}}(\pi)_{\chi}$, for each $\pi$ and each $X$, such that for all $\zeta \in i \mathfrak{a}^{*}$ we have

$$
\mathcal{B}\left(\pi_{\zeta}\right)_{\chi}=\left\{\phi_{\zeta}: \phi \in \mathcal{B}_{\mathrm{P}}(\pi)_{\chi}\right\}
$$

and such that every $\phi \in \mathcal{B}_{\mathrm{P}}(\pi)_{\chi}$ belongs to one of the spaces $\mathcal{H}_{\mathrm{P}}(\pi)_{\chi}, \mathrm{K}_{0}, W$.

Suppose that $\pi \in \Pi(\mathrm{M}), \phi \in \mathcal{H}_{\mathrm{P}}^{0}(\pi), \zeta \in \mathfrak{a}_{\mathbb{C}}^{*}$. For $\Re(\zeta) \in \rho_{\mathrm{P}}+\left(\mathfrak{a}^{*}\right)^{+}$we set

$$
E(x, \phi, \zeta)=\sum_{\gamma \in \mathrm{P}(\mathbb{Q}) \backslash \mathrm{G}(\mathbb{Q})} \phi_{\zeta}(\gamma x) \delta_{\mathrm{P}}(\gamma x)^{\frac{1}{2}} .
$$

For $\mathrm{s} \in \mathrm{W}\left(\mathfrak{a}, \mathfrak{a}^{\prime}\right)$, we define the global intertwining operator

$$
\begin{aligned}
& \left(M(\mathrm{~s}, \pi, \zeta) \phi_{\zeta}\right)(x) \\
& \quad=\int_{\mathbb{N}^{\prime}(\mathbb{A}) \cap w_{\mathrm{s}} \mathrm{N}(\mathbb{A}) w_{\mathrm{s}}^{-1} \backslash \mathrm{N}^{\prime}(\mathbb{A})} \phi_{\zeta}\left(w_{\mathrm{s}}^{-1} n x\right) \delta_{\mathrm{P}}\left(w_{\mathrm{s}}^{-1} n x\right)^{\frac{1}{2}} \delta_{\mathrm{P}}(x)^{-\frac{1}{2}} d n .
\end{aligned}
$$

Both $E(x, \phi, \zeta)$ and $M\left(\mathrm{~s}, \pi_{\zeta}\right) \phi_{\zeta}$ can be analytically continued to meromorphic functions in $\zeta$ to $\mathfrak{a}_{\mathbb{C}}^{*}$. For $\zeta \in i \mathfrak{a}^{*}, E(x, \phi, \zeta)$ is a smooth function of $x$ and $M\left(\mathrm{~s}, \pi_{\zeta}\right)$ is unitary from $\mathcal{H}_{\mathrm{P}}\left(\pi_{\zeta}\right)$ to $\mathcal{H}_{\mathrm{P}^{\prime}}\left(\mathrm{s} \pi_{\zeta}\right)$.

3.2. Spectral expansion. Let $f$ be a function on $G(\mathbb{Q}) \backslash G(\mathbb{A})$. By the spectral expansion of $f$ we mean

$$
\begin{array}{rl}
S(f, x)=\sum_{\chi \in \mathfrak{X}} \sum_{\mathrm{P}} & n(\mathrm{~A})^{-1} \\
& \times \int_{\Pi(\mathrm{M})} \sum_{\phi \in \mathcal{B}_{\mathrm{P}}(\pi)_{\chi}} E(x, \phi)\left(\int_{\mathrm{G}(\mathbb{Q}) \backslash \mathrm{G}(\mathbb{A})} \overline{E(y, \phi)} f(y) d y\right) d \pi .
\end{array}
$$

For $M=G$, the integrals are interpreted appropriately to give a discrete sum. It is an interesting problem to determine under which conditions on the function $f$ we have $f(x)=S(f, x)$. Also, for the application we have in mind, we need to know that the identity $f=S_{f}$ holds as an identity not just of $\mathrm{L}^{2}$-functions, but of continuous functions. In order to show that for a given $f$ the spectral identity holds, we just need to prove that the right hand side of (3.22) is uniformly convergent on compact sets. This would suffice since for all pseudo-Eisenstein series $\theta_{\phi}$ (II.1.10 of 27]) we have

$$
\left(S(f), \theta_{\phi}\right)=\left(f, S\left(\theta_{\phi}\right)\right),
$$

and since pseudo-Eisenstein series are rapidly decreasing (Proposition II.1.10 of [27]), we have $\theta_{\phi}=S\left(\theta_{\phi}\right)$ by the spectral decomposition [1]. Consequently

$$
\left(f-S(f), \theta_{\phi}\right)=0
$$

for all pseudo-Eisenstein series $\theta_{\phi}$. Then by the density theorem (Theorem II.1.12 of [27]) we get the required identity. In our applications, we use a slightly different re-arrangement of the terms of $S(f)$ as suggested by the definition of $I(S, f, x, y)$ on page 930 of $[1$.

Let $f$ be a smooth function on $G(\mathbb{A})$, and suppose that $f$ is right invariant under a compact-open subgroup $\mathrm{K}$ of $\mathrm{G}\left(\mathbb{A}_{f}\right)$. Define a function on $\mathrm{G}(\mathbb{Q}) \backslash \mathrm{G}(\mathbb{A})$ by

$$
F(g):=\sum_{\gamma \in \mathrm{G}(\mathbb{Q})} f(\gamma g) .
$$


Suppose that $f$ is such that the function $F$ is convergent for all $g$, is smooth and is bounded. By Lemma 4.1 of [1], given $m$, there is an $n$ and functions $f_{1} \in$ $C_{c}^{m}(\mathrm{G}(\mathbb{R}))^{\mathrm{K}_{\mathbb{R}}}$ and $f_{2} \in C_{c}^{\infty}(\mathrm{G}(\mathbb{R}))^{\mathrm{K}_{\mathbb{R}}}$ such that $\Delta^{n} * f_{1}+f_{2}$ is the delta distribution at the identity of $\mathrm{G}(\mathbb{R})$. Here $\Delta$ is an appropriate element of the enveloping algebra chosen as in the proof of Lemma 4.1 of [1]. This implies that after taking the finite places into account, we can find functions $\eta_{1}, \eta_{2}$ of compact support on $G(\mathbb{A})$, with appropriate differentiability at the archimedean place, such that

$$
R\left(\Delta^{n} * \eta_{1}+\eta_{2}\right) F=F,
$$

where $R$ is the right regular convolution action. We get

$$
R\left(\eta_{1}\right) \Delta^{n} F+R\left(\eta_{2}\right) F=F .
$$

Consider the following modified spectral expansion:

$$
\begin{array}{rl}
S^{\prime}(F, x)=\sum_{\chi \in \mathfrak{X}} \sum_{\mathrm{P}} & n(\mathrm{~A})^{-1} \\
& \times \int_{\Pi(\mathrm{M})} \int_{\mathrm{G}(\mathbb{Q}) \backslash \mathrm{G}(\mathbb{A})}\left(\sum_{\phi \in \mathcal{B}_{\mathrm{P}}(\pi)_{\chi}} E(x, \phi) \overline{E(y, \phi)}\right) F(y) d y d \pi .
\end{array}
$$

We get

$$
\begin{array}{rl}
S^{\prime}(F, x)=\sum_{\chi \in \mathfrak{X}} \sum_{\mathrm{P}} & n(\mathrm{~A})^{-1} \\
& \times \int_{\Pi(\mathrm{M})} \int_{\mathrm{G}(\mathbb{Q}) \backslash \mathrm{G}(\mathbb{A})}\left(\sum_{\phi \in \mathcal{B}_{\mathrm{P}}(\pi)_{\chi}} E(x, \phi) \overline{E(y, \phi)}\right) F(y) d y d \pi \\
= & \sum_{\chi \in \mathfrak{X}} \sum_{\mathrm{P}} n(\mathrm{~A})^{-1} \int_{\Pi(\mathrm{M})} \int_{\mathbf{G}(\mathbb{Q}) \backslash \mathrm{G}(\mathbb{A})}\left(\sum_{\phi \in \mathcal{B}_{\mathrm{P}}(\pi)_{\chi}} E(x, \phi) \overline{E(y, \phi)}\right) \\
\times\left(R\left(\eta_{1}\right) \Delta^{n} F(y)+R\left(\eta_{2}\right) F(y)\right) d y d \pi .
\end{array}
$$

Consequently, $S^{\prime}(F, x)$ will be the sum of the following two series:

$$
\begin{array}{rl}
S_{1}(F, x)=\sum_{\chi \in \mathcal{X}} \sum_{\mathrm{P}} & n(\mathrm{~A})^{-1} \int_{\Pi(\mathrm{M})} \int_{\mathrm{G}(\mathbb{Q}) \backslash \mathrm{G}(\mathbb{A})}\left(\sum_{\phi \in \mathcal{B}_{\mathrm{P}}(\pi)_{\chi}} E(x, \phi) \overline{E\left(y, I_{\mathrm{P}}\left(\pi, \tilde{\eta}_{1}\right) \phi\right)}\right) \\
\times \Delta^{n} F(y) d y d \pi
\end{array}
$$

and

$$
\begin{array}{rl}
S_{2}(F, x)=\sum_{\chi \in \mathcal{X}} \sum_{\mathrm{P}} & n(\mathrm{~A})^{-1} \int_{\Pi(\mathrm{M})} \int_{\mathrm{G}(\mathbb{Q}) \backslash \mathrm{G}(\mathbb{A})}\left(\sum_{\phi \in \mathcal{B}_{\mathrm{P}}(\pi)_{\chi}} E(x, \phi) \overline{E\left(y, I_{\mathrm{P}}\left(\pi, \tilde{\eta}_{2}\right) \phi\right)}\right) \\
\times F(y) d y d \pi &
\end{array}
$$

for an appropriately chosen compactly supported function $\tilde{\eta}_{1}, \tilde{\eta}_{2}$. By Lemma 4.4 of [1, there are $N, r_{0}$ and a continuous seminorm $\|\cdot\|_{r_{0}}$ on $C_{c}^{r_{0}}(\mathrm{G}(\mathbb{A}))$ such that if 
$r \geq r_{0}$ and $\eta$ is K-finite in $C_{c}^{r}(\mathrm{G}(\mathbb{A}))$,

$$
\sum_{\chi \in \mathfrak{X}} \sum_{\mathrm{P}} n(\mathrm{~A})^{-1} \int_{\Pi(\mathrm{M})}\left|\sum_{\phi \in \mathcal{B}_{\mathrm{P}}(\pi)_{\chi}} E(x, \phi) \overline{E\left(y, I_{\mathrm{P}}(\pi, \eta) \phi\right)}\right| d \pi
$$

is bounded by $\|\eta\|_{r_{0}} \cdot\|x\|^{N} \cdot\|y\|^{N}$. Here we have defined the height $\|\cdot\|$ on $G(\mathbb{A})$ as in [1, page 918. Since $m$ can be taken to be arbitrarily large, all we need to verify in order to get the uniform convergence is the convergence of the following integrals:

$$
\int_{G(\mathbb{A})}|f(y)| \cdot\|y\|^{N} d y
$$

and

$$
\int_{G(\mathbb{A})}\left|\Delta^{n} f(y)\right| \cdot\|y\|^{N} d y
$$

We summarize this discussion:

Lemma 3.1. Let $n, N$ be as above. Assuming convergence of the integrals (3.27) and (3.28) we have

$$
F(x)=S_{1}(F, x)+S_{2}(F, x) .
$$

3.3. Truncations. Let $T \in \mathfrak{a}_{\mathrm{B}}^{+}$. Let $\Delta_{0}(\mathrm{~B}, \mathrm{~A})$ be a set of simple roots of $(\mathrm{B}, \mathrm{A})$, and $\mathrm{d}_{\mathrm{B}}(T):=\min _{\alpha \in \Delta_{0}(\mathrm{~B}, \mathrm{~A})}\{\alpha(T)\}$. We will need those $T$ which are sufficiently regular; this means that $\mathrm{d}_{\mathrm{B}}(T)$ is large. Recall that for sufficiently regular $T$, the Arthur truncation $\wedge^{T}$ acts on functions on $G(\mathbb{Q}) \backslash G(\mathbb{A})[2$.

Lemma 3.2. (1) If $\phi$ is locally bounded, then $\wedge^{T} \phi$ is defined everywhere.

(2) If $\phi: G(\mathbb{Q}) \backslash \mathrm{G}(\mathbb{A}) \rightarrow \mathbb{C}$ is locally $\mathrm{L}^{1}$, then we have

$$
\wedge^{T} \wedge^{T} \phi(g)=\wedge^{T} \phi(g)
$$

for almost all $g$. If $\phi$ is locally bounded, then the identity holds for all $g$.

(3) Suppose $\phi_{1}, \phi_{2}: \mathrm{G}(\mathbb{Q}) \backslash \mathrm{G}(\mathbb{A}) \rightarrow \mathbb{C}$ are locally $\mathrm{L}^{1}$. If $\phi_{1}$ is of moderate growth and $\phi_{2}$ is rapidly decreasing, then

$$
\int_{\mathrm{G}(\mathbb{Q}) \backslash \mathrm{G}(\mathbb{A})} \overline{\wedge^{T} \phi_{1}(g)} \phi_{2}(g) d g=\int_{\mathrm{G}(\mathbb{Q}) \backslash \mathrm{G}(\mathbb{A})} \overline{\phi_{1}(g)} \wedge^{T} \phi_{2}(g) d g .
$$

Observe that the lemma implies that if $\phi_{1}, \phi_{2}$ are as above, then

$$
\int_{\mathrm{G}(\mathbb{Q}) \backslash \mathrm{G}(\mathbb{A})} \overline{\wedge^{T} \phi_{1}(g)} \phi_{2}(g) d g=\int_{\mathrm{G}(\mathbb{Q}) \backslash \mathrm{G}(\mathbb{A})} \overline{\wedge^{T} \phi_{1}(g)} \wedge^{T} \phi_{2}(g) d g .
$$

Given $\mathrm{P}$ and $\pi \in \Pi\left(\mathrm{M}_{\mathrm{P}}(\mathrm{A})\right)$ and $\lambda \in i \mathfrak{a}_{\mathrm{P}}^{*}$, we define an operator

$$
\Omega_{\chi, \pi}^{T}(\mathrm{P}, \lambda): \mathcal{H}_{\mathrm{P}}^{0}(\pi)_{\chi} \rightarrow \mathcal{H}_{\mathrm{P}}^{0}(\pi)_{\chi}
$$

by setting

$$
\left(\Omega_{\chi, \pi}^{T}(\mathrm{P}, \lambda) \pi, \pi^{\prime}\right)=\int_{\mathrm{G}(\mathbb{Q}) \backslash \mathrm{G}(\mathbb{A})^{1}} \wedge^{T} E(x, \phi, \lambda) \overline{\wedge^{T} E\left(x, \phi^{\prime}, \lambda\right)} d x,
$$

for each pair of vectors $\phi, \phi^{\prime} \in \mathcal{H}_{\pi}^{0}(\pi)_{\chi}$.

Next let $\mathrm{K}_{0}$ be a subgroup of finite index in $\mathrm{K}_{f}$. Suppose that $W$ is a finite dimensional representation of $\mathrm{K}_{\infty}$. Given $\mathrm{P} \supset \mathrm{B}, \pi \in \mathrm{P}\left(\mathrm{M}_{\mathrm{P}}(\mathbb{A})^{1}\right)$, let $\mathcal{H}_{\mathrm{P}}^{0}(\pi)_{\chi}^{\mathrm{K}_{0}}$ be the space of $\mathrm{K}_{0}$-invariant functions in $\mathcal{H}_{\mathrm{P}}^{0}(\pi)_{\chi}$, and let $\mathcal{H}_{\mathrm{P}}^{0}(\pi)_{\chi}^{\mathrm{K}_{0}, W}$ be the subspace 
of functions in $\mathcal{H}_{\mathrm{P}}^{0}(\pi)_{\chi}^{\mathrm{K}_{0}}$ which transform under $\mathrm{K}_{\infty}$ according to $W$. If a linear operator $A$ on $\mathcal{H}_{\mathrm{P}}^{0}(\pi)_{\chi}$ leaves any of these subspaces $\mathcal{H}_{\mathrm{P}}^{0}(\pi)_{\chi}^{\square}$, with $\square=\mathrm{K}_{0}$ or $\square=\mathrm{K}_{0}, W$, invariant, we denote by $A_{*}$ the restriction of $A$ to the appropriate subspace. Choose the differential operator $\Delta$ as in the proof of Lemma 4.1 of [1]. Then $\Delta$ acts on $\mathcal{H}_{\mathrm{P}}^{0}(\pi)_{\chi}$ through each of the representations $I_{\mathrm{P}}\left(\pi_{\zeta}\right)$, and we denote the action by $I_{\mathrm{P}}\left(\pi_{\zeta}, \Delta\right)$. This action leaves the two subspaces mentioned above invariant. For $\zeta \in i \mathfrak{a}_{\mathrm{P}}^{*}$, the action of $I_{\mathrm{P}}\left(\pi_{\zeta}, \Delta\right)$ is via a scalar greater than 1 .

Lemma 3.3 (Arthur [3]). There exist integers $C_{0}, d_{0}$, and $m$ such that for any subgroup $\mathrm{K}_{0} \subset \mathrm{K}_{f}$ of finite index, and any $T \in i \mathfrak{a}_{\mathrm{B}}^{+}$with $\mathrm{d}_{\mathrm{B}}(T)>C_{0}$, the expression

$$
\sum_{\chi \in \mathfrak{X}} \sum_{\mathrm{P}} \sum_{\pi \in \Pi\left(\mathrm{M}_{\mathrm{P}}(\mathbb{A})^{1}\right)}|n(\mathrm{~A})|^{-1} \int_{i \mathfrak{a}_{\mathrm{P}}^{*} / i \mathfrak{a}_{\mathrm{G}}^{*}}\left\|\Omega_{\chi, \pi}^{T}(\mathrm{P}, \zeta)_{\mathrm{K}_{0}} \cdot I_{\mathrm{P}}\left(\pi_{\zeta}, \Delta^{m}\right)^{-1}\right\|_{1} d \zeta
$$

is bounded by $C_{\mathrm{K}_{0}}(1+\|T\|)^{d_{0}}$. Here $C_{\mathrm{K}_{0}}$ is a constant which depends only on $\mathrm{K}_{0}$, and $\|\cdot\|_{1}$ is the trace class norm.

3.4. A bound for Eisenstein series. We recall a bound for Eisenstein series embedded in the proof of Proposition 2 of [26]. Fix a large compact set $C$ of $\mathrm{G}(\mathbb{A})$ and a small compact open subgroup $\mathrm{K}$ of $\mathrm{G}\left(\mathbb{A}_{f}\right)$, and let $f$ be a sufficiently differentiable function with support in $C$.

Lemma 3.4. Fix a compact set $C^{\prime}$ in $\mathrm{G}(\mathbb{A})$. There are constants $c, N>0$ depending only on the support of $f$ such that for sufficiently regular $T$ we have

$$
\left|E\left(g, I\left(f, \pi_{\lambda}\right) \varphi, \lambda\right)\right| \leq c\|g\|^{N}\|f\|_{\infty} \cdot\left\|\wedge^{T} E(\cdot, \varphi, \lambda)\right\|_{L^{2}(\mathrm{G}(\mathbb{Q}) \backslash \mathrm{G}(\mathbb{A}))} .
$$

This lemma combined with Lemma 3.3 together with Lemma 4.1 of [1] implies the following result.

Proposition 3.5. For $\phi \in \mathcal{B}_{\mathrm{P}}(\pi)_{\chi}$ let $\Lambda(\phi)$ be defined by $\Delta \cdot \phi=\Lambda(\phi) \cdot \phi$. Then there is an $m>0$ such that

$$
\sum_{\chi \in \mathcal{X}} \sum_{\mathrm{P}} n(\mathrm{~A})^{-1} \int_{\Pi(\mathrm{M})}\left(\sum_{\phi \in \mathcal{B}_{\mathrm{P}}(\pi)_{\chi}} \Lambda(\phi)^{-m}|E(e, \phi)|^{2}\right) d \pi
$$

is convergent. The outermost summation is only over those classes for which $\Lambda(\phi) \neq 0$ are fixed by $\mathrm{K}_{0}$.

\section{Spherical functions and bounds for matrix COEFFicients}

4.1. Spherical functions. Let $\pi$ be an infinite-dimensional automorphic representation of $\mathrm{G}$, and suppose $\phi$ is a right K-finite automorphic form in the space of $\pi$. Here $\mathrm{K}=\prod_{v} \mathrm{~K}_{v}$. Define a function $M(\phi, g)$ on $\mathrm{G}(\mathbb{A})$ by

$$
M(\phi, g)=\frac{1}{\operatorname{vol}(\mathrm{K})^{2}} \int_{\mathrm{K}} \int_{\mathrm{K}} \phi\left(\kappa g \kappa^{\prime}\right) d \kappa d \kappa^{\prime} .
$$

If $\pi$ has no $\mathrm{K}$-invariant vectors, then the above integral is zero, and we may assume that $\phi$ is right $K$ invariant. Let $V_{\pi}$ be a vector space on which $G(\mathbb{A})$ acts via a representation which is isomorphic to the space of K-finite vectors. Let $j: \mathrm{V}_{\pi} \rightarrow \mathrm{H}_{\pi}$ be the intertwining map. We have used the same notation for a representation and its underlying representations space. By a standard result of Jacquet, Langlands, and Flath, we have

$$
\mathrm{V}_{\pi}=\bigotimes_{v} \mathrm{~V}_{\pi, v}
$$


Since $\phi$ is right K-finite, it is in the image of $j$. Let $w=j^{-1}(\phi)$. Then

$$
w \in \mathrm{V}_{\pi}^{\mathrm{K}}=\left(\bigotimes_{v \notin S_{\infty}} \mathrm{V}_{\pi, v}^{\mathrm{K}_{v}}\right) \otimes\left(\bigotimes_{v \in S_{\infty}} \mathrm{V}_{\pi, v}\right) .
$$

Set $S=S_{F}$. We know that for $v \notin S$ we have $\operatorname{dim}\left(\mathrm{V}_{\pi, v}^{\mathrm{K}_{v}}\right)=1$. Fix a non-zero element $e_{v}$ in each of these spaces. This means then

$$
w=\left(\bigotimes_{v \notin S} e_{v}\right) \otimes w_{S}
$$

where

$$
w_{S} \in\left(\bigotimes_{v \in S \backslash S_{\infty}} \mathrm{V}_{\pi, v}^{\mathrm{K}_{v}}\right) \otimes\left(\bigotimes_{v \in S_{\infty}} \mathrm{V}_{\pi, v}\right) .
$$

Next we examine $M(\phi, g)$. Define a functional $\lambda$ on $\mathrm{V}_{\pi}$ by

$$
\lambda(\nu)=\int_{\mathbf{K}} j(\nu)(\kappa) d \kappa
$$

(for $\nu \in \mathrm{V}_{\pi}$ ). Then it is easily seen that

$$
\lambda \in \bigotimes_{v \notin S} \widetilde{\mathrm{V}}_{\pi, v}^{\mathrm{K}_{v}} \otimes \mathrm{V}_{S}^{*}
$$

Here $\mathrm{V}_{S}=\bigotimes_{v \in S} \mathrm{~V}_{\pi, v}, \mathrm{~V}_{S}^{*}$ is the dual space and $\widetilde{\mathrm{V}}_{\pi}$ is the smooth dual of the local representation $\mathrm{V}_{\pi}$. Since the smooth dual of an admissible representation is admissible, it follows that $\operatorname{dim}\left(\widetilde{\mathrm{V}}_{\pi, v}^{\mathrm{K}_{v}}\right)=1$. For each $v \notin S$ choose an element $\xi_{v}$ in this space in such a way that $\xi_{v}\left(e_{v}\right)=1$. This then means that, as in the case of $w=j^{-1}(\phi)$, we have

$$
\lambda=\left(\bigotimes_{v \notin S} \xi_{v}\right) \otimes \lambda_{S}
$$

with $\lambda_{S}$ in the obvious space. Combining this identity with the similar identity for $w$ we obtain

$$
\begin{aligned}
\lambda(v) & =\left(\prod_{v \notin S} \xi_{v}\left(e_{v}\right)\right) \cdot \lambda_{S}\left(w_{S}\right) \\
& =\lambda_{S}\left(w_{S}\right) .
\end{aligned}
$$

This implies that

$$
\begin{aligned}
\lambda_{S}\left(w_{S}\right) & =\int_{\mathbf{K}} j(w)(\kappa) d \kappa \\
& =\int_{K^{S}} j(w)(\kappa) d \kappa .
\end{aligned}
$$

Here $\mathrm{K}^{S}=\prod_{v \in S} \mathrm{~K}_{v}$. Consider an embedding $\eta: \mathrm{G}\left(\mathbb{A}_{S}\right) \longrightarrow \mathrm{G}(\mathbb{A})$ given by

$$
g \mapsto(1,1, \ldots, 1, g) .
$$

Obviously,

$$
\begin{aligned}
\operatorname{vol}\left(\mathrm{K}^{S}\right) M(\phi, g) & =\lambda(\pi(g) w) \\
& =\prod_{v \notin S} \varphi_{v, \pi}\left(g_{v}\right) \cdot \int_{\mathrm{K}^{S}} \phi\left(\kappa \eta\left(g_{S}\right)\right) d \kappa
\end{aligned}
$$


where $\varphi_{v, \pi}\left(g_{v}\right)=\xi_{v}\left(\pi_{v}\left(g_{v}\right) e_{v}\right)$ is the normalized local spherical function.

Corollary 4.1. If $\mathrm{K}=\prod_{v} \mathrm{~K}_{v}$ is such that for each local place $v$, including the archimedean places, the local compact subgroups $\mathrm{K}_{v}$ satisfy hypotheses I and II of [36], then

$$
M(\phi, g)=\phi(e) \prod_{v} \varphi_{v, \pi}\left(g_{v}\right)
$$

if each $\pi$ has a $\mathrm{K}_{v}$-fixed vector; otherwise it is zero.

We note that local $\mathrm{K}_{v}$ satisfying I and II of [36] exist by [20, for archimedean places, and [10, 39, 41], for non-archimedean places.

4.2. Bounds on matrix coefficients. In this section we recall an important result of $\mathrm{H}$. Oh that is used in estimates leading to the proof of the spectral expansion (3.22).

Let $k$ be a non-archimedean local field of $\operatorname{char}(k) \neq 2$, and residual degree $q$. Let $\mathrm{H}$ be the group of $k$-rational points of a connected reductive split or quasi-split group with $\mathrm{H} / \mathrm{Z}(\mathrm{H})$ almost $k$-simple. Let $\mathrm{S}$ be a maximal $k$-split torus, $\mathrm{B}$ a minimal parabolic subgroup of $\mathrm{H}$ containing $\mathrm{S}$ and $\mathrm{K}$ a good maximal compact subgroup of $\mathrm{H}$ with Cartan decomposition $\mathrm{G}=\mathrm{KS}(k)^{+} \mathrm{K}$. Let $\Phi$ be the set of non-multipliable roots of the relative root system $\Phi(\mathrm{H}, \mathrm{S})$, and $\Phi^{+}$the set of positive roots in $\Phi$. A subset $\mathcal{S}$ of $\Phi^{+}$is called a strongly orthogonal system of $\Phi$ if any two distinct elements $\alpha$ and $\alpha^{\prime}$ of $\mathcal{S}$ are strongly orthogonal, that is, neither of $\alpha \pm \alpha^{\prime}$ belongs to $\Phi$. Define a bi-K-invariant function $\xi_{\mathcal{S}}$ on $\mathrm{H}$ as follows: first set

then

$$
n_{\mathcal{S}}(g)=\frac{1}{2} \sum_{\alpha \in \mathcal{S}} \log _{q}|\alpha(g)|
$$

$$
\xi_{\mathcal{S}}(g)=q^{-n_{\mathcal{S}}(g)} \prod_{\alpha \in \mathcal{S}}\left(\frac{\left(\log _{q}|\alpha(g)|\right)(q-1)+(q+1)}{q+1}\right) .
$$

Theorem 4.2 (29, Theorem 1.1). Assume that the semi-simple $k$-rank of $\mathrm{H}$ is at least 2 and let $\mathcal{S}$ be a strongly orthogonal system of $\Phi$. Then for any unitary representation @ of $\mathrm{H}$ without an invariant vector and with $\mathrm{K}$-finite unit vectors $\nu$ and $\nu^{\prime}$, one has

$$
\left|\left(\varrho(g) \nu, \nu^{\prime}\right)\right| \leq\left(\operatorname{dim}(\mathrm{K} \nu) \operatorname{dim}\left(\mathrm{K} \nu^{\prime}\right)\right)^{\frac{1}{2}} \cdot \xi_{\mathcal{S}}(g),
$$

for any $g \in \mathrm{H}$.

Corollary 4.3. Let $v \notin S_{F}$. Let $\varphi_{v}$ be the normalized spherical function associated with an infinite dimensional unramified principal series representation of $\mathrm{G}\left(F_{v}\right)$. Suppose that the semi-simple rank of $\mathrm{G}\left(F_{v}\right)$ is at least 2. Then for each $\vartheta \in \Delta\left(\mathrm{G}\left(F_{v}\right), \mathrm{S}_{v}\left(F_{v}\right)\right)$ we have

$$
\left|\varphi_{v}\left(\check{\vartheta}\left(\varpi_{v}\right)\right)\right|<2 q_{v}^{-\frac{\ell_{v}(\vartheta)}{2}} .
$$

Proof. Fix $\vartheta$. By definition, a singleton is a strongly orthogonal set. Set

$$
\mathcal{S}_{v}=\{\vartheta\}
$$

Then

$$
n_{\mathcal{S}_{v}}\left(\check{\vartheta}\left(\varpi_{v}\right)\right)=\frac{\ell_{v}(\vartheta)}{2}
$$


and

$$
\xi_{\mathcal{S}_{p}}\left(\check{\vartheta}\left(\varpi_{v}\right)\right)=q_{v}^{-\frac{\ell_{v}(\vartheta)}{2}} \frac{2 q_{v}}{q_{v}+1} .
$$

Since $\nu$ and $\nu^{\prime}$ that define the spherical function are $\mathrm{K}_{v}$-invariant, this equation combined with Theorem 4.2 implies

$$
\begin{aligned}
\mid \varphi_{v}\left(\check{\vartheta}\left(\varpi_{v}\right)\right) & \leq \xi_{\mathcal{S}_{v}}\left(\check{\vartheta}\left(\varpi_{v}\right)\right) \\
& \leq q^{-\frac{\ell_{v}(\vartheta)}{2}} \frac{2 q_{v}}{q_{v}+1} \\
& <2 q_{v}^{-\frac{\ell_{v}(\vartheta)}{2}}
\end{aligned}
$$

which gives the claim.

We also need a similar bound on spherical functions when the semi-simple rank is equal to one. In this case, local considerations do not suffice, as the trivial representation may not be isolated in the unitary dual of the local group. However, for our purposes it will suffice to obtain a bound for a restricted class of representations. Let $\pi$ be an infinite-dimensional unitary irreducible automorphic representation of G. Suppose $\pi=\bigotimes_{v} \pi_{v}$. Extending $S_{F}$ if necessary, we may assume that for $v \notin S_{F}$, $\pi_{v}$ is an unramified representation.

Proposition 4.4. For all $v \notin S_{F}$, the representation $\pi_{v}$ is infinite-dimensional.

Proof. Realize $\pi$ on a Hilbert subspace $\mathrm{V}$ of $\mathrm{L}^{2}(\mathrm{G}(F) \backslash \mathrm{G}(\mathbb{A}))$. Denote by $\mathrm{V}^{\infty}$ the subspace of $\mathrm{V}$ consisting of all vectors $\nu$ such that:

- $\nu$ is $\mathrm{K}_{f}$-finite, and

- for all archimedean places $v$, the map $\mathrm{G}\left(F_{v}\right) \rightarrow \mathrm{V}$ given by $g \mapsto \pi(g) \nu$ is $C^{\infty}$.

It is standard that $V^{\infty}$ is $G(\mathbb{A})$-stable, and dense in $V$ (with respect to the $L^{2}$ topology). The image of $V^{\infty}$ in $L^{2}$ consists of smooth functions. Suppose that for some $v \notin S_{F}$ the representation $\pi_{v}$ is not infinite-dimensional. Since this representation is admissible, it must be one-dimensional. Concretely, if we pick an element $\varphi \in \mathrm{V}^{\infty}$, we have

$$
\varphi\left(\gamma x_{v}\right)=\chi_{v}\left(x_{v}\right) \varphi(e), \quad \gamma \in \mathrm{G}(F), x_{v} \in \mathrm{G}\left(F_{v}\right) .
$$

Here $\chi_{v}: \mathrm{G}\left(F_{v}\right) \rightarrow \mathbb{C}^{\times}$is a one-dimensional representation of $\mathrm{G}\left(F_{v}\right)$. Next, by Satz 6.1. of 25] we know that the commutator subgroup $\mathrm{G}^{\prime}(\mathbb{A})$ of $\mathrm{G}(\mathbb{A})$ is contained in the closure of $\mathrm{G}(F) \mathrm{G}\left(F_{v}\right)$. As $\mathrm{G}^{\prime}(\mathbb{A})$ has no non-trivial one-dimensional representations, we conclude that for all $\varphi \in \mathrm{V}^{\infty}$ we have

$$
\varphi\left(g^{\prime}\right)=\varphi(e)
$$

for all $g^{\prime} \in \mathrm{G}^{\prime}(\mathbb{A})$. Since the subspace $\mathrm{V}^{\infty}$ is $\mathrm{G}(\mathbb{A})$-invariant, (4.2) must hold for all right translates of $\varphi$ by elements of $G(\mathbb{A})$. Hence

$$
\varphi(x g)=\varphi(g), \quad x \in \mathrm{G}^{\prime}(\mathbb{A}), g \in \mathrm{G}(\mathbb{A}) .
$$

Since $G^{\prime}(\mathbb{A})$ is a normal subgroup of $G(\mathbb{A})$, it follows that we must also have

$$
\varphi(g x)=\varphi(g), \quad x \in \mathrm{G}^{\prime}(\mathbb{A}), g \in \mathrm{G}(\mathbb{A}),
$$

i.e., every element of $\mathrm{V}^{\infty}$ is invariant under the restriction of the representation $\pi$ to the subgroup $\mathrm{G}^{\prime}(\mathbb{A})$. This implies that the representation $\pi$ on $\mathrm{V}^{\infty}$ factors 
through $G(\mathbb{A}) / G^{\prime}(\mathbb{A})$, which is an abelian group. Since irreducible admissible representations of abelian groups are all one-dimensional, the space $\mathrm{V}^{\infty}$ must be onedimensional. Finally, use the fact that $\mathrm{V}^{\infty}$ is dense in $\mathrm{V}$ to conclude that $\mathrm{V}$ is also one-dimensional.

Theorem 4.5. There is an absolute constant $c>0$ with the following property. Let $\pi=\bigotimes_{v} \pi_{v}$ be an infinite-dimensional irreducible automorphic representation of $\mathrm{G}$; for $\mathrm{G}=\mathrm{PGL}_{2}$, assume that $\pi$ is not the automorphic representation associated with an Eisenstein series induced from a Borel subgroup. Let $v$ be a place with $v \notin S_{F}$, and $\varphi_{v}$ the normalized spherical function of $\pi_{v}$. Then for all $\alpha \in \Delta\left(\mathrm{G}\left(F_{v}\right), \mathrm{S}_{v}\left(F_{v}\right)\right)$ we have

$$
\left|\varphi_{v}\left(\check{\vartheta}\left(\varpi_{v}\right)\right)\right|<q^{-c \ell_{v}(\vartheta)} .
$$

Proof. According to the result of Corollary 4.3, we recognize two cases:

The case where s.s. rank is equal to one. In this case, $\mathrm{G}$ is a form of $\mathrm{PGL}_{2}$ or $\mathrm{PGU}_{3}$. For the case of $\mathrm{PGL}_{2}$, if $\mathrm{G}$ is not split, then it has to be a quaternion algebra, and in this case by the Jacquet-Langlands correspondence, there is an irreducible cuspidal automorphic representation $\pi^{\prime}$ of $\mathrm{GL}_{2}$, such that for all $v \notin S$, we have $\pi_{v}=\pi_{v}^{\prime}$ as representations of $\mathrm{GL}_{2}\left(F_{v}\right)$. Since the local representation obtained this way is unramified, there must exist a pair of unramified quasi-characters $\chi$ and $\chi^{\prime}$ of $F_{v}^{\times}$such that $\pi_{v}^{\prime}=\pi\left(\chi, \chi^{\prime}\right)$. Since this representation has trivial central character, we must have $\chi^{\prime}=\chi^{-1}$. In this case, if $\alpha$ is the unique positive root, we have

$$
t_{v}(\alpha)=\left(\begin{array}{cc}
1 & \\
& \varpi_{v}
\end{array}\right)
$$

Next if $\chi \neq 1$, by the formula of Casselman ([13), we have

$$
\begin{aligned}
\varphi_{v}\left(\begin{array}{cc}
1 & \\
& \varpi_{v}
\end{array}\right) & =\frac{q^{-\frac{1}{2}}}{1+q^{-1}}\left\{\frac{1-q^{-1} \chi\left(\varpi_{v}\right)^{-2}}{1-\chi\left(\varpi_{v}\right)^{-2}} \chi\left(\varpi_{v}\right)+\frac{1-q^{-1} \chi\left(\varpi_{v}\right)^{2}}{1-\chi\left(\varpi_{v}\right)^{2}} \chi\left(\varpi_{v}\right)^{-1}\right\} \\
& =\frac{q^{-\frac{1}{2}}}{1+q^{-1}}\left(\chi\left(\varpi_{v}\right)+\chi\left(\varpi_{v}\right)^{-1}\right) .
\end{aligned}
$$

The same formula holds for $\chi=1$ by analytic continuation. Since $\chi$ is an unramified character, we have $\chi=|\cdot|^{s}$, for some complex number $s$. We now need some nontrivial estimate towards the Ramanujan conjecture. For example, by a recent result of Kim and Shahidi (24]), we know that

$$
-\frac{1}{6} \leq \Re(s) \leq \frac{1}{6} .
$$

This implies that

$$
\left|\varphi_{v}\left(\begin{array}{cc}
1 & \\
& \varpi_{v}
\end{array}\right)\right| \leq \frac{2 q^{-\frac{1}{2}+\frac{1}{6}}}{1+q^{-1}}<2 q^{-\frac{1}{3}} .
$$

When $\mathrm{G}=\mathrm{PGL}_{2}$ and $\pi$ is cuspidal, the result follows from the same result of Kim and Shahidi. Next, let $G$ be an inner form of the quasi-split group $G^{\prime}=\operatorname{PGU}(2,1)$. Suppose that $\mathrm{G}^{\prime}$ splits over a quadratic extension $E / F$. By Rogawski's theorem [34, there is an automorphic cuspidal representation $\pi^{\prime}=\bigotimes_{v} \pi_{v}^{\prime}$ of $\operatorname{PGU}(2,1)$ such that for all $v \notin S, \pi_{v}=\pi_{v}^{\prime}$ as representations of $\mathrm{G}^{\prime}\left(F_{v}\right)$. Consider the base change of $\pi^{\prime}$ from $\mathrm{PGU}(2,1) / F$ to $\mathrm{PGL}_{3} / E$, again established by Rogawski. At this point, use the result of $\mathrm{Oh}$ on $\mathrm{PGL}_{3}$. 
The case where the s.s. rank is larger than one. By Proposition 4.4 $\pi_{v}$ is not one-dimensional (for $v \notin S_{F}$ ), and the assertion follows from Corollary 4.3.

\section{Part 2. Geometry and height functions}

\section{Geometry}

In this section we recall the constructions and basic geometric properties of wonderful compactifications.

5.1. Flag varieties. An important class of varieties, homogeneous for the action of $G$, is the class of generalized flag varieties

$$
Y_{I}:=\mathrm{P}_{I} \backslash \mathrm{G} \text {. }
$$

The geometry of these, and their subvarieties (for example, Schubert varieties), plays an important role in different branches of algebra, e.g., representation theory and enumerative geometry.

We now recall some basic facts about these varieties. For $\lambda \in \mathfrak{X}^{*}\left(\mathbf{T}^{s c}\right)$ we can define a line bundle $L_{\lambda}$ on $\mathrm{B}^{s c} \backslash \mathrm{G}^{s c}=\mathrm{B} \backslash \mathrm{G}$ by

$$
\mathbf{G}^{s c} \times \mathbf{G}_{a} / \sim, \quad \text { with }(g, a) \sim\left(g b, \lambda^{-1}(b) a\right),
$$

$g \in \mathrm{G}^{s c}, b \in \mathrm{B}^{s c}$ and $a \in \mathbf{G}_{a}$. The canonical projection

$$
\pi: L_{\lambda} \rightarrow \mathrm{B} \backslash \mathrm{G}
$$

is given by

$$
\pi(g, a)=\mathrm{B} g
$$

This gives an identification of

$$
\operatorname{Pic}(Y)=\mathfrak{X}^{*}\left(\mathrm{~T}^{s c}\right) .
$$

Under this identification, the (closures of the) ample and the effective cones of $Y$ correspond to the positive Weyl chamber, that is, the set of non-negative linear combinations of $\omega_{i}$. The anticanonical class is given by

$$
-K_{Y}=2 \rho .
$$

5.2. Wonderful compactifications. First we work over an algebraically closed field of characteristic zero.

Proposition 5.1 (16, 12]). There exists a canonical compactification of a connected adjoint group $\mathrm{G}$ : a smooth projective variety $X$ such that

- $\mathrm{G} \subset X$ is a Zariski open subvariety, and the action of $\mathrm{G} \times \mathrm{G}$ on $\mathrm{G}$, by

$$
\left(g_{1}, g_{2}\right)(g)=g_{1} g g_{2}^{-1},
$$

extends to an action of $\mathrm{G} \times \mathrm{G}$ on $X$.

- The boundary $X \backslash \mathrm{G}$ is a union of strict normal crossings divisors $D_{i}$ (for $i=1, \ldots, r)$. For every $I \subset[1, \ldots, r]$ the subvariety $D_{I}=\bigcap_{i \in I} D_{i}$ is a $\mathrm{G} \times \mathrm{G}$-orbit closure. All $\mathrm{G} \times \mathrm{G}$-orbit closures are obtained this way.

- $X$ contains a unique closed $\mathrm{G} \times \mathrm{G}$-orbit $Y=\mathrm{G} / \mathrm{B} \times \mathrm{G} / \mathrm{B}$.

- The components $D_{I}$ are isomorphic to fibrations over $\mathrm{G} / \mathrm{P}_{I} \times \mathrm{G} / \mathrm{P}_{I}$ with fibers canonical compactifications of the adjoint form of the associated Levi groups.

We review several constructions of wonderful compactifications over algebraically closed fields (of characteristic 0); throughout $\mathrm{G}$ is semi-simple adjoint: 
Via Hilbert schemes. Let $\mathrm{P} \subset \mathrm{G}$ be a parabolic subgroup and $Y=\mathrm{G} / \mathrm{P}$ the associated flag variety. Then $X$ is the $\mathrm{G} \times \mathrm{G}$-orbit closure of the diagonal of the Hilbert scheme of $Y \times Y$ (see [1]).

Via representations. Let $\lambda$ be a regular dominant weight of $\mathrm{G}$ and $V_{\lambda}$ the irreducible representation of $\mathrm{G}$ with highest weight $\lambda$. We have an action of $\mathrm{G} \times \mathrm{G}$ on

$$
\operatorname{End}\left(V_{\lambda}\right)=V_{\lambda} \otimes V_{\lambda}^{*}
$$

Taking the closure of the orbit through (the image of) the identity in $\mathbb{P}\left(\operatorname{End}\left(V_{\lambda}\right)\right)$ we obtain the canonical compactification $X$.

Via Lie algebras. Let $\mathfrak{g}=\operatorname{Lie}(G)$ and $n=\operatorname{dim}(G)=\operatorname{dim}(\mathfrak{g})$. The variety $\mathbb{L}$ of Lie subalgebras of the Lie algebra $\mathfrak{g} \oplus \mathfrak{g}$ can be regarded as a subvariety of the Grassmannian $\operatorname{Gr}(n, 2 n)$. It contains $\mathfrak{g}$, embedded diagonally. Moreover, $\mathbb{L}$ is a projective $\mathrm{G} \times \mathrm{G}$-variety. Taking the closure of the $\mathrm{G} \times \mathrm{G}$-orbit $X^{\circ}$ through $\mathfrak{g}$ we obtain $X \subset \mathbb{L}$. Since $\mathrm{G}$ is an adjoint group, the adjoint representation of $\mathrm{G}$ on $\mathrm{G} \times \mathrm{G}$ is faithful and we may identify $X^{\circ}$ with the variety $\mathrm{G}$, or more precisely $\mathrm{G} \times \mathrm{G} / \operatorname{Diag}(\mathrm{G})$.

We proceed to describe the boundary $X \backslash G$ in the latter representation. Let $\mathrm{P}=\mathrm{P}_{I}$ (with $I \subset[1, \ldots, r]$ ) be a standard parabolic subgroup of $\mathrm{G}$. Choose a Levi decomposition $\mathrm{P}=\mathrm{M} \cdot \mathrm{U}$. Let $\mathfrak{L}_{P}$ be the set of pairs

$$
\left(m+u, m+u^{\prime}\right) \text {, with } m \in \operatorname{Lie}(\mathrm{M}), u, u^{\prime} \in \operatorname{Lie}(\mathrm{U}) .
$$

Then $\mathfrak{L}_{\mathrm{P}}$ is a subalgebra of $\mathfrak{g} \oplus \mathfrak{g}$. Next let $\mathcal{C}=\{\mathrm{P}\}$ denote the conjugacy class of parabolic subgroups of $G$ containing $P$. We note that the $G \times G$-orbit of $\mathfrak{L}_{P}$ in $\mathbb{L}$ does not depend on the particular choice of $\mathrm{M}$ in the Levi decomposition of $\mathrm{P}$; in fact, it depends only on the class $\{\mathrm{P}\}$. We denote this orbit by $D_{\mathcal{C}}$. When $\mathrm{P}$ is maximal, the orbit $D_{\mathcal{C}}$ is a smooth irreducible divisor in $X$. Moreover,

$$
X \backslash G=\bigcup_{\mathcal{C}} D_{\mathcal{C}},
$$

the union over classes of maximal parabolics. As the classes $\mathcal{C}$ of maximal parabolics in $\mathrm{G}$ are in bijection with the simple roots $\alpha$ of $\mathrm{T}$, we may then write

$$
D_{\mathcal{C}}=D_{i},
$$

if $\mathcal{C}$ is the maximal parabolic that corresponds to $\alpha_{i}$.

5.3. The Picard group of $X$. For $\mathrm{w} \in \mathrm{W}$ we denote by $X(\mathrm{w})$ the closure of $\mathrm{BwB} \subset \mathrm{G}$ in $X$. The $\mathrm{B} \times \mathrm{B}$-stable boundary components $D_{i}$ correspond to $X\left(s \mathrm{w}_{0} s_{i}\right)$. Every line bundle $L$ on $X$ restricts to the unique closed $\mathrm{G} \times \mathrm{G}$-orbit $Y=\mathrm{G} / \mathrm{B} \times \mathrm{G} / \mathrm{B}$; we get a restriction map $\operatorname{Pic}(X) \rightarrow \operatorname{Pic}(Y)$. Recall that in Section 5.1 we have identified the Picard group of $\mathrm{G} / \mathrm{B}$ with $\mathfrak{X}^{*}\left(\mathrm{~T}^{s c}\right)$.

Proposition 5.2. Let $X$ be the canonical compactification of $\mathrm{G}$ as above.

- The Picard group $\operatorname{Pic}(X)$ is freely generated by the classes $\left[D_{i}\right]$.

- The image of $\operatorname{Pic}(X) \hookrightarrow \operatorname{Pic}(Y)$ consists of classes

$$
L(\lambda)=\left(\lambda,-\mathrm{w}_{0} \lambda\right) \subset \operatorname{Pic}(Y)=\operatorname{Pic}(\mathrm{G} / \mathrm{B}) \times \operatorname{Pic}(\mathrm{G} / \mathrm{B}) .
$$


- The (closed) cone of effective divisors is given by

$$
\Lambda_{\mathrm{eff}}(X):=\bigoplus_{i=1}^{\mathrm{r}} \mathbb{R}_{\geq 0}\left[D_{i}\right]
$$

More precisely, if $\lambda \in \mathfrak{X}\left(\mathrm{T}^{s c}\right)$ is a dominant weight, then the line bundle $L(\lambda)$ on $X$ has a unique (up to scalars) global section $f_{\lambda}$ with divisor

$$
\operatorname{div}\left(f_{\lambda}\right)=\sum_{i=1}^{\mathrm{r}}\left\langle\lambda, \alpha_{i}^{\vee}\right\rangle D_{i}
$$

Moreover, $f_{\lambda}$ is an eigenvector of $\mathrm{B}^{s c} \times \mathrm{B}^{s c}$ with weight $\left(-\mathrm{w}_{0} \lambda, \lambda\right)$.

- The anticanonical class is given by

$$
-K_{X}=L\left(2 \rho+\sum_{i=1}^{r} \alpha_{i}\right) .
$$

Proof. See [16] and [12].

5.4. Line bundles. Let $L$ be a line bundle on $X$. Then $L$ admits a G-linearization there exists a $\mathrm{G}^{s c} \times \mathrm{G}^{s c}$-action on $\pi: L \rightarrow X$ such that for $\left(g_{1}, g_{2}\right) \in \mathrm{G}^{s c} \times \mathrm{G}^{s c}, l \in L$, one has

$$
\pi\left(\left(g_{1}, g_{2}\right) \circ l\right)=\left(\bar{g}_{1}, \bar{g}_{2}\right) \circ \pi(l),
$$

where $\bar{g}$ is the image of $g \in \mathrm{G}^{s c}$ in $\mathrm{G}$. We have a representation $\rho=\rho_{L}$ of $\mathrm{G}^{s c} \times \mathrm{G}^{s c}$ on the space of global sections $H^{0}(X, L)$.

Theorem 5.3. Let $\lambda \in \mathfrak{X}^{*}\left(\mathrm{~T}^{s c}\right)$ and $L(\lambda)$ be the associated line bundle on $X$. Let $\rho_{\lambda}$ be the representation of $\mathrm{G}^{s c} \times \mathrm{G}^{s c}$ on $H^{0}(X, L(\lambda))$. Then:

- The representation $\rho_{\lambda}$ decomposes with multiplicity one.

- Let $\gamma$ be the dominant weight on $\mathrm{T}^{s c}$ (relative to $\mathrm{B}^{s c}$ ). Let $\xi_{\gamma}$ be the associated irreducible representation of $\mathrm{G}^{s c}$ and $\eta_{\gamma}$ the irreducible representation of $\mathrm{G}^{s c} \times \mathrm{G}^{s c}$ defined by

$$
\eta_{\gamma}=\xi_{\gamma} \times \xi_{\gamma}^{*}
$$

Then each irreducible component of $\rho_{\lambda}$ is of the form $\eta_{\gamma}$ for some dominant weight $\gamma$ of $\mathrm{T}^{s c}$. Moreover, if $\eta_{\gamma}$ appears in $\rho_{\lambda}$, then $\gamma$ has the form

$$
\gamma=\lambda-\sum_{i=1}^{r} n_{i} \lambda_{i}
$$

with $n_{i} \in \mathbb{Z}_{\geq 0}$, for all $i=1, \ldots, r$.

- In particular, the restriction of $\rho_{\lambda}$ to $\mathrm{G}^{s c} \times\{1\}$ is a sum, with multiplicity, of $\xi_{\gamma}$ 's with $\gamma$ as above.

5.5. Non-split forms. We now return to the case of non-split $G$ and discuss the canonical $F$-structure on the wonderful compactification $X$ of $\mathrm{G}$ as well as the corresponding Galois action on the boundary divisors $D_{\alpha}$, for $\alpha \in \Delta$.

A summary can be given as follows: in the Lie algebra model, the Galois group $\Gamma$ operates on $\operatorname{Gr}(n, 2 n)$ in an obvious way and preserves $\mathbb{L}$ and $X^{\circ}$. Hence $\Gamma$ operates on $X$. Since $\Gamma$ acts on the parabolic subgroups of $\mathrm{G}$, it also permutes the boundary 
divisors

$$
\sigma\left(D_{\alpha}\right)=D_{\sigma(\alpha)}
$$

Here if $\mathcal{C}=\{\mathrm{P}\}$, then $\sigma(\mathcal{C})=\{\sigma(\mathrm{P})\}$.

The $F$-group $\mathrm{G}^{\prime}$ is defined by a homomorphism

$$
\theta: \Gamma \rightarrow \operatorname{Out}\left(\mathrm{G}^{s p}\right)
$$

to the group of outer automorphisms relative to the pair $\left(\mathrm{B}^{s p}, \mathrm{~T}^{s p}\right)$ and $\mathrm{B}^{s p}, \mathrm{~T}^{s p}$ chosen as above and both defined over $F$. We then have an action of $\Gamma$ on $\Delta$. Moreover,

$$
\sigma\left(D_{\alpha}\right)=D_{\sigma(\alpha)} \text { for } \alpha \in \Delta
$$

Finally, since $G$ is obtained $G^{\prime}$ by inner twisting, the two actions of $\Gamma$ on the classes of maximal parabolics in $\mathrm{G}(E)$ and $\mathrm{G}^{\prime}(E)$ coincide.

In detail, let $\lambda=\sum_{i=1}^{r} \omega_{i}$ and $V_{\lambda}$ be the corresponding representation with highest weight $\lambda$. We first regard $V_{\lambda}$ as a module for $\mathrm{G}^{\prime}$. As such, it is defined over $F$, since $\lambda \in \mathfrak{X}^{*}\left(\mathrm{~T}^{\prime}\right)_{F}$ (the action of $\Gamma$ on $\mathfrak{X}^{*}\left(\mathrm{~T}^{\prime}\right)=\mathfrak{X}^{*}(\mathrm{~T})$ via $\theta$ simply permutes the $\left.\omega_{i}\right)$. Let $L_{2 \lambda}$ be the corresponding line bundle on $\mathrm{B}^{\prime} \backslash \mathrm{G}^{\prime}$. Then $W:=H^{0}\left(\mathrm{~B}^{\prime} \backslash \mathrm{G}^{\prime}, L_{2 \lambda}\right)$ has an $F$-structure $W_{F}$. Denote by $\rho=\rho_{\lambda}$ the absolute representation of $\mathrm{G}$ on $W$. By the Borel-Weil theorem, we may identify $V_{2 \lambda}$ with $W_{F} \otimes_{F} \bar{F}$; this gives an action of $\Gamma$ on $V_{2 \lambda}$ :

$$
\sigma(w \otimes a)=w \otimes \sigma(a), \quad \text { for } w \in W_{F}, a \in \bar{F} .
$$

We have the twisted action $\tilde{\sigma}=c(\sigma) \cdot \sigma$ of $\Gamma$ on $\mathrm{G}(\bar{F})$; the corresponding twisted action on $W \otimes W^{*}$ is given by

$$
\tilde{\sigma} \cdot w=\rho \otimes \rho^{*}(c(\sigma), c(\sigma)) \cdot \sigma .
$$

If we identify $\operatorname{End}(W)=W \otimes W^{*}$ we see that this action is

$$
\tilde{\sigma}(A)=\rho(c(\sigma)) \sigma(A) \rho(c(\sigma))^{-1} \text {. }
$$

Let $e_{W} \in \operatorname{End}(W)$ be the identity. Since $W$ is defined over $F, \sigma\left(e_{W}\right)=e_{W}$ and similarly, $\tilde{\sigma}\left(e_{W}\right) e_{W}$. Thus $e_{W}$ is rational for the twisted action.

Remark 5.4. The representation $\rho$ is actually a representation of $\mathrm{G}^{s c}$. However, $\rho$ is irreducible and therefore maps $Z^{s c}$ to scalars. It follows that (5.1) is well-defined.

We use the twisted action to define an $F$-structure on $\operatorname{End}(W)$. We then claim that the map

is $F$-rational. Consider

$$
\mathrm{G} \ni g \mapsto \rho(g) \cdot e_{W}=\rho(g)
$$

$$
\begin{aligned}
\rho(c(\sigma) \sigma(g)) e_{W} & =\rho\left(c(\sigma) \sigma(g) c(\sigma)^{-1}\right) e_{W} \\
& =\rho(c(\sigma)) \rho(\sigma(g)) e_{W} \rho(c(\sigma))^{-1} \\
& =\rho(c(\sigma)) \sigma\left(\rho(g) e_{W}\right) \rho(c(\sigma))^{-1} .
\end{aligned}
$$

The latter equality holds since $e_{W}$ is $F$-rational. Thus

$$
\rho(c(\sigma) \sigma(g)) e_{W}=\tilde{\sigma}\left(\rho(g) e_{W}\right),
$$

which proves the assertion.

For $g \in \mathrm{G}$, let $\bar{\rho}(g)$ be the image of $\rho(g)$ in $\mathbb{P}(W)$. Then

$$
\bar{\rho}: \mathrm{G} \rightarrow \mathbb{P}(\operatorname{End}(W))
$$


is also $F$-rational (equivalently, $\bar{\rho}$ is $\Gamma$-equivariant for the twisted action). Thus $\bar{\rho}(\mathrm{G})$ and its closure $X$ in $\mathbb{P}(\operatorname{End}(W))$ are $\Gamma$-stable. This gives a canonical $F$-structure for the variety $X$ and on

$$
Y:=\bigcap_{\alpha \in \Delta} D_{\alpha}
$$

(the $\mathrm{G} \times \mathrm{G}$-orbit through the image of $v_{2 \lambda} \otimes v_{2 \lambda}^{*}$ in $\mathbb{P}\left(\operatorname{End}(W)\right.$ ), where $v_{2 \lambda}$ is "the" highest weight in $V_{2 \lambda}$; see 5.1 of [16]). Moreover, we have an equivariant isomorphism of $F$-varieties

$$
Y \simeq \mathrm{G} / \mathrm{B} \times \mathrm{G} / \mathrm{B}^{-} .
$$

Indeed, the $\bar{F}$-irreducible boundary components $D_{\alpha}$ of $X \backslash \mathrm{G}$ are permuted by $\Gamma$. Each component $D_{\alpha}$ is $\mathrm{G} \times \mathrm{G}$-stable and in particular stable for the action of the diagonal $\operatorname{Diag}(G)$ :

$$
A \mapsto \rho(g) A \rho^{-1}(g)
$$

From (5.1) we have

$$
\tilde{\sigma}\left(D_{\alpha}\right)=\sigma\left(D_{\alpha}\right)
$$

Thus $Y$ is invariant for the standard action.

We now prove that the $\mathrm{G} \times \mathrm{G}$-action on $X$ is $F$-rational for the twisted $F$ structure. For this we need to see that for $x \in X$,

$$
\tilde{\sigma}\left(\left(g_{1}, g_{2}\right)\right) x=\tilde{\sigma}\left(g_{1}, g_{2}\right) \tilde{\sigma}(x) .
$$

In fact, this holds for all $y \in \mathbb{P}(\operatorname{End}(W))$. We have to show that

$$
\tilde{\sigma}\left(\rho\left(g_{1}\right) A \rho\left(g_{2}\right)^{-1}\right)=\rho\left(\tilde{\sigma}\left(g_{1}\right)\right) \tilde{\sigma}(A) \rho\left(\tilde{\sigma}\left(g_{2}\right)^{-1}\right) .
$$

The left side is

$$
\rho(c(\sigma)) \sigma\left(\rho\left(g_{1}\right) A \rho\left(g_{2}\right)^{-1}\right) \rho(c(\sigma))^{-1},
$$

and the right side

$$
\begin{gathered}
\rho(c(\sigma)) \rho\left(\sigma\left(g_{1}\right)\right) \rho(c(\sigma))^{-1} \rho c(\sigma)(A) \rho(c(\sigma))^{-1} \rho(c(\sigma)) \rho\left(\sigma\left(g_{2}\right)\right)^{-1} \rho(c(\sigma))^{-1} \\
=\rho(c(\sigma)) \rho\left(\sigma\left(g_{1}\right)\right) \sigma(A) \rho\left(\sigma\left(g_{2}\right)\right)^{-1} \rho(c(\sigma))^{-1} .
\end{gathered}
$$

The assertion follows, since $\sigma$ and $\rho(g), g \in \mathrm{G}$, commute.

The restriction of line bundles to the unique closed $\mathrm{G} \times \mathrm{G}$-orbit $Y$ induces an injection

$$
\operatorname{Pic}(X) \rightarrow \operatorname{Pic}(Y) .
$$

Note that since each divisor $D_{\alpha}$ is $\mathrm{G} \times \mathrm{G}$-stable, the two actions of $\Gamma$ on $X$ give rise to the same action on $\operatorname{Pic}(X)$. Moreover, since $Y$ is defined over $F$, the injection (5.2) is $\Gamma$-equivariant. Here

$$
\operatorname{Pic}(Y) \simeq \mathfrak{X}\left(\mathrm{T}^{\prime}\right) \oplus \mathfrak{X}\left(\mathrm{T}^{\prime}\right)
$$

as $\Gamma$-modules. The image of $\operatorname{Pic}(X)$ is exactly the set of pairs $(\lambda,-\lambda)$, where $\lambda \in$ $\mathfrak{X}^{*}\left(\mathrm{~T}^{\prime}\right)$, with boundary divisors $D_{\alpha}$ corresponding to $(\alpha,-\alpha)$. The $F$-irreducible boundary components are divisors

$$
D_{J}=\sum_{\alpha \in J} D_{\alpha},
$$

for any $\Gamma$-stable subset $J \subset \Delta\left(\mathrm{G}^{\prime}, \mathrm{T}^{\prime}\right)$. 


\section{Heights}

6.1. Metrizations. Here we recall the definitions of (adelically) metrized line bundles and the associated heights.

Definition 6.1. Let $X$ be a smooth projective algebraic variety over a number field $F$. A smooth adelic metrization of a line bundle $L$ on $X$ is a family of $v$-adic norms $\|\cdot\|_{v}$ on $L \otimes_{F} F_{v}$ for all $v \in \operatorname{Val}(F)$ such that

- for $v \in S_{\infty}$ one has $\|\cdot\|_{v}$ is $C^{\infty}$;

- for $v \notin S_{\infty}$ the norm of any local section of $L$ is locally constant in $v$-adic topology;

- there exist a finite set $S \subset \operatorname{Val}(F)$, a flat projective scheme (an integral model) $\mathcal{X}$ over $\operatorname{Spec}\left(\mathcal{O}_{S}\right)$ with generic fiber $X$ together with a line bundle $\mathcal{L}$ on $\mathcal{X}$ such that for all $v \notin S$ the $v$-adic metric is given by the integral model.

Example 6.2. If $L$ is generated by global sections $\left(\mathrm{s}_{i}\right)$ and $\mathrm{s}$ is a section such that $\mathrm{s}(x) \neq 0$, then

$$
\|\mathrm{s}(x)\|_{v}:=\max _{i}\left(\left|\frac{\mathrm{s}_{i}}{\mathrm{~s}}(x)\right|_{v}\right)^{-1} .
$$

This defines a $v$-adic metric on $L$, which, of course, depends on the choice of the basis $\left(\mathrm{s}_{i}\right)$. An adelic metric on $L$ is a collection of $v$-adic metrics (for all $v$ ) such that there exists an $F$-rational basis $\left(\mathrm{s}_{j}\right)$ of $H^{0}(X, L)$ with the property that for all but finitely many $v$ the $v$-adic metric on $L$ is defined by means of this basis.

An adelically metrized line bundle $\mathcal{L}$ induces local and global heights: for any local section $\mathrm{s}$ of $L$ and any $x$ with $\mathrm{s}(x) \neq 0$ define

$$
H_{\mathrm{s}, \mathcal{L}, v}(x)=\|\mathbf{s}(x)\|_{v}^{-1} .
$$

For $x \in X(F)$ the product formula ensures that the global height

$$
H_{\mathcal{L}}(x)=\prod_{v \in \operatorname{Val}(F)} H_{\mathrm{s}, \mathcal{L}, v}(x)
$$

is independent of the choice of s. We write $\mathcal{L}=(L,\|\cdot\|)$ when we want to emphasize that $L \in \operatorname{Pic}(X)$ is adelically metrized.

Let $F$ be a number field and $V$ a finite-dimensional vector space over $F$. Thus $V$ is the set of $F$-rational points $V(F)$ of a linear variety $V$ defined over $F$. For $v \in \operatorname{Val}(F)$ we set $V_{v}:=V \otimes_{F} F_{v}$.

Suppose first that $v$ is non-archimedean. Let $\Lambda_{v}$ be an $\mathcal{O}_{v}$ lattice in $V_{v}$. We define the norm

$$
\|\cdot\|_{\Lambda_{v}}=\|\cdot\|_{v}
$$

on $V_{v}$ (associated to $\Lambda_{v}$ ) as follows. Let $n=\operatorname{dim}(V)$ and let

$$
\mathcal{B}_{v}:=\left\{\xi_{v}^{(1)}, \ldots, \xi_{v}^{(n)}\right\}
$$

be an $\mathcal{O}_{v}$-module basis of $\Lambda_{v}$. Let $v \in V_{v}$ have the form

$$
v=\sum_{j=1}^{n} a_{j} \xi_{v}^{(j)}
$$

We set

$$
\|v\|_{\Lambda_{v}}=\max _{1 \leq j \leq n}\left(\left|a_{j}\right|_{v}\right) .
$$


We see at once that $\|\cdot\|_{\Lambda_{v}}$ depends only on $\Lambda_{v}$ and not on the particular choice of a basis of $\mathcal{B}_{v}$.

Now suppose that $v$ is archimedean and let

$$
\mathcal{B}_{v}:=\left\{\xi_{v}^{(1)}, \ldots, \xi_{v}^{(n)}\right\}
$$

be a basis of the $F_{v}$-vector space $V_{v}$. For

$$
v=\sum_{j=1}^{n} a_{j} \xi_{v}^{(j)}
$$

we set

$$
\|v\|_{\Lambda_{v}}=\left(\sum_{1 \leq j \leq n}\left|a_{j}\right|_{v}^{2}\right)^{1 / 2}
$$

(for $v$ complex, we set $|z|_{v}^{2}=z \cdot \bar{z}$ ).

6.2. Heights on the canonical compactification. First we describe the situation for $\mathrm{G}^{s p}$. Recall that the (classes of) irreducible boundary components $D_{\alpha}$, $\alpha \in \Delta=\Delta\left(\mathrm{G}^{s p}, \mathrm{~T}^{s p}\right)$, generate $\operatorname{Pic}(X)$. Each divisor class $\left[D_{\alpha}\right]$ has a unique $\mathrm{G}^{s p} \times \mathrm{G}^{s p}$-stable representative - namely this component. We put $L_{\alpha}=\mathcal{O}_{X}\left(D_{\alpha}\right)$. This gives us a canonical splitting of the projection

$$
\operatorname{Div}(X) \rightarrow \operatorname{Pic}(X)
$$

which allows us to identify $\operatorname{Pic}(X)$ with the set of all integral linear combinations

$$
L=\sum_{\alpha \in \Delta} s_{\alpha} L_{\alpha},
$$

and $\operatorname{Pic}(X)_{\mathbb{C}}$ with formal sums $\sum_{\alpha \in \Delta} s_{\alpha} L_{\alpha}$.

We fix an integral model $\mathcal{L}_{\alpha}$ on the line bundle $L_{\alpha}$, for $\alpha \in \Delta$. If $\mathcal{L}_{\alpha}^{\prime}$ is another model, then the induced integral structures on $\mathcal{L}_{\alpha, v}$, resp. $\mathcal{L}_{\alpha, v}^{\prime}$, coincide for almost all $v$. An integral model defines a height function

$$
H_{\alpha}=H_{\mathcal{L}_{\alpha}}: X(F) \rightarrow \mathbb{R}_{>0}
$$

as in Section 6.1. Given an $L=\sum_{\alpha \in \Delta} s_{\alpha} L_{\alpha}$, with $s_{\alpha} \in \mathbb{C}$, we may define a height

$$
\begin{array}{rlc}
H_{L}: X(F) & \rightarrow \mathbb{C}, \\
x & \mapsto \prod_{\alpha} H_{\alpha}(x)^{s_{\alpha}} .
\end{array}
$$

We make the above construction more precise and explicit, by defining local heights

$$
H_{\alpha, v}: \mathrm{G}\left(F_{v}\right) \rightarrow \mathbb{R}_{>0}, \quad \text { for all } \alpha .
$$

For each $L_{\alpha}$ we fix the (unique, up to scalars) $F$-rational global section $\mathrm{s}_{\alpha} \in$ $H^{0}\left(X, L_{\alpha}\right)$, which is $\mathrm{G}^{s p} \times \mathrm{G}^{s p}$-invariant and non-vanishing on $\mathrm{G}^{s p}$. Using the integral structure, put, for $g_{v} \in \mathrm{G}^{s p}\left(F_{v}\right)$ and $\alpha \in \Delta$,

$$
H_{\alpha, v}\left(g_{v}\right):=\left\|\mathbf{s}_{\alpha}\left(g_{v}\right)\right\|_{v}^{-1} \text { and } H_{\alpha}:=\prod_{v} H_{\alpha, v} .
$$

Proposition 6.3. There is a lattice $\Lambda$ in some representation of $\mathrm{G}^{s p}$ with the following property: Let $\mathrm{K}_{f}$ be the stabilizer of $\Lambda_{f}$ in $\mathrm{G}^{s p}\left(\mathbb{A}_{f}\right)$. Then for almost all finite $v \in \operatorname{Val}(F)$ and for every

$$
L=\sum_{\alpha \in \Delta} n_{\alpha} L_{\alpha} \in \operatorname{Pic}(X), \quad \text { with } n_{\alpha} \geq 0,
$$


we have

$$
H_{L, v}\left(k_{1} g k_{2}\right)=H_{L, v}(g), \quad \text { for all } g \in \mathrm{G}\left(F_{v}\right), \quad k_{1}, k_{2} \in \mathrm{K}_{v} .
$$

Proof. If suffices to consider $L=L_{\lambda}$, for regular dominant weights $\lambda$. We may assume that the action of $\mathrm{G}^{s p} \times \mathrm{G}^{s p}$ on $L$ is defined over $F$ (passing to a multiple of $L$; see [28, Section 3, Prop. 1.5). Fix a lattice $\Lambda \subset H^{0}(X, L)_{F}$; it defines an $\mathcal{O}_{F}$-integral structure on $L$.

6.3. Local heights. Let $L=L_{\lambda}$ be a very ample $F$-rational line bundle on $X$ and fix a lattice $\Lambda \subset H^{0}(X, L)$. Over the (fixed) splitting field $E$, there is a distinguished $\mathrm{s} \in H^{0}(X, L)$ which is $\mathrm{G}^{s c} \times \mathrm{G}^{s c}$-invariant and non-vanishing on $\mathrm{G}(E)$. Moreover, $\mathrm{s}$ can be written as a product of sections $\mathrm{s}_{\alpha} \in H^{0}\left(X, L_{\alpha}\right), \alpha \in \Delta$, with support in the $E$-rational divisor in $D_{\alpha}$. Thus, for $\sigma \in \Gamma=\Gamma_{E / F}$ we have

$$
\sigma(\mathrm{s})=c(\sigma) \cdot \mathbf{s}
$$

for some $c(\sigma) \in E^{\times}$. By Hilbert's theorem 90, we may assume, after replacing s by a suitable multiple, that $\mathrm{s}$ is fixed by $\Gamma$, i.e., $\mathrm{s}$ is $F$-rational.

We have an $\mathcal{O}_{E}$-integral structure on $L$ over $E$, induced from $\Lambda \otimes_{\mathcal{O}_{F}} \mathcal{O}_{E}$. Over $E$, we have a decomposition

$$
H^{0}\left(X, L_{\lambda}\right)=\bigoplus_{\gamma}\left(V_{\gamma} \otimes V_{\gamma}^{*}\right)
$$

as $\mathrm{G}^{s c} \times \mathrm{G}^{s c}$-modules. Here the sum is over dominant $\gamma$ of the form $\gamma=\lambda-$ $\sum_{\alpha \in \Delta} m_{\alpha} \alpha$, with $m_{\alpha} \geq 0$. The following lemma is used to calculate the local height integrals.

Lemma 6.4. One may choose $S_{F}$ large enough, so that for $v \notin S_{F}$, if $g_{v}=k_{v} t_{v} k_{v}^{\prime}$ with $k_{v} \in \mathrm{K}_{v}$ and $t_{v} \in \mathrm{S}\left(F_{v}\right)^{+}$, then

$$
H_{v}\left(g_{v}\right)=\left|\chi_{\lambda}\left(t_{v}\right)\right|_{w} .
$$

Here $\chi_{\lambda}$ is the rational character of $\mathrm{T}$ associated with the dominant weight $\lambda$.

Proof. After replacing $L_{\lambda}$ by a positive integral multiple we can assume that $\lambda$ is trivial on the center of $\mathrm{G}^{s c}$. In particular, $\chi_{\lambda}\left(t_{v}\right) \in F_{v}^{\times}$. Since $L_{\lambda}$ is $\mathrm{G} \times \mathrm{G}$ linearizable, we have an $F$-rational representation $\varrho$ of $\mathrm{G} \times \mathrm{G}$ on $H^{0}\left(X, L_{\lambda}\right)$. Then for $g_{v} \in \mathrm{G}\left(F_{v}\right)$ we have

where $\Lambda_{v}=\Lambda \otimes_{\mathcal{O}_{F}} \mathcal{O}_{v}$.

$$
H_{v}\left(g_{v}\right)=\left\|\varrho\left(g_{v}\right)\right\|_{\Lambda_{v}}
$$

Let $\Lambda_{E} \subset H^{0}(X, L)_{E}=V_{E}$ be an "admissible" lattice, i.e., $\Lambda_{E}$ is homogeneous with respect to the decomposition of $V=\bigoplus_{\gamma} V_{\gamma}$ into weight spaces. More precisely, the representation of $\mathrm{G}^{s p} \times \mathrm{G}^{s p}$ on $V$ has a $\mathbb{Z}$-form on $\Lambda_{\mathbb{Z}}$ with $\Lambda_{\mathbb{Z}}$ a homogeneous lattice in $V_{\mathbb{Q}}$. We set $\Lambda_{\mathcal{O}_{E}}:=\Lambda_{\mathbb{Z}} \otimes_{\mathbb{Z}} \mathcal{O}_{E}$ and $\Lambda_{\mathcal{O}_{F}}:=\Lambda_{\mathcal{O}_{E}} \cap V_{F}$. Let $\mathcal{G}$ be the group scheme structure on the double stabilizer of $\Lambda_{\mathcal{O}_{F}}$ and let $\mathrm{K}_{v}=\mathcal{G}\left(\mathcal{O}_{v}\right), \mathrm{K}_{w}=\mathcal{G}\left(\mathcal{O}_{w}\right)$. We choose $S_{F}$ so that for all $v \notin S_{F}$ and $w \mid v$

$$
\Lambda_{w}=\Lambda_{v} \otimes_{\mathcal{O}_{v}} \mathcal{O}_{w} .
$$

We choose $|\cdot|_{w}$ so that its restriction to $F_{v}$ coincides with $|\cdot|_{v}$. Then the restriction of $\|\cdot\|_{\Lambda_{w}}$ to $V_{v}$ is $\|\cdot\|_{\Lambda_{v}}$ and, for $g \in \mathrm{G}\left(F_{v}\right)$

$$
H_{v}\left(g_{v}\right)=\left\|\varrho\left(g_{v}\right)\right\|_{\Lambda_{w}} .
$$

Write, according to Corollary [1.3, that

$$
g_{v}=a_{w} x_{v} a_{w}^{-1}
$$


with $x_{v} \in \mathrm{G}^{\prime}\left(F_{v}\right), a_{w} \in \mathcal{G}\left(\mathcal{O}_{w}\right)=\mathrm{K}_{w}$. Then

$$
H_{v}\left(g_{v}\right)=\left\|\varrho\left(a_{w}\right) \varrho\left(x_{v}\right) \varrho\left(a_{w}\right)^{-1}\right\|_{\Lambda_{w}}=\left\|\varrho\left(x_{v}\right)\right\|_{\Lambda_{w}} .
$$

We next set

$$
\mathrm{K}_{v}^{\prime}:=\mathrm{K}_{w} \cap \mathrm{G}^{\prime}\left(F_{v}\right) .
$$

Recall that $\mathrm{G}\left(F_{v}\right) \subset \mathrm{G}\left(E_{w}\right), \mathrm{G}^{\prime}\left(F_{v}\right) \subset \mathrm{G}\left(E_{w}\right)$. By Bruhat-Tits theory, we have

$$
\mathrm{G}^{\prime}\left(F_{v}\right)=\mathrm{K}_{v}^{\prime} \mathrm{S}^{\prime}\left(F_{v}\right)^{+} \mathrm{K}_{v}^{\prime} \text {. }
$$

Write accordingly, $x_{v}=k_{1} t_{v} k_{2}$, with $k_{1}, k_{2} \in \mathrm{K}_{v}^{\prime}, t_{v} \in \mathrm{S}^{\prime}\left(F_{v}\right)^{+}$. Then

$$
H_{v}\left(g_{v}\right)=\left\|\varrho\left(t_{v}\right)\right\|_{\Lambda_{w}} .
$$

Next choose a basis $\mathcal{B}=\left\{v_{\mu}\right\}$ of $\Lambda_{w}$ consisting of weight vectors for $\mathrm{T}_{v}^{s p}$. Thus

$$
H_{v}\left(g_{v}\right)=\max _{\mu}\left|\mu\left(t_{v}\right)\right|_{w} .
$$

Each $\mu$ has the form

$$
\mu=\chi_{\lambda} \cdot \prod_{\alpha \in \Delta} \alpha^{-m_{\alpha}}
$$

with $m_{\alpha} \in \mathbb{N}$. Since $t_{v} \in \mathrm{S}_{v}^{\prime}\left(F_{v}\right)^{+}$, we have $\left|\alpha\left(t_{v}\right)\right|_{w}=\left|\alpha\left(t_{v}\right)\right|_{v} \geq 1$. Hence finally

$$
H_{v}\left(g_{v}\right)=\left|\chi_{\lambda}\left(t_{v}\right)\right|_{v}
$$

6.4. Local integrals representing heights. Let $D$ be a central simple algebra of rank $m$ over the number field $F$. Also let $\Lambda$ be an arbitrary lattice in D. We set, for each place $v, \mathrm{D}_{v}=\mathrm{D} \otimes_{F} F_{v}$, and if $v$ is non-archimedean, $\Lambda_{v}=\Lambda \otimes_{\mathcal{O}_{F}} \mathcal{O}_{v}$. In this subsection, we define a family of norms $\|\cdot\|_{\Lambda_{v}}$ on $\mathrm{D}_{v}$, one for each place $v$ of $F$, subject to a certain compatibility condition.

- non-archimedean $v$ : Choose a basis $\left\{\xi_{1}^{v}, \ldots, \xi_{k}^{v}\right\}$ for $\mathrm{D}_{v}$ with $\xi_{i}^{v} \in \Lambda_{v}=$ $\Lambda \otimes_{\mathcal{O}} \mathcal{O}_{v}$ for all $i$. For $g \in \mathrm{D}_{v}$, write $g=\sum_{i} c_{i}(g) \xi_{i}^{v}$ and set

$$
\|g\|_{v}=\|g\|_{\Lambda_{v}}:=\max _{i=1, \ldots, k}\left\{\left|c_{i}(g)\right|_{v}\right\} .
$$

It is easy to see that this norm is right and left $\Lambda_{v}^{\times}$-invariant and therefore independent of the choice of the basis.

- archimedean v: Fix a Banach space norm $\|\cdot\|_{v}=\|\cdot\|_{\mathrm{D}_{v}}$ on the finitedimensional real (or complex) vector space $\mathrm{D}_{v}=\mathrm{D} \otimes_{F} F_{v}$.

Clearly, for $c \in F_{v}$ and $g \in \mathrm{D}_{v}$, we have

$$
\|c g\|_{v}=|c|_{v} \cdot\|g\|_{v} .
$$

Consequently, for $c \in F$ and $g \in D$, we have

$$
\prod_{v}\|c g\|_{v}=\prod_{v}\|g\|_{v}
$$

by the product formula. This is the compatibility condition mentioned above. Define a function $\Psi_{v}$ as follows:

$$
\Psi_{v}= \begin{cases}\text { characteristic function of } \Lambda_{v}, & \text { for } v \notin S_{\infty}, \\ \exp \left(-\pi\|\cdot\|_{v}^{2}\right) & \text { for } v \in S_{\infty} .\end{cases}
$$


Lemma 6.5. For all $v \in \operatorname{Val}(F)$, all $g_{v} \in \mathrm{D}_{v}$ and all $s$ with $\Re(s)>0$ one has

$$
\int_{F_{v}^{\times}} \Psi_{v}\left(a g_{v}\right)|a|^{s} d^{\times} a=\zeta_{F, v}(s) \cdot\left\|g_{v}\right\|_{v}^{-s} .
$$

Here $\zeta_{F, v}(s)$ is the v-local Euler factor of the zeta function of $F$.

For $\Phi_{v} \in C_{c}^{\infty}\left(\mathrm{D}_{v}\right)$ and $\Re(s)>0$ we set

$$
H_{v}\left(s, g ; \Phi_{v}\right):=\zeta_{F, v}(s)^{-1} \int_{F_{v}^{\times}} \Phi_{v}(a g)|a|^{s} d^{\times} a,
$$

where $g \in \mathrm{D}_{v}$. For $g=\left(g_{v}\right)_{v} \in \mathrm{D}(\mathbb{A})$ define the global height function:

$$
H(g)=\prod_{v \in \operatorname{Val}(F)} H_{v}(g)=\prod_{v \in \operatorname{Val}(F)}\left\|g_{v}\right\|_{v} .
$$

Similarly, if $\Phi=\bigotimes_{v} \Phi_{v}$ is a global Schwartz-Bruhat function on $\mathrm{D}(\mathbb{A})$, we define

$$
H(s, g ; \Phi)=\prod_{v \in \operatorname{Val}(F)} H_{v}\left(s, g_{v} ; \Phi_{v}\right) .
$$

We extend the functional $H(s, g ; \cdot)$ to $C_{c}^{\infty}(\mathrm{D}(\mathbb{A}))$ by linearity. By the product formula, both $H(g)$ and $H(s, g ; \Phi)$ (for $\Phi \in C_{c}^{\infty}(\mathrm{D}(\mathbb{A}))$ ) are well-defined on the projective group of $\mathrm{D}$.

\subsection{Complexified height function.}

Notation 6.6. Let $\mathcal{T}$ be the set of all Galois invariants $\mathbf{s}=\left(s_{\alpha}\right)_{\alpha \in \Delta\left(\mathrm{G}^{\prime}, \mathrm{T}^{\prime}\right)}$ (cf. Section 2.9 for the definition of Galois invariance). The element $\underline{0} \in \mathcal{T}$ is defined by setting all coordinates equal to zero. For $\epsilon \in \mathbb{R}$, the set $\mathcal{T}_{\epsilon}$ is the set of $\mathbf{s}=\left(s_{\alpha}\right)_{\alpha} \in \mathcal{T}$ such that $\Re\left(s_{\alpha}\right)>\kappa_{\alpha}+1+\epsilon$, for all $\alpha$. Starting with an element $\mathbf{s}=\left(s_{\alpha}\right)_{\alpha \in \Delta\left(\mathrm{G}^{\prime}, \mathrm{T}^{\prime}\right)}$ and $v \notin S$, we obtain a tuple $\mathbf{s}^{v}=\left(s_{\vartheta}^{v}\right)$ indexed by $\Delta\left(\mathrm{G}^{\prime}\left(F_{v}\right), \mathrm{S}_{v}^{\prime}\left(F_{v}\right)\right)$ by setting $s_{r_{v}\left(\iota^{*}(\alpha)\right)}^{v}=s_{\alpha}$; this is well-defined. For $\mathbf{s}, \mathbf{t} \in \mathcal{T}$ and $v \notin S$, we set

$$
\langle\mathbf{s}, \mathbf{t}\rangle_{v}=\sum_{\vartheta \in \Delta\left(\mathrm{G}^{\prime}\left(F_{v}\right), \mathrm{S}_{v}^{\prime}\left(F_{v}\right)\right)} s_{\vartheta}^{v} t_{\vartheta}^{v} .
$$

When there is no danger of confusion, we write $\mathbf{s}$ for $\mathbf{s}^{v}$. For each subset $R$ of $\mathbb{C}$, we set $\mathcal{T}(R)$ to be the collection of $\mathbf{s}=\left(s_{\alpha}\right)_{\alpha}$ with $s_{\alpha} \in R$ for all $\alpha$.

We go back to Lemma 6.4. For $v \notin S_{F}$, we have expressed $H_{v}(g)$ in terms of $\chi_{\lambda} \in \mathfrak{X}^{*}(\mathrm{~T})$. Write

$$
\chi_{\lambda}=\prod_{\alpha \in \Delta\left(\mathrm{G}^{\prime}, \mathrm{T}^{\prime}\right)} \alpha^{n_{\alpha}}
$$

and $\lambda=\sum_{\alpha \in \Delta(\mathrm{G}, \mathrm{T})} n_{\alpha} \alpha, n_{\alpha} \in \mathbb{N}$. We know that $\left(n_{\alpha}\right)_{\alpha \in \Delta\left(\mathrm{G}^{\prime}, \mathrm{T}^{\prime}\right)} \in \mathcal{T}$. Now, let $\mathbf{s}:=\left(s_{\alpha}\right)_{\alpha \in \Delta\left(\mathrm{G}^{\prime}, \mathrm{T}^{\prime}\right)} \in \mathcal{T}$. Suppose $v \notin S_{F}$, and $g_{v} \in \mathrm{G}^{\prime}\left(F_{v}\right)$ is written as $k_{v} t_{v} k_{v}^{\prime}$ with $t_{v} \in \mathrm{S}_{v}^{\prime}\left(F_{v}\right)^{+}$and $k_{v}, k_{v}^{\prime} \in \mathrm{K}_{v}^{\prime}$. We define

$$
H_{v}\left(\mathbf{s}, g_{v}\right)=\prod_{\alpha \in \Delta\left(\mathrm{G}^{\prime}, \mathrm{T}^{\prime}\right)}\left|\alpha\left(t_{v}\right)\right|_{v}^{s_{\alpha}} .
$$

Observe that

$$
H_{v}\left(\mathbf{s}, g_{v}\right)=\prod_{\vartheta \in \Delta\left(\mathrm{G}^{\prime}\left(F_{v}\right), \mathrm{S}_{v}^{\prime}\left(F_{v}\right)\right)}\left|\vartheta\left(t_{v}\right)\right|_{v}^{\ell_{v}(\vartheta) s_{\vartheta}} .
$$


If we use Corollary 1.3 to identify the groups $\mathrm{G}\left(F_{v}\right)$ and $\mathrm{G}^{\prime}\left(F_{v}\right)$, we get a complexified local height function on $\mathrm{G}\left(F_{v}\right)$. Let $S=S_{F}$ and suppose $g=\left(g_{v}\right)_{v \notin S} \in \mathrm{G}\left(\mathbb{A}_{S}\right)$. We define

$$
H_{S}(\mathbf{s}, g):=\prod_{v \notin S} H_{v}\left(\mathbf{s}, g_{v}\right)
$$

6.6. Local height integrals I. Consider the integral of the complexified local height function

$$
\mathcal{J}_{v}(\mathbf{s}):=\int_{\mathrm{G}_{v}} H_{v}(\mathbf{s}, g)^{-1} d g .
$$

Theorem 6.7. (1) For all $v \notin S_{\infty}$ the integral $\mathcal{J}_{v}(\mathbf{s})$ is a holomorphic function of $\mathbf{s}$ for $\mathbf{s} \in \mathcal{T}_{-1}$.

(2) Let $v$ be an archimedean valuation and $\partial$ any element of the universal enveloping algebra. Then

$$
\mathcal{J}_{v, \partial}(\mathbf{s}):=\int_{\mathbf{G}\left(F_{v}\right)} \partial\left(H(\mathbf{s}, g)^{-1}\right) d g
$$

is holomorphic for $\mathbf{s} \in \mathcal{T}_{-1}$.

Proof. We will only prove the first part; the second part is similar. Locally, every two local integral structures give rise to essentially equivalent height functions; so, we replace the local integral structure so that the resulting height function is invariant under $\mathrm{K}_{v}$, a good maximal compact subgroup. Let $\underline{\sigma}$ be the vector consisting of the real parts of the components of $\mathbf{s}$. The local height integral is majorized by

$$
\begin{aligned}
& \sum_{t \in \mathrm{S}\left(F_{v}\right)^{+}} \sum_{\omega \in \Omega_{v}} H(\underline{\sigma}, t \omega)^{-1} \operatorname{vol}\left(\mathrm{K}_{v} t \omega \mathrm{K}_{v}\right) \\
\ll & \sum_{t \in \mathrm{S}\left(F_{v}\right)^{+}} H(\underline{\sigma}, t)^{-1} \delta_{B}(t) \\
= & \prod_{\vartheta \in \Delta\left(\mathrm{G}\left(F_{v}\right), \mathrm{S}_{v}\left(F_{v}\right)\right)} \sum_{l=0}^{\infty} \delta_{B}\left(\check{\vartheta}\left(\varpi_{v}^{l}\right)\right) H\left(\underline{\sigma}, \check{\vartheta}\left(\varpi_{v}^{l}\right)\right)^{-1} \\
= & \prod_{\vartheta \in \Delta\left(\mathrm{G}\left(F_{v}\right), \mathrm{S}_{v}\left(F_{v}\right)\right)} \sum_{l=0}^{\infty} q_{v}^{-\left(\sigma_{\vartheta}-\kappa_{\vartheta}\right) l \ell(\vartheta)} .
\end{aligned}
$$

The result is now immediate.

Corollary 6.8. In the non-archimedean situation, for each $\epsilon>0$ there is a constant $C_{v}(\epsilon)$, such that $\left|\mathcal{J}_{v}(\mathbf{s})\right| \leq C_{v}(\epsilon)$ for all $\mathbf{s} \in \mathcal{T}_{-1+\epsilon}$. In the archimedean situation, for all $\epsilon>0$ and all $\partial$ as above, there is a constant $C_{v}(\partial, \epsilon)$ such that $\left|\mathcal{J}_{v, \partial}(\mathbf{s})\right| \leq$ $C_{v}(\partial, \epsilon)$ for all $\mathbf{s} \in \mathcal{T}_{-1+\epsilon}$. 
6.7. The integral of the local height function II. Let $G$ be a connected algebraic group over $F_{v}, v$ outside a finite set of places, and $\mathfrak{g}$ its Lie algebra of invariant vector fields. Let $X$ be a smooth equivariant compactification of G. Denote by $D=X \backslash \mathrm{G}$ the boundary. We assume that $D$ is a divisor with strict normal crossings. Let $\mathcal{T}_{X}$ be the tangent bundle of $X$. We have a restriction map

$$
H^{0}\left(X, \mathcal{T}_{X}\right) \rightarrow \mathcal{T}_{X, 1}=\mathfrak{g}
$$

obtained by evaluating a vector field at the neutral element $1 \in \mathrm{G}$. Conversely, given $\partial \in \mathfrak{g}$, there is a unique vector field $\partial^{X}$ such that for any open subset $U$ of $X$ and for any $f \in \mathcal{O}_{X}(U), \partial^{X}(f)(x)=\left.\partial_{g} f(g \cdot x)\right|_{g=1}$. The map $\partial \mapsto \partial^{X}$ is a section of the restriction map. Let $\partial_{1}, \ldots, \partial_{n}$ be a basis for $\mathfrak{g}$. Then $\delta:=\partial_{1}^{X} \wedge \cdots \wedge \partial_{n}^{X}$ is a global section of the line bundle $\operatorname{det}\left(\mathcal{T}_{X}\right)=K_{X}^{-1}$. Moreover, $\delta$ does not vanish on $\mathrm{G}$. Because of these considerations, if we know that $K_{X}^{-1}$ is ample, Peyre's Tamagawa measure restricts to Weil's Tamagawa measure on $\mathrm{G}\left(F_{v}\right)$.

If $\mu_{v}$ is the local Weil-Tamagawa measure, for $v$ outside of a finite set of places, we have

$$
\mu_{v}\left(\mathrm{G}\left(\mathcal{O}_{v}\right)\right)=\frac{\# \mathrm{G}\left(\mathbf{k}_{v}\right)}{q_{v}^{\operatorname{dim}(G)}}
$$

Therefore, we normalize the local measure by the appropriate factor to guarantee that $\mu\left(\mathrm{G}\left(\mathcal{O}_{v}\right)\right)=1$ for almost all $v$.

Let $A$ be a subset of $\Delta(\mathrm{G}, \mathrm{T})$; we set

$$
D_{A}=\bigcap_{\alpha \in A} D_{\alpha}
$$

and

$$
D_{A}^{0}=D_{A} \backslash\left(\bigcup_{A \subsetneq A^{\prime}} D_{A^{\prime}}\right),
$$

for $D_{\alpha}$ as defined in Section 5.5. The following theorem is the analog of Theorem 9.1 of [15] in this situation, with the same proof (see also Theorem 3.1 of [18]).

Theorem 6.9. We have

$$
\int_{\mathrm{G}\left(F_{v}\right)} H_{v}\left(\mathrm{~s}, g_{v}\right)^{-1} d g_{v}=\frac{1}{\# \mathrm{G}\left(\mathbf{k}_{v}\right)} \sum_{A} \# D_{A}^{0}\left(\mathbf{k}_{v}\right) \prod_{\alpha \in A} \frac{q_{v}-1}{q_{v}^{s_{\alpha}-\kappa_{\alpha}+1}-1} .
$$

Proof. We split the integral along residue classes modulo $\mathfrak{p}_{v}$. Let $\tilde{x} \in X\left(\mathbf{k}_{v}\right), \mathbf{k}_{v}$ the residue field of $F_{v}$, and $A=\left\{\alpha \mid \tilde{x} \in D_{\alpha}\right\}$, so that $\tilde{x} \in D_{A}^{0}$.

We can introduce local (étale) coordinates $x_{\alpha}(\alpha \in A)$ and $y_{\beta}(\beta \in B)$ with $\# A+\# B=\operatorname{dim}(X)$ around $\tilde{x}$ such that, locally, the divisor $D_{\alpha}$ is defined by the vanishing of $x_{\alpha}$. Then the local Tamagawa measure identifies with the measure $\prod d x_{\alpha} \times \prod d y_{\beta}$ on $\mathfrak{p}_{v}^{A} \times \mathfrak{p}_{v}^{B}$. If $d x$ denotes the fixed measure on $\mathrm{G}\left(F_{v}\right)$, one has the equality of measures on $\mathrm{G}\left(F_{v}\right) \cap \operatorname{red}^{-1}(\tilde{x})$ :

$$
d x=\frac{q_{v}^{\operatorname{dim}(X)}}{\# \mathrm{G}\left(\mathbf{k}_{v}\right)} H_{v}(\rho, x) d \mu_{v}=\frac{q_{v}^{\operatorname{dim}(X)}}{\# \mathrm{G}\left(\mathbf{k}_{v}\right)} \prod_{\alpha \in A} q_{v}^{\left(\kappa_{\alpha}+1\right) v\left(x_{\alpha}\right)} \prod d x_{\alpha} \prod d y_{\beta} .
$$


Consequently,

$$
\begin{aligned}
\int_{r_{e d}-1(\tilde{x})} & H_{v}(\mathbf{s}, x)^{-1} d x \\
= & \frac{q_{v}^{\operatorname{dim}(X)}}{\# \mathrm{G}\left(\mathbf{k}_{v}\right)} \int_{\mathfrak{p}_{v}^{A} \times \mathfrak{p}_{v}^{B}} q_{v}^{-\sum_{\alpha \in A}\left(s_{\alpha}-\kappa_{\alpha}-1\right) v\left(x_{\alpha}\right)} \prod d x_{\alpha} \prod d y_{\beta} \\
= & \frac{q_{v}^{\operatorname{dim}(X)}}{q_{v}^{\# B} \# \mathrm{G}\left(\mathbf{k}_{v}\right)} \prod_{\alpha \in A} \int_{\mathfrak{p}_{v}} q_{v}^{-\left(s_{\alpha}-\kappa_{\alpha}-1\right) v\left(x_{\alpha}\right)} d x_{\alpha} \\
= & \frac{1}{\# \mathrm{G}\left(\mathbf{k}_{v}\right)} \prod_{\alpha \in A} \frac{q_{v}-1}{q_{v}^{s_{\alpha}-\kappa_{\alpha}}-1} .
\end{aligned}
$$

We have used the identity

$$
\begin{aligned}
\int_{\mathfrak{p}_{v}} q_{v}^{-s v(x)} d x & =\sum_{n=1}^{\infty} q_{v}^{-s n} \operatorname{vol}\left(\mathfrak{p}_{v}^{n} \backslash \mathfrak{p}_{v}^{n+1}\right) \\
& =\sum_{n=1}^{\infty} q_{v}^{-s n} q_{v}^{-n}\left(1-\frac{1}{q_{v}}\right) \\
& =\frac{1}{q_{v}} \frac{q_{v}-1}{q_{v}^{1+s}-1}
\end{aligned}
$$

6.8. An application to volumes. We start with the following lemma in the nonarchimedean situation:

Lemma 6.10. Let $\mathrm{K}_{v}$ be a good maximal compact subgroup of $\left(\mathrm{G}\left(F_{v}\right), \mathrm{S}\left(F_{v}\right)\right)$ so that the Cartan decomposition $\mathrm{G}=\mathrm{K}_{v} \mathrm{~S}\left(F_{v}\right)^{+} \Omega \mathrm{K}_{v}$ holds. Normalize measures so that $\operatorname{vol}\left(\mathrm{K}_{v}\right)=1$. Then for all ad $\in \mathrm{S}\left(F_{v}\right)^{+} \Omega$, we have

$$
\operatorname{vol}\left(\mathrm{K}_{v} a d \mathrm{~K}_{v}\right) \ll \delta_{\mathrm{B}}(a) .
$$

As $\Omega$ is a finite set, this is an immediate consequence of Lemma 4.1.1 of [39]. Below we need more detailed information on the behavior of the the above volume in the quasi-split situation. The following lemma suffices for our purposes; the analogous statement for simply-connected is classical. For split groups, the lemma is contained in 22 .

Lemma 6.11. There exists a constant $c$, independent of $v$, such that for all $t \in$ $\mathrm{S}\left(F_{v}\right)^{+}$, one has

$$
\operatorname{vol}\left(\mathrm{K}_{v} t \mathrm{~K}_{v}\right) \leq \delta_{\mathrm{B}}(t)\left(1+\frac{c}{q_{v}}\right) .
$$

Proof. We use Theorem 6.9. The left hand side of (6.9) is obviously equal to

$$
\sum_{t \in \mathrm{S}\left(F_{v}\right)^{+}} H(\mathrm{~s}, t)^{-1} \operatorname{vol}\left(\mathrm{K}_{v} t \mathrm{~K}_{v}\right) .
$$

The comparison of this expression with the right hand side of (6.9) will give an explicit formula for the volume from which our result will easily follow. Clearly the right hand side of (6.9) is equal to

$$
\sum_{t \in \mathrm{S}\left(F_{v}\right)^{+}} \frac{\# D_{A(t)}^{0}\left(\mathbf{k}_{v}\right)(q-1)^{\# A(t)}}{\# \mathrm{G}\left(\mathbf{k}_{v}\right)} \delta_{\mathrm{B}}(t) H(\mathrm{~s}, t)^{-1} .
$$


Here $A(t)=\{\delta \in \Delta(\mathrm{G}, \mathrm{S}) ; \delta(t)=1\}$; we will suppress the dependence on $t$ and simply write $A$. Comparison gives

$$
\operatorname{vol}\left(\mathrm{K}_{v} t \mathrm{~K}_{v}\right)=\frac{\# D_{A}^{0}\left(\mathbf{k}_{v}\right)(q-1)^{\# A(t)}}{\# \mathrm{G}\left(k_{v}\right)} \delta_{\mathrm{B}}(t) .
$$

The algebraic set $D_{A}$ is the fiber variety $D_{A} \rightarrow \mathrm{G} / \mathrm{P}_{A} \times \mathrm{G} / \mathrm{P}_{A}$, with fibers isomorphic to $\overline{\mathrm{M}}_{A}$, the wonderful compactification of the adjoint group of $\mathrm{M}_{A}$, i.e., $\mathrm{M}_{A}$ modulo its center. Here $\mathrm{M}_{A}$ is the Levi factor of the parabolic subgroup $\mathrm{P}_{A}$. Then $D_{A}^{0}$ has fibers $\mathrm{M}_{A}$ modulo its center. Consequently,

$$
\# D_{A}^{0}\left(\mathbf{k}_{v}\right)=(q-1)^{-\# A}\left[\mathrm{G}\left(\mathbf{k}_{v}\right): \mathrm{P}_{A}\left(\mathbf{k}_{v}\right)\right]^{2} \cdot \# \mathrm{M}_{A}\left(\mathbf{k}_{v}\right) .
$$

If $\mathrm{U}_{A}$ is the unipotent radical of $\mathrm{P}_{A}$, we have

$$
\frac{\# D_{A}^{0}\left(\mathbf{k}_{v}\right)(q-1)^{\# A}}{\# \mathrm{G}\left(\mathbf{k}_{v}\right)}=\left[\mathrm{G}\left(\mathbf{k}_{v}\right): \mathrm{P}_{A}\left(\mathbf{k}_{v}\right)\right]\left(\# \mathrm{U}_{A}\left(\mathbf{k}_{v}\right)\right)^{-1} .
$$

Let $\mathrm{W}_{A}$ be the Weyl group of the Levi factor $\mathrm{M}_{A}$. By the Bruhat decomposition we have

$$
\mathbf{G}\left(\mathbf{k}_{v}\right)=\bigcup_{w \in \mathrm{W}_{A} \backslash \mathrm{W} / \mathrm{W}_{A}} \mathrm{P}_{A}\left(\mathbf{k}_{v}\right) w\left(\mathbf{U}_{A}\left(\mathbf{k}_{v}\right) \cap w^{-1} \overline{\mathbf{U}}_{A}\left(\mathbf{k}_{v}\right) w\right),
$$

where $\overline{\mathrm{U}}_{A}$ the unipotent radical opposite to $\mathrm{U}_{A}$. It follows that

$$
\frac{\left[\mathrm{G}\left(\mathbf{k}_{v}\right): \mathrm{P}_{A}\left(\mathbf{k}_{v}\right)\right]}{\# \mathrm{U}_{A}\left(\mathbf{k}_{v}\right)}=\sum_{w \in \mathrm{W}_{A} \backslash \mathrm{W} / \mathrm{W}_{A}} \frac{\#\left(\mathrm{U}_{A}\left(\mathbf{k}_{v}\right) \cap w^{-1} \overline{\mathrm{U}}_{A}\left(\mathbf{k}_{v}\right) w\right)}{\# \mathrm{U}_{A}\left(\mathbf{k}_{v}\right)} .
$$

As there is only one double coset of maximal dimension, we have

$$
\frac{\left[\mathrm{G}\left(\mathbf{k}_{v}\right): \mathrm{P}_{A}\left(\mathbf{k}_{v}\right)\right]}{\# \mathrm{U}_{A}\left(\mathbf{k}_{v}\right)} \leq 1+\frac{\#\left(\mathrm{~W}_{A} \backslash \mathrm{W} / \mathrm{W}_{A}\right)}{q_{v}} .
$$

The lemma is now clear for split groups. In the quasi-split case, we need only consider $\Gamma$-stable subsets $A$. For the complex parameters $s_{\alpha}$ we have the extra assumption that $s_{\alpha}=s_{\sigma \alpha}$, for $\sigma \in \Gamma$. The proof of the lemma in the quasi-split case is similar, and we omit it.

\section{Regularization}

In this section, for $v \notin S_{F}$, with a (slight) abuse of notation we identify $\mathrm{G}\left(F_{v}\right)$ and $\mathrm{G}^{\prime}\left(F_{v}\right)$; this is permissible, in light of Corollary 1.3 .

7.1. Integrals from one-dimensional representations. Let $\chi$ be a one-dimensional automorphic representation of G, and $S=S_{F}$. We proceed to study analytic properties of the integral

$$
\mathcal{J}_{S}(\mathbf{s}, \chi)=\int_{\mathrm{G}\left(\mathbb{A}_{S}\right)} H_{S}(\mathbf{s}, g)^{-1} \chi(g) d g
$$

for Galois invariant $\mathbf{s}$.

Theorem 7.1. The product

$$
\prod_{\mathfrak{O}} L\left(s_{\mathfrak{O}}, \xi_{\mathfrak{O}}(\chi)\right)^{-1} \int_{\mathfrak{G}\left(\mathbb{A}_{S}\right)} H_{S}(\mathbf{s}, g)^{-1} \chi(g) d g
$$

is holomorphic on $\mathcal{T}_{-\epsilon}$ for any $\epsilon<\frac{1}{2}$. The product is over all Galois orbits in $\Delta\left(\mathrm{G}^{\prime}, \mathrm{T}^{\prime}\right)$. 
Proof. We have

$$
\mathcal{J}_{S}(\mathbf{s}, \chi)=\prod_{v \notin S} \mathcal{J}_{v}\left(\mathbf{s}, \chi_{v}\right)
$$

where

$$
\mathcal{J}_{v}\left(\mathbf{s}, \chi_{v}\right)=\int_{\mathrm{G}\left(F_{v}\right)} H_{v}\left(\mathbf{s}, g_{v}\right)^{-1} \chi_{v}\left(g_{v}\right) d g_{v}
$$

Since $\mathrm{G}$ is of adjoint type, the collection of elements $\left\{\check{\vartheta}\left(\varpi_{v}\right)\right\}_{\vartheta \in \Delta\left(G\left(F_{v}\right), S_{v}\left(F_{v}\right)\right)}$ forms a basis for the semigroup $\mathrm{S}_{v}\left(F_{v}\right)^{+}$. For any vector $\mathbf{a}=\left(a_{\alpha}\right)_{\alpha} \in \mathcal{T}(\mathbb{N})$, we set

$$
t_{v}(\mathbf{a})=\prod_{\vartheta \in \Delta\left(\mathrm{G}\left(F_{v}\right), \mathrm{S}_{v}\left(F_{v}\right)\right)} \check{\vartheta}\left(\varpi_{v}\right)^{a_{\vartheta}}
$$

Write

$$
1+a_{v}=\sum_{\mathbf{a} \in \mathcal{T}(\mathbb{N})} q_{v}^{-\langle\mathbf{a}, \mathbf{s}\rangle_{v}} \chi_{v}\left(t_{v}(\mathbf{a})\right) \delta\left(t_{v}(\mathbf{a})\right)
$$

and

$$
b_{v}=\sum_{\mathbf{a} \in \mathcal{T}(\mathbb{N})} q_{v}^{-\langle\mathbf{a}, \mathbf{s}\rangle_{v}} \chi_{v}\left(t_{v}(\mathbf{a})\right)\left(\operatorname{vol}\left(\mathrm{K}_{v} t_{v}(\mathbf{a}) \mathrm{K}_{v}\right)-\delta\left(t_{v}(\mathbf{a})\right)\right)
$$

so that, by Cartan Decomposition, we have

$$
\mathcal{J}_{v}\left(\mathbf{s}, \chi_{v}\right)=1+a_{v}+b_{v} .
$$

Observe that

$$
\begin{aligned}
1+a_{v} & =\sum_{\mathbf{a}} q_{v}^{-\langle\mathbf{a}, \mathbf{s}\rangle_{v}} \chi_{v}\left(t_{v}(\mathbf{a})\right) \delta\left(t_{v}(\mathbf{a})\right) \\
& =\prod_{\vartheta}\left(\sum_{a_{\vartheta}=0}^{\infty} \chi_{v}\left(\check{\vartheta}\left(\varpi_{v}\right)\right)^{a_{\vartheta}} q_{v}^{-\left(s_{\vartheta}-\kappa_{\vartheta}\right) a_{\vartheta} \ell(\vartheta)}\right) \\
& =\prod_{\vartheta} \frac{1}{1-\chi_{v}\left(\check{\vartheta}\left(\varpi_{v}\right)\right) q_{v}^{-\left(s_{\vartheta}-\kappa_{\vartheta}\right) \ell(\vartheta)}} .
\end{aligned}
$$

With Proposition 2.10 in mind, we proceed as follows. Let $\sigma=\left(\Re\left(s_{\alpha}\right)\right)_{\alpha}$. Observe that in the definition $b_{v}$ we may assume $\mathbf{a} \neq \underline{0}$. Since for each $v \notin S$,

$$
\{\mathbf{a} \mid \mathbf{a} \neq \underline{0}\}=\bigcup_{\vartheta \in \Delta\left(\mathrm{G}^{\prime}\left(F_{v}\right), \mathrm{S}_{v}^{\prime}\left(F_{v}\right)\right)}\left\{\mathbf{a} ; a_{\vartheta}^{v} \neq 0\right\},
$$


we have

$$
\begin{aligned}
\sum_{v \notin S}\left|b_{v}\right| & \leq \sum_{v \notin S} \sum_{\vartheta} \sum_{a_{\vartheta} \neq 0} q_{v}^{-\langle\mathbf{a}, \sigma\rangle_{v}}\left|\left(\operatorname{vol}\left(\mathrm{K}_{v} t_{v}(\mathbf{a}) \mathrm{K}_{v}\right)-\delta\left(t_{v}(\mathbf{a})\right)\right)\right| \\
& \left.\ll \sum_{v \notin S} q_{v}^{-1} \sum_{\vartheta} \sum_{a_{\vartheta} \neq 0} q_{v}^{-\langle\mathbf{a}, \sigma\rangle_{v}} \delta\left(t_{v}(\mathbf{a})\right) \quad \text { (by Section } \underline{6.8}\right) \\
& =\sum_{v \notin S} q_{v}^{-1} \sum_{\vartheta}\left(\sum_{a_{\vartheta}=1}^{\infty} q_{v}^{-\left(\sigma_{\vartheta}-\kappa_{\vartheta}\right) a_{\vartheta} \ell(\vartheta)}\right) \prod_{\beta \neq \vartheta}\left(\sum_{a_{\beta}=0}^{\infty} q_{v}^{-\left(\sigma_{\beta}-\kappa_{\beta}\right) a_{\beta} \ell(\beta)}\right) \\
& =\sum_{v \notin S} q_{v}^{-1} \sum_{\vartheta} \frac{q_{v}^{-\left(\sigma_{\vartheta}-\kappa_{\vartheta}\right) \ell(\vartheta)}}{\prod_{\beta}\left(1-q_{v}^{-\left(\sigma_{\beta}-\kappa_{\beta}\right) \ell(\beta)}\right)} \\
& \ll \sum_{\vartheta} \sum_{v \notin S} q_{v}^{-\frac{3}{2}}<\infty .
\end{aligned}
$$

We need to show the existence of a $C>0$ such that $\left|1+a_{v}\right| \geq C>0$ for all $v$. For this

$$
\left|1+a_{v}\right| \geq \prod_{\vartheta} \frac{1}{1+q_{v}^{-\sigma_{\vartheta}+\kappa_{\vartheta}}} \geq \prod_{\vartheta} \frac{1}{2} \geq \frac{1}{2^{r}},
$$

with $r=\left|\Delta\left(G^{s p}, \mathrm{~T}^{s p}\right)\right|$. In fact, $q_{v}^{-\sigma_{k}+\kappa_{\vartheta_{k}}} \leq q_{v}^{-\frac{1}{2}}<1$.

Note that for $\mathbf{s} \in \mathcal{T}_{-\epsilon}$ the estimates are uniform, i.e., the quotient

$$
\frac{\prod_{v \notin S} \mathcal{J}_{v}(\chi)}{\prod_{v \notin S}\left(1+a_{v}\right)}
$$

is holomorphic in $\mathcal{T}_{-\epsilon}$. This finishes the proof of the theorem.

7.2. Integrals from infinite-dimensional representations. Let $\pi=\bigotimes_{v} \pi_{v}$ be an infinite-dimensional automorphic representation of $G$ which is not the automorphic representation associated to an Eisenstein series when $\mathrm{G}=\mathrm{PGL}_{2}$ (this exceptional case was treated in [37]). For $v \notin S_{F}$, let $\varphi_{v, \pi}$ be the normalized spherical function associated to $\pi_{v}$ and set $S=S_{F}$.

Theorem 7.2. Let $c>0$ be as in Theorem 4.5. The infinite product

$$
\mathcal{J}_{S}(\mathbf{s}, \pi):=\prod_{v \notin S} \int_{\mathrm{G}\left(F_{v}\right)} \varphi_{v, \pi}\left(g_{v}\right) H_{v}\left(\mathbf{s}, g_{v}\right)^{-1} d g_{v}
$$

is holomorphic for $\mathbf{s} \in \mathcal{T}_{-c}$.

Proof. We use the notation of Section 7.1. Set

$$
\mathcal{J}_{v}(\mathbf{s}, \pi)=\int_{\mathrm{G}\left(F_{v}\right)} \varphi_{v, \pi}\left(g_{v}\right) H_{v}\left(\mathbf{s}, g_{v}\right)^{-1} d g_{v} .
$$

By Cartan decomposition,

$$
\mathcal{J}_{v}(\mathbf{s}, \pi)=\sum_{\mathbf{a}} \varphi_{v, \pi}\left(t_{v}(\mathbf{a})\right) H_{v}\left(\mathbf{s}, t_{v}(\mathbf{a})\right)^{-1} \operatorname{vol}\left(\mathrm{K}_{v} t_{v}(\mathbf{a}) \mathrm{K}_{\mathbf{v}}\right) .
$$

Let

$$
a_{v}=\sum_{\vartheta} q_{v}^{-s_{\vartheta}} \operatorname{vol}\left(\mathrm{K}_{v} \check{\vartheta}\left(\varpi_{v}\right) \mathrm{K}_{v}\right) \varphi_{v, \pi}\left(\check{\vartheta}\left(\varpi_{v}\right)\right)
$$


and

$$
b_{v}=\mathcal{J}_{v}(\mathbf{s}, \pi)-1-a_{v} .
$$

Fix an $\epsilon<\frac{1}{2}$. We claim that there is a set $S^{\prime}$ of places of $F$ and a positive constant $C$ such that $\left|1+a_{v}\right| \geq C$ for all $v \notin S^{\prime}$. Since $\left|1+a_{v}\right| \geq 1-\left|a_{v}\right|$, we only need to show that $\left|a_{v}\right|$ is asymptotically bounded away from 1 . To see this we use the fact that $\left\|\varphi_{v, \pi}\right\|_{L^{\infty}} \leq 1$. Hence for $v$ outside a growing finite set $S^{\prime}$

$$
\left|a_{v}\right| \leq \sum_{\vartheta} q_{v}^{-\sigma_{\vartheta}+\kappa_{\vartheta}} \rightarrow 0
$$

for $\mathbf{s} \in \mathcal{T}_{-\epsilon}$. This implies that one can choose $S^{\prime}$ such that if $v \notin S^{\prime}$ and $\mathbf{s} \in \mathcal{T}_{-\epsilon}$, then $\left|1+a_{v}\right| \geq C$, for a constant $C$ that depends only on $\epsilon$.

Next, we prove that $\sum_{v} b_{v}$ is absolutely and uniformly convergent on $\mathcal{T}_{-\epsilon}$. Denote by $b(m)_{v}$ the set of terms such that $\mathbf{a}^{v}$ has exactly $m$ non-zero coordinates. We have

$$
\sum_{v}\left|b(1)_{v}\right| \leq \sum_{\vartheta} \sum_{v \notin S} \sum_{a_{\vartheta} \geq 2} q_{\vartheta}^{-\sigma_{\vartheta} a_{\vartheta}+\kappa_{\vartheta} a_{\vartheta}}
$$

Note that $a_{\vartheta} \neq 1$, because otherwise the term would appear in $a_{v}$. This implies that

$$
\begin{aligned}
\sum_{v}\left|b(1)_{v}\right| & \leq \sum_{\vartheta} \sum_{v \notin S} \frac{q_{v}^{-2 \sigma_{\vartheta}+2 \kappa_{\vartheta}}}{1-q_{v}^{-\sigma_{\vartheta}+\kappa_{\vartheta}}} \\
& \ll \sum_{\vartheta} \sum_{v \notin S} q_{v}^{-2 \sigma_{\vartheta}+2 \kappa_{\vartheta}} \\
& \ll \sum_{v \notin S} q_{v}^{-2+2 \epsilon} \\
& <\infty
\end{aligned}
$$

for $\epsilon<\frac{1}{2}$.

To continue, we fix an ordering of the elements of $\Delta\left(\mathrm{G}\left(F_{v}\right), \mathrm{S}_{v}\left(F_{v}\right)\right)$, say $\left\{\vartheta_{1}, \ldots\right.$, $\left.\vartheta_{l}\right\}$. Next we verify the claim for $\sum_{m \geq 2} \sum_{v}\left|b(m)_{v}\right|$, where

$$
b(m)_{v}=\sum_{1 \leq i_{1}<i_{2}<\ldots i_{m} \leq l} \sum_{a_{i_{1}}>0} \sum_{a_{i_{2}}>0} \cdots \sum_{a_{i_{m}}>0} q_{v}^{-\sum_{j=1}^{m} a_{i_{j}} s_{i_{j}}} \operatorname{vol}\left(\mathrm{K}_{v} t_{\mathbf{a}}^{\mathbf{m}} \mathrm{K}_{v}\right) \varphi_{v, \pi}\left(t_{\mathbf{a}}^{\mathbf{m}}\right) .
$$

Here $t_{\mathbf{a}}^{\mathbf{m}}$ corresponds to the vector with coordinates $a_{i_{j}}$ at $i_{j}$. Next

$$
\begin{aligned}
\left|b(m)_{v}\right| & \leq \sum_{1 \leq i_{1}<i_{2}<\ldots i_{m} \leq l} \sum_{a_{i_{1}}>0} \sum_{a_{i_{2}}>0} \cdots \sum_{a_{i_{m}}>0} q_{v}^{-\sum_{j=1}^{m} a_{i_{j}}\left(\sigma_{i_{j}}-\kappa_{\alpha_{i_{j}}}\right)} \\
& \leq \sum_{1 \leq i_{1}<i_{2}<\ldots i_{m} \leq l} \prod_{j=1}^{m} \frac{q_{v}^{-\sigma_{i_{j}}+\kappa_{\alpha_{i_{j}}}}}{1-q_{v}^{-\sigma_{i_{j}}+\kappa_{\alpha_{i_{j}}}}} \\
& \ll \sum_{1 \leq i_{1}<i_{2}<\ldots i_{m} \leq l} \prod_{j=1}^{m} q_{v}^{-\sigma_{i_{j}}+\kappa_{\alpha_{i_{j}}}} \\
& <\sum_{1 \leq i_{1}<i_{2}<\ldots i_{m} \leq l} q_{v}^{m(-1+\epsilon)} \\
& \ll q_{v}^{m(-1+\epsilon)} .
\end{aligned}
$$


Since $m \geq 2$ and $\epsilon<\frac{1}{2}$, the series $\sum_{v} q_{v}^{m(1-\epsilon)}$ is convergent, and we are done.

The holomorphy of (7.8) follows from the uniform convergence of

$$
\Sigma_{S}=\sum_{v \notin S}\left|a_{v}(\mathbf{s})\right|
$$

on compact subsets of $\mathcal{T}$, which we now establish. Fix an $\epsilon^{\prime}>0$, and suppose that $\mathbf{s} \in \mathcal{T}_{-c+\epsilon^{\prime}}$. Then

$$
\begin{aligned}
\Sigma_{S} & \leq \sum_{v \notin S} \sum_{\vartheta} q_{v}^{-\sigma_{\vartheta}} \operatorname{vol}\left(\mathrm{K}_{v} \check{\vartheta}\left(\varpi_{v}\right) \mathrm{K}_{v}\right)\left|\varphi_{v}\left(\check{\vartheta}\left(\varpi_{v}\right)\right)\right| \\
& \ll \sum_{v \notin S} \sum_{\vartheta} q_{v}^{-\sigma_{\vartheta}-c} \delta\left(\check{\vartheta}\left(\varpi_{v}\right)\right)
\end{aligned}
$$

by Lemma 6.10 and Theorem 4.5 . Finally, since by definition

$$
\delta\left(\check{\vartheta}\left(\varpi_{v}\right)\right)=q_{v}^{\kappa_{\vartheta} \ell(\vartheta)}
$$

we conclude that

$$
\Sigma_{S} \ll \sum_{v \notin S} \sum_{\vartheta} q_{v}^{-\eta-1}<\infty,
$$

for some $\eta>0$. This last inequality completes the proof of the theorem.

Corollary 7.3 (of the proof). For all $\epsilon>0$ and all compacts $K \subset \mathcal{T}_{-c+\epsilon}$ there exists a constant $C(\epsilon, K)$ such that

$$
\left|\mathcal{J}_{S}(\mathbf{s}, \pi)\right| \leq C(\epsilon, K)
$$

for all $\pi$ as above, and all $\mathbf{s} \in K$.

Corollary 7.4. Let $\mathrm{K}$ be as in Corollary 4.1. Let $\phi$ be an automorphic form in the space of an automorphic representation $\pi$ which is right invariant under $\mathrm{K}$. For $\mathbf{s} \in \mathcal{T}_{\gg 0}$ set

$$
\mathcal{J}(\mathbf{s}, \phi):=\int_{\mathrm{G}(\mathbb{A})} H(\mathbf{s}, g)^{-1} \phi(g) d g .
$$

Then $\mathcal{J}(s, \phi)$ has an analytic continuation to a function which is holomorphic on $\mathcal{T}_{-c}$. As usual we let $\Delta$ be chosen as in the proof of Lemma 4.1 of [1], and suppose $\phi$ is an eigenfunction for $\Delta$. Define $\Lambda(\phi)$ by $\Delta \cdot \phi=\Lambda(\phi) \cdot \phi$. Then for each integer $k>0$, and all $\epsilon>0$, and every compact subset $K \subset \mathcal{T}_{-c+\epsilon}$, there exists a constant $C=C(\epsilon, K, k)$, independent of $\phi$, such that

$$
|\mathcal{J}(\mathbf{s}, \phi)| \leq C \Lambda(\phi)^{-k}|\phi(e)|
$$

for all $\mathbf{s} \in K$.

Proof. Combine Corollary 7.3, Corollary 4.1, Theorem 6.7 and its corollary.

Remark 7.5. The value of the spherical function at the element $\check{\vartheta}\left(\varpi_{v}\right)$, for $\vartheta=$ $r_{v}\left(\iota^{*}(\alpha)\right)$, is related to the trace of the dominant weight $\omega_{\alpha}$ of the $L$-group applied to the Langlands class of $\pi_{v}$, and the Euler product $\mathcal{J}_{S}(\mathbf{s}, \pi)$ is regularized by a product of $L_{S}\left(s_{\alpha}, \pi, \omega_{\alpha}\right)$. Additional information about these $L$-functions would lead to better error terms in asymptotics of rational points. 


\section{Height Zeta FunCtion}

8.1. The zeta function. The main tool in the study of distribution properties of rational points is the height zeta function, defined on $\mathrm{G}(F) \backslash \mathrm{G}(\mathbb{A})$ by

$$
\mathcal{Z}(\mathbf{s}, g):=\sum_{\gamma \in \mathrm{G}(F)} H(\mathbf{s}, \gamma g)^{-1} .
$$

Proposition 8.1. The series defining $\mathcal{Z}(\mathrm{s}, g)$ converges absolutely to a holomorphic function for $\mathbf{s} \in \mathcal{T}_{\gg 0}$. In its region of convergence

$$
\mathcal{Z}(\mathbf{s}, g) \in C^{\infty}(\mathrm{G}(F) \backslash \mathrm{G}(\mathbb{A})) .
$$

Furthermore, $\mathcal{Z}(\mathbf{s}, g)$ and all of its group derivatives are in $\mathrm{L}^{2}$.

Proof. It suffices to prove the absolute convergence of $\mathcal{Z}(\mathbf{s}, g)$ for $\Re(s)$ contained in some open cone. This is a general fact (see Proposition 4.4 in [15]): since $X$ is projective the cone generated by ample classes is open in $\operatorname{Pic}(X)_{\mathbb{R}}$. Fix some ample classes $L_{j}$ generating $\operatorname{Pic}(X)$. The restriction $\mathcal{Z}\left(s L_{j}, 1\right)$ converges for $\Re(s)>\sigma_{j}$, for some $\sigma_{j}>0$. Now use the exponential property of heights. The proof of the last statement is identical to the proof of Proposition 2.3. of [37].

Proposition 8.2. The function $F(g)=\mathcal{Z}(\mathbf{s}, g)$ satisfies the conditions of Lemma 3.1, and hence has a spectral expansion.

Proof. Obvious from the proof of Theorem 7.1

By Lemma 3.1 the zeta function has an expansion of the form

$$
\mathcal{Z}(\mathbf{s}, g)=S_{1}(\mathcal{Z}(\mathbf{s}, \cdot), g)+S_{2}(\mathcal{Z}(\mathbf{s}, \cdot), g) .
$$

Since the two sides are continuous functions of $g$, we may set $g=e$ to get

$$
\mathcal{Z}(\mathbf{s})=S_{1}(\mathcal{Z}(\mathbf{s}, \cdot), e)+S_{2}(\mathcal{Z}(\mathbf{s}, \cdot), e) .
$$

We use this expansion to determine the analytic behavior of the height zeta function. The idea is to separate out the contribution of one-dimensional representations. Since the definition of $S_{1}$ involves the Laplace operator, the contribution of onedimensional representations to this term will cancel (we will momentarily see that because of the uniform convergence of the inner sum the $\Delta^{n}$ can be moved to the Eisenstein series). It remains to treat the contribution to $S_{2}$. Since both sums are of the shape considered in (3.26), without restriction of generality, we set

$$
\begin{aligned}
S^{b}(\mathbf{s})=\sum_{\chi \in \mathcal{X}} \sum_{\mathrm{P}} n & (\mathrm{~A})^{-1} \\
& \times \int_{\Pi(\mathrm{M})} \int_{\mathrm{G}(\mathbb{A})}\left(\sum_{\phi \in \mathcal{B}_{\mathrm{P}}(\pi)_{\chi}} E(x, \phi) \overline{E(y, \phi)}\right) H(\mathbf{s}, y)^{-1} d y d \pi,
\end{aligned}
$$

where the symbol $b$ indicates that the summation is over those classes which do not correspond to one-dimensional representations. The fact that the innermost sum is uniformly convergent for $y$ in compact sets is included in the first half of the proof of Lemma 4.4 of [1]. (Note that here too one needs to use Lemma 4.1 
of [1].) Therefore, we may interchange the innermost summation with the integral over $\mathrm{G}(\mathbb{A})$ to obtain

$$
\begin{aligned}
S^{b}(\mathbf{s})=\sum_{\chi \in \mathcal{X}}^{b} \sum_{\mathrm{P}} n & \\
& \times \int_{\Pi(\mathrm{M})}\left(\sum_{\phi \in \mathcal{B}_{\mathrm{P}}(\pi)_{\chi}} E(e, \phi) \int_{\mathrm{G}(\mathbb{A})} \overline{E(y, \phi)} H(\mathbf{s}, y)^{-1} d y\right) d \pi .
\end{aligned}
$$

Theorem 8.3. The function $S^{b}$ has an analytic continuation to a function which is holomorphic on $\mathcal{T}_{-c}$.

Proof. For simplicity we assume that the height function is invariant under right and left translation by the compact subgroup $\mathrm{K}$ as in Corollary 4.1, Keeping notation of Corollary 7.4, we write

$$
S^{b}(\mathbf{s})=\sum_{\chi \in \mathcal{X}}^{b} \sum_{\mathrm{P}} n(\mathrm{~A})^{-1} \int_{\Pi(\mathrm{M})}\left(\sum_{\phi \in \mathcal{B}_{\mathrm{P}}(\pi)_{\chi}} E(e, \phi) \mathcal{J}(\mathbf{s}, E(\phi, \cdot))\right) d \pi .
$$

We now use the analytic continuation and bounds established in Corollary 7.4 to obtain the analytic continuation of $S^{b}$. Let $K$ be a compact subset of $\mathcal{T}_{-c}$. Then there is an $\epsilon>0$ such that $K \subset \mathcal{T}_{-c+\epsilon}$. By Corollary 7.4, we know that for $\mathbf{s} \in K$ and all $k$ the expression

$$
\sum_{\chi \in \mathfrak{X}}^{b} \sum_{\mathrm{P}} n(\mathrm{~A})^{-1} \int_{\Pi(\mathrm{M})}\left(\sum_{\phi \in \mathcal{B}_{\mathrm{P}}(\pi)_{\chi}}|E(e, \phi)| \cdot|\mathcal{J}(\mathbf{s}, E(\phi, \cdot))|\right) d \pi
$$

is bounded by

$$
C(\epsilon, K, k) \sum_{\chi \in \mathcal{X}}^{b} \sum_{\mathrm{P}} n(\mathrm{~A})^{-1} \int_{\Pi(\mathrm{M})}\left(\sum_{\phi \in \mathcal{B}_{\mathrm{P}}(\pi)_{\chi}} \Lambda(\phi)^{-k}|E(e, \phi)|^{2}\right) d \pi .
$$

The convergence of the last expression is a consequence of Proposition 3.5. This establishes Theorem 8.3 .

Remark 8.4. In the anisotropic situation, the desired analytic properties follow from the analytic continuation of the spectral zeta function of the Laplace operator on the corresponding compact quotient.

8.2. Now we let $\underline{a}=\left(a_{\alpha}\right) \in \mathcal{T}_{\mathbb{N}}$, and for $s \in \mathbb{C}$, we set

$$
\mathcal{Z}_{\underline{a}}(s)=\mathcal{Z}\left(s\left(a_{1}, a_{2}, \ldots, a_{r}\right)\right)
$$

as a function of one complex variable. We need to determine the rightmost pole of $\mathcal{Z}_{\underline{a}}(s)$. Set

$$
\sigma(\underline{a})=\max _{i} \frac{1+\kappa_{\alpha}}{a_{\alpha}}
$$

and let $S(\underline{a})$ be the set of $\alpha$, modulo Galois action, for which the maximum is achieved, and $m(\underline{a})=\# S(\underline{a})$. The theorem implies that $\mathcal{Z}_{\underline{a}}(s)$ has no pole for $\Re(s)>\sigma(\underline{a})$. The order of pole of

$$
\int_{\mathrm{G}(\mathbb{A})} H(s \underline{a}, g)^{-1} d g
$$


at $s=\sigma(\underline{a})$ is equal to $m(\underline{a})$. Therefore, we need those automorphic characters $\chi$ such that $\xi_{\alpha}(\chi)=1$ for all $\alpha \in S(\underline{a})$. Clearly, we are interested only in those automorphic $\chi$ which satisfy

$$
\int_{\mathrm{G}(\mathbb{A})} H(s, g)^{-1} \chi(g) d g \neq 0
$$

for some $s$ in the domain of absolute convergence. This implies that $\chi$ is right, and in this case also left, invariant under the compact open subgroup $\mathrm{K}$ of $\mathrm{G}\left(\mathbb{A}_{f}\right)$. Let $\mathfrak{X}(\underline{a})$ be the collection of all such characters. By Lemma 3.1 of [21], the set $\mathfrak{X}(\underline{a})$ is finite. The proof of the above theorem shows that

Theorem 8.5. The complex function $\mathcal{Z}_{\underline{a}}(s)$ has a meromorphic continuation to $\Re(s)>\sigma(\underline{a})-\epsilon, \epsilon>0$, with an isolated pole at $\sigma(\underline{a})$ of order $m(\underline{a})$. Furthermore,

$$
\lim _{s \rightarrow \sigma(\underline{a})}(s-\sigma(\underline{a}))^{m(\underline{a})} \mathcal{Z}_{\underline{a}}(s)=\lim _{s \rightarrow \sigma(\underline{a})}(s-\sigma(\underline{a}))^{m(\underline{a})} \sum_{\chi \in \mathfrak{X}(\underline{a})} \int_{G(\mathbb{A})} H(s, g)^{-1} \chi(g) d g .
$$

The limit is a positive real number.

Proof. If we set

$$
\mathrm{G}_{\underline{a}}=\bigcap_{\chi \in \mathfrak{X}(\underline{a})} \operatorname{ker}(\chi)
$$

then the above limit is equal to

$$
\lim _{s \rightarrow \sigma(\underline{a})}(s-\sigma(\underline{a}))^{m(\underline{a})} \int_{\mathrm{G}_{\underline{a}}} H(s, g)^{-1} d g .
$$

Again by Lemma 3.1 of [21], we know that the group $G_{\underline{a}}$ has finite index in $G(\mathbb{A})$. This easily implies that the above limit is a positive number.

A special case of particular interest is when $a_{\alpha}=\kappa_{\alpha}+1$, for all $\alpha$. Because of its relevance to the anticanonical class of the wonderful compactification (cf. Proposition (5.2), we denote this $\underline{a}$ by $\underline{\kappa}$. In this case, $\sigma(\underline{\kappa})=1$, and $m(\underline{\kappa})$ is equal to the number of distinct Galois orbits in $\Delta(\mathrm{G}, \mathrm{T})$.

Proposition 8.6. Suppose $\chi$ is an automorphic character such that $\xi_{\alpha}(\chi)=1$ for all $\alpha$. Then $\chi \equiv 1$.

Proof. This easily follows from Proposition 2.9, Cartan decomposition, and the weak approximation.

The proposition shows that $\mathfrak{X}(\underline{\kappa})=\{1\}$.

Theorem 8.7. The complex function $\mathcal{Z}_{\underline{\kappa}}(s)$ has a meromorphic continuation to $\Re(s)>1-\epsilon$ for some $\epsilon>0$ with an isolated pole of order equal to the number of Galois orbits in $\Delta(\mathrm{G}, \mathrm{T})$ at $s=1$. Furthermore,

$$
\lim _{s \rightarrow 1}(s-1)^{m(\underline{\kappa})} \mathcal{Z}_{\underline{\kappa}}(s)=\lim _{s \rightarrow 1}(s-1)^{m(\underline{\kappa})} \int_{G(\mathbb{A})} H(s \underline{\kappa}, g)^{-1} d g .
$$




\subsection{Examples.}

Example 8.8 $\left(\mathrm{PGL}_{n}\right)$. Let $F_{v}$ be a local field, $\mathrm{G}=\mathrm{PGL}_{n}$ and $\chi_{v}$ a one-dimensional representation of $\mathrm{G}\left(F_{v}\right)$. Then $\chi_{v}$ has the form

$$
\chi_{v}(g)=\xi_{v}(\operatorname{det}(g))
$$

where $\xi_{v}$ is a character of $F_{v}^{*}$ whose order divides $n$. In the global situation,

$$
\chi(g)=\xi(\operatorname{det}(g)), \quad g \in \mathrm{G}(\mathbb{A}),
$$

with $\xi$ of order dividing $n$, and $\left.\xi\right|_{F^{*}}=1$. Let $\alpha_{1}, \ldots, \alpha_{n-1}$ be the simple roots, with the convention that

$$
\alpha_{i}\left(\operatorname{diag}\left(a_{1}, \ldots, a_{n}\right)\right)=a_{i} / a_{i+1}
$$

and

$$
\check{\alpha}_{i}(t)=\left(\begin{array}{cc}
t I_{i} & \\
& I_{n-i}
\end{array}\right) .
$$

It is not hard to see that

$$
2 \rho=\sum_{i=1}^{n-1} i(n-i) \alpha_{i} .
$$

Let $\underline{a}=\left(a_{1}, \ldots, a_{n-1}\right) \in \mathbb{N}^{n-1}$. We define $\sigma(\underline{a}), S(\underline{a}), m(\underline{a})$, and $\mathfrak{X}(\underline{a})$ as above. Suppose $\chi \in \mathfrak{X}(\underline{a})$. Then if $\chi=\xi$ o det, we must have

$$
\xi^{i}=1
$$

for all $i \in S(\underline{a})$. In particular, if we set

$$
d(\underline{a})=\operatorname{gcd}(n, \underset{i \in S(\underline{a})}{\operatorname{gcd}} i),
$$

then we obtain that $\chi \in \mathfrak{X}(\underline{a})$, if and only if $\chi^{d(\underline{a})}=1$.

Example $8.9\left(\mathrm{PGL}_{4}\right)$. Here

$$
2 \rho(\operatorname{diag}(a, b, c, d))=c^{3} b c^{-1} d^{-3}=\alpha^{3} \beta^{4} \gamma^{3},
$$

if $\Delta=\{\alpha=a / b, \beta=b / c, \gamma=c / d\}$. Thus $\underline{a}_{-K}=(4,5,4)$. For $\underline{a}=(1,1,1)$ we have

$$
\sigma(\underline{a})=\max (4,5,4)=5 \text { and } S(\underline{a})=\{\beta\} \text {. }
$$

We have

$$
\check{\beta}(\varpi)=\operatorname{diag}(\varpi, \varpi, 1,1) \quad \text { and } \quad \chi_{v}(\operatorname{diag}(\varpi, \varpi, 1,1))=\xi_{v}\left(\varpi^{2}\right) .
$$

Let $\xi$ have order two. By our analysis, $\chi=\xi \circ \operatorname{det}$ contributes to the asymptotic constant.

Example 8.10 $\left(\mathrm{PGU}_{3}\right)$. Let $E / F$ be a quadratic extension and $\mathrm{GU}_{3} \subset \mathrm{GL}_{3}(E)$ the set of all $g$ such that

$$
{ }^{T} g^{\sigma} S g=\lambda S, \quad \text { where } S=\left(\begin{array}{lll} 
& & 1 \\
& 1 & \\
1 & &
\end{array}\right) .
$$

Let $\mathrm{G}=\mathrm{PGU}_{3}$ and write $\lambda=\nu(g)$ for the similitude norm of $g \in \mathrm{G}$. Then

$$
\mathrm{G}^{s c}=\mathrm{SU}_{3}, \mathrm{Z}=\mathrm{Z}^{s c}=\left\{\zeta \cdot I_{3}, \zeta^{3}=1\right\} .
$$


The Galois action on $\mathrm{G}^{s c}$ is given by

$$
\tilde{\rho}(g)=S^{T} g \rho S^{-1}
$$

if $\rho \in \Gamma_{\bar{F} / F}$ restricts to $\sigma \in \Gamma_{E / F}$ and $\tilde{\rho}(g)=g^{\rho}$ if $\rho \in \Gamma_{\bar{F} / E}$.

We claim that $j: \mathrm{T}^{s c}\left(F_{v}\right) \rightarrow \mathrm{T}\left(F_{v}\right)$ is not surjective.

We have

$$
\mathrm{T}^{s c}\left(F_{v}\right)=\left\{\operatorname{diag}(a, \epsilon, a) \quad a \in E_{w}^{*}\right\}
$$

and $a a^{-\sigma} \epsilon=1$, with $\mathrm{N}(\epsilon)=1$, whereas

$$
\mathrm{T}\left(F_{v}\right)=\left\{\operatorname{diag}\left(a, b, \lambda a^{-\sigma}\right), \text { modulo scalars }\right\}
$$

with $\lambda=b^{\sigma} b$. We can take $t \in \mathrm{T}\left(F_{v}\right)$ to have the form

$$
\operatorname{diag}\left(a, 1, a^{-\sigma}\right) .
$$

Finally,

$$
j\left(\operatorname{diag}\left(a, 1, a^{-\sigma}\right)\right)=\operatorname{diag}\left(a \epsilon^{-1}, 1, a^{-\sigma} \epsilon^{\sigma}\right)=\operatorname{diag}\left(u, 1, u^{-\sigma}\right),
$$

modulo scalars. One-dimensional automorphic representations of $G(\mathbb{A})$ are given by

$$
\chi(g)=\xi(\nu(g)) .
$$

We need to consider the range of $\nu$.

If $v$ remains prime in $E$, then

$$
{ }^{T} g_{v}^{\sigma} S g_{v}=\lambda_{v} \cdot S
$$

which implies $\lambda_{v}^{3}=\mathrm{N}_{w / v}\left(\operatorname{det}\left(g_{v}\right)\right)$. Thus $\lambda_{v} \in \mathrm{N}_{w / v}\left(E_{w}^{*}\right)$. If $v$ splits in $E$, then there is no condition on $\lambda_{v}$. Thus

$$
\nu: \mathrm{G}(\mathbb{A}) \rightarrow \mathrm{N}_{E / F}\left(\mathbb{A}_{E}^{*}\right) .
$$

Also $\nu\left(\alpha_{v} \cdot I_{3}\right)=\mathrm{N}_{W / V}\left(\alpha_{v}\right)$ if $v$ remains prime in $E$.

We may take for the adjoint group $\mathrm{U}_{3} / \mathrm{Z}$, where $\mathrm{Z}=\operatorname{diag}(\epsilon, \epsilon, \epsilon)$ and $\mathrm{N}_{E / F}(\epsilon)=$ 1. We have $\chi(g)=\xi(\operatorname{det}(g)), g \in \mathrm{G}(\mathbb{A})$. Here $\xi \in \mathbb{N}^{1}(\mathbb{A}) / \mathbb{N}^{1}(F), \xi^{3}=1$. By Hasse's theorem,

$$
\begin{array}{cl}
\mathbb{G}_{m}\left(\mathbb{A}_{E}\right) & \rightarrow \mathbb{N}^{1}(\mathbb{A}), \\
\mathbb{G}_{m}(E) & \rightarrow \mathbb{N}^{1}(E), \\
x & \mapsto x \cdot x^{-\sigma}
\end{array}
$$

are surjective. We may view $\xi$ as a character $\eta$ of $\mathbb{G}_{m}\left(\mathbb{A}_{E}\right) / \mathbb{G}_{m}(E)$ with $\eta^{3}=1$. The map

$$
j: \mathrm{T}^{s c}\left(F_{v}\right) \rightarrow \mathrm{T}\left(F_{v}\right)
$$

is not surjective, since $\mathrm{T}\left(F_{v}\right)$ is the set of all $\operatorname{diag}\left(a, 1, a^{-\sigma}\right)$ and $\mathrm{T}^{s c}\left(F_{v}\right)$ the set of $\operatorname{diag}\left(a, \epsilon, a^{-\sigma}\right)$, with $a a^{-\sigma} \epsilon=1$.

We wish to define

$$
L(s, \chi)=L_{E}(s, \eta)
$$

This leads to the necessity of introducing a norm map. In this example we have

$$
\begin{aligned}
\mathrm{N}_{w / v}: \mathrm{T}\left(E_{w}\right) & \rightarrow \mathrm{T}\left(F_{v}\right), \\
t & \mapsto t \cdot \tilde{\sigma}(t)
\end{aligned}
$$

with $\tilde{\sigma}(t)=S t^{\sigma} S^{-1}$. Thus

$$
\mathrm{N}_{w / v}(\operatorname{diag}(a, b, c))=\operatorname{diag}\left(a c^{-\sigma}, b b^{-\sigma}, c a^{-\sigma}\right) .
$$


Note that

$$
1 \rightarrow \mathrm{SU}_{v} / \mu_{3} \rightarrow \mathrm{U}_{3} / \mathrm{Z} \stackrel{\operatorname{det}}{\longrightarrow} \mathbb{N}_{v}^{1} /\left(\mathbb{N}_{v}^{1}\right)^{3} \rightarrow 1
$$

is exact.

\section{MANin's COnJeCture}

In this section we apply Theorem 8.7 to obtain a result regarding the number of rational points of bounded height on wonderful compactifications of the group G.

We first recall a Tauberian theorem ([14], Appendix A):

Theorem 9.1. Let $\left(\lambda_{n}\right)_{n \in \mathbb{N}}$ be an increasing sequence of positive real numbers, and $\left(r_{n}\right)_{n \in \mathbb{N}}$ a sequence of positive real numbers. Suppose the Dirichlet series $f(s)$ defined by

$$
f(s)=\sum_{n=0}^{\infty} \frac{r_{n}}{\lambda_{n}^{s}}
$$

satisfies the following conditions:

- the series $f$ converges in some right half plane $\Re(s)>a>0$;

- the series $f$ has a meromorphic continuation to a half plane $\Re(s)>a-\delta_{0}>$ 0 ;

- in this region, it has a unique pole at $s=a$ with multiplicity $b \in \mathbb{N}$. Set $c=\lim _{s \rightarrow a} f(s)(s-a)^{b}>0$;

- furthermore, there is a $d>0$ such that for $\Re(s)>a-\delta_{0}$ we have the estimate

$$
\left|f(s) \frac{(s-a)^{b}}{s^{b}}\right|=O\left(|1+\Im(s)|^{d}\right) .
$$

Then there is a monic polynomial $P$ of degree $b-1$ and an $\epsilon>0$ such that

$$
N(X):=\sum_{\lambda_{n} \leq B} r_{n}=\frac{c}{a(b-1) !} X^{a} P(\log (B))+O\left(X^{a-\epsilon}\right)
$$

as $B \rightarrow \infty$.

Theorem 9.2. Let $X$ be the compactification of a semi-simple group $\mathrm{G}$ of adjoint type over $F$ as in Section 5 and let $\mathcal{L}=\left(L,\|\cdot\|_{v}\right)$ be an adelically metrized line bundle such that its class $[L] \in \operatorname{Pic}(X)$ is contained in the interior of the cone of effective divisors $\Lambda_{\mathrm{eff}}(X)$. Then there is a monic polynomial $P$ of degree $b(L)-1$, and an $\epsilon>0$ such that

$$
\mathcal{N}(\mathrm{G}, \mathcal{L}, B):=\#\left\{x \in \mathrm{G}(F) \mid H_{\mathcal{L}}(x) \leq B\right\}=c(\mathcal{L}) B^{a(L)} P(\log (B))+O\left(B^{a(L)-\epsilon}\right)
$$

as $B \rightarrow \infty$. Here

$$
a(L)=\inf \left\{a \mid a[L]+\left[K_{X}\right] \in \Lambda_{\mathrm{eff}}(X)\right\}
$$

(where $K_{X}$ is the canonical line bundle of $\left.X\right), b(L)$ is the (maximal) codimension of the face of $\Lambda_{\text {eff }}(X)$ containing $a(L)[L]+\left[K_{X}\right]$ and $c(\mathcal{L})>0$. Moreover, $c\left(-\mathcal{K}_{X}\right)$ is the constant defined in 30 .

Proof. By the proof of Theorem 8.3, it suffices to concentrate on the contribution of one-dimensional representations to the spectral expansion of the height zeta function. We now recall Theorem 7.1 The assumptions of Theorem 9.1 are satisfied by well-known results on the growth of Hecke $L$-functions in vertical strips (see, e.g., Theorem 4.2.1 of [5]). Modulo the determination of the constant $c(\mathcal{L})$, the theorem is now a consequence of Theorem 8.5 as one easily checks that the numbers $\sigma(\underline{a})$ 
and $m(\underline{a})$ defined in Section 8.2 are, respectively, the numbers $a(L)$ and $b(L)$. The comparison of the constant $c\left(-\mathcal{K}_{X}\right)$ with Peyre's constant is identical to the proof of Theorem 7.2 of [6]. The key points are as follows:

- $H^{1}\left(F, \operatorname{Pic}\left(X_{\bar{F}}\right)\right)=\operatorname{Br}(X) / \operatorname{Br}(F)=1$, since $\operatorname{Pic}\left(X_{\bar{F}}\right)$ is a Galois permutation module;

- $\overline{\mathrm{G}(F)}=\mathrm{G}(\mathbb{A})$;

- $\int_{G(\mathbb{A})} \omega_{S}=\int_{X(\mathbb{A})} \omega_{S}$ (where $\omega_{S}$ is the Tamagawa measure defined in [30]);

- $\omega_{v}=\tau_{v}(\mathrm{G})^{-1} H_{-K_{X}}(\cdot) d g_{v}$, for all $v \in \operatorname{Val}(F)$.

The second fact is proved in [35]; here it is important that $\mathrm{G}$ is of adjoint type. For the identifications of the measures see $[30,7$.

\section{ACKNOWLEDGMENTS}

The authors wish to thank Erez Lapid, Hee Oh, and Peter Sarnak for various useful discussions.

\section{REFERENCES}

1. James Arthur, A trace formula for reductive groups. I. Terms associated to classes in $G(\mathbf{Q})$, Duke Math. J. 45 (1978), no. 4, 911-952. MR518111(80d:10043)

2. - A trace formula for reductive groups. II. Applications of a truncation operator, Compositio Math. 40 (1980), no. 1, 87-121. MR558260 (81b:22018)

3. . On a family of distributions obtained from Eisenstein series. I. Application of the Paley-Wiener theorem, Amer. J. Math. 104 (1982), no. 6, 1243-1288. MR681737(85k:22044)

4. V. V. Batyrev and Yu. I. Manin, Sur le nombre des points rationnels de hauteur borné des variétés algébriques, Math. Ann. 286 (1990), no. 1-3, 27-43. MR1032922 (91g:11069)

5. Victor V. Batyrev and Yuri Tschinkel, Rational points of bounded height on compactifications of anisotropic tori, Internat. Math. Res. Notices (1995), no. 12, 591-635. MR 1369408 (97a:14021)

6. , Manin's conjecture for toric varieties, J. Algebraic Geom. 7 (1998), no. 1, 15-53. MR 1620682 (2000c:11107)

7. _ Tamagawa numbers of polarized algebraic varieties, Astérisque (1998), no. 251, 299-340, Nombre et répartition de points de hauteur bornée (Paris, 1996). MR 1679843 (2000d:11090)

8. A Borel, Proceedings of Symposia in Pure Mathematics. Vol. IX: Algebraic groups and discontinuous subgroups, Proceedings of the Symposium in Pure Mathematics of the American Mathematical Society held at the University of Colorado, Boulder, Colorado (July 5-August 6), vol. 1965, American Mathematical Society, Providence, R.I., 1966. MR0202512 (34:2381)

9. A. Borel, Seminar on Algebraic Groups and Related Finite Groups. (Held at The Institute for Advanced Study, Princeton, N.J., 1968/69), Lecture Notes in Mathematics, Vol. 131, Springer-Verlag, Berlin, 1970. MR0258838(41:3484)

10. Armand Borel and Jacques Tits, Groupes réductifs, Inst. Hautes Études Sci. Publ. Math. (1965), no. 27, 55-150. MR0207712 (34:7527)

11. M. Brion, Curves and divisors in spherical varieties, Algebraic groups and Lie groups, Austral. Math. Soc. Lect. Ser., vol. 9, Cambridge Univ. Press, Cambridge, 1997, pp. 21-34. MR 1635672 (99f:14006)

12. Michel Brion, Variétés sphériques et théorie de Mori, Duke Math. J. 72 (1993), no. 2, 369-404. MR:1248677 (94j:14045)

13. W. Casselman, The unramified principal series of $\mathfrak{p}$-adic groups. I. The spherical function, Compositio Math. 40 (1980), no. 3, 387-406. MR571057 (83a:22018)

14. Antoine Chambert-Loir and Yuri Tschinkel, Fonctions zêta des hauteurs des espaces fibrés, Rational points on algebraic varieties, Progr. Math., vol. 199, Birkhäuser, Basel, 2001, pp. 71115. MR 1875171 (2003a:11079)

15. - On the distribution of points of bounded height on equivariant compactifications of vector groups, Invent. Math. 148 (2002), no. 2, 421-452. MR.1906155 (2003d:11094) 
16. C. De Concini and C. Procesi, Complete symmetric varieties, Invariant theory (Montecatini, 1982), Lecture Notes in Math., vol. 996, Springer, Berlin, 1983, pp. 1-44. MR718125 $(85 \mathrm{e}: 14070)$

17. C De Concini and T. A. Springer, Compactifications of symmetric varieties, Transform. Groups 4 (1999), no. 2-3, 273-300. MR.1712864 (2000f:14079)

18. J. Denef, On the degree of Igusa's local zeta function, Amer. J. Math. 109 (1987), no. 6, 991-1008. MR919001 (89d:11108)

19. Jens Franke, Yuri I. Manin, and Yuri Tschinkel, Rational points of bounded height on Fano varieties, Invent. Math. 95 (1989), no. 2, 421-435. MR974910 (89m:11060)

20. Ramesh Gangolli and V. S. Varadarajan, Harmonic analysis of spherical functions on real reductive groups, vol. 101, Springer-Verlag, Berlin, 1988. MR954385 (89m:22015)

21. A. Gorodnik, F. Maucourant, and H. Oh, Manin's conjecture on rational points of bounded height and adelic mixing, math.NT/0601127.

22. Benedict H. Gross, On the Satake isomorphism, Galois representations in arithmetic algebraic geometry (Durham, 1996), London Math. Soc. Lecture Note Ser., vol. 254, Cambridge Univ. Press, Cambridge, 1998, pp. 223-237. MR.1696481 (2000e:22008)

23. N. Iwahori and H. Matsumoto, On some Bruhat decomposition and the structure of the Hecke rings of p-adic Chevalley groups, Inst. Hautes Études Sci. Publ. Math. (1965), no. 25, 5-48. MR.0185016 (32:2486)

24. H. H. Kim and F. Shahidi, Functorial products for GLb2 $\times$ GLb3 and the symmetric cube for GLb2, Ann. of Math. (2) 155 (2002), no. 3, 837-893. MR1923967 (2003m:11075)

25. Martin Kneser, Starke Approximation in algebraischen Gruppen. I, J. Reine Angew. Math. 218 (1965), 190-203. MR0184945 (32:2416)

26. Erez Lapid, On the fine spectral expansion of the Jacquet's relative trace formula, J. Inst. Math. Jussieu 5 (2006), 263-308. MR.2225043 (2007d:11059)

27. C. Mœglin and J.-L. Waldspurger, Spectral decomposition and Eisenstein series, Cambridge Tracts in Mathematics, vol. 113, Cambridge University Press, Cambridge, 1995. MR 1361168 (97d:11083)

28. D. Mumford, J. Fogarty, and F. Kirwan, Geometric invariant theory, third ed., vol. 34, Springer-Verlag, Berlin, 1994. MR.1304906 (95m:14012)

29. Hee Oh, Uniform pointwise bounds for matrix coefficients of unitary representations and applications to Kazhdan constants, Duke Math. J. 113 (2002), no. 1, 133-192. MR1905394 (2003d:22015)

30. Emmanuel Peyre, Hauteurs et mesures de Tamagawa sur les variétés de Fano, Duke Math. J. 79 (1995), no. 1, 101-218. MR1340296 (96h:11062)

31. Emmanuel Peyre (ed.), Nombre et répartition de points de hauteur bornée, Paris, Société Mathématique de France, 1998, Papers from the seminars held in Paris, April/May 1996, Astérisque No. 251 (1998). MR.1679267 (99j:11001)

32. - Torseurs universels et méthode du cercle, Rational points on algebraic varieties, Progr. Math., vol. 199, Birkhäuser, Basel, 2001, pp. 221-274. MR.1875176 (2003d:11092)

33. Vladimir Platonov and Andrei Rapinchuk, Algebraic groups and number theory, Pure and Applied Mathematics, vol. 139, Academic Press Inc., Boston, MA, 1994. MR.1278263 (95b:11039)

34. Jonathan D. Rogawski, Automorphic representations of unitary groups in three variables, Annals of Mathematics Studies, vol. 123, Princeton University Press, Princeton, NJ, 1990. MR $1081540(91 \mathrm{k}: 22037)$

35. J.-J. Sansuc, Groupe de Brauer et arithmétique des groupes algébriques linéaires sur un corps de nombres, J. Reine Angew. Math. 327 (1981), 12-80. MR631309 (83d:12010)

36. Ichirô Satake, Theory of spherical functions on reductive algebraic groups over $\mathfrak{p}$-adic fields, Inst. Hautes Études Sci. Publ. Math. (1963), no. 18, 5-69. MR0195863 (33:4059)

37. Joseph Shalika, Ramin Takloo-Bighash, and Yuri Tschinkel, Rational points on compactifications of semi-simple groups of rank 1, Arithmetic of higher-dimensional algebraic varieties (Palo Alto, CA, 2002), Progr. Math., vol. 226, Birkhäuser Boston, Boston, MA, 2004, pp. 205233. MR2029871 (2004m:11104)

38. Joseph A. Shalika and Yuri Tschinkel, Height zeta functions of equivariant compactifications of the Heisenberg group, Contributions to automorphic forms, geometry, and number theory (Baltimore, MD), Johns Hopkins Univ. Press, 2004, pp. 743-771. MR2058627 (2005c:11110)

39. Allan J. Silberger, Introduction to harmonic analysis on reductive $p$-adic groups, Mathematical Notes, vol. 23, Princeton University Press, Princeton, N.J., 1979. MR544991 (81m:22025) 
40. Elisabetta Strickland, A vanishing theorem for group compactifications, Math. Ann. 277 (1987), no. 1, 165-171. MR884653 (88b:14035)

41. J. Tits, Reductive groups over local fields, Automorphic forms, representations and $L$-functions (Proc. Sympos. Pure Math., Oregon State Univ., Corvallis, Ore., 1977), Part 1, Proc. Sympos. Pure Math., XXXIII, Amer. Math. Soc., Providence, R.I., 1979, pp. 29-69. MR546588 (80h:20064)

Department of Mathematics, Johns Hopkins University, 3400 N. Charles Street, BalTIMORE, MARYland 21218-2686

E-mail address: shalika@math.jhu.edu

Department of Mathematics, Princeton University, Fine Hall, Washington Road, Princeton, New Jersey 08544-1000

E-mail address: rtakloo@math.princeton.edu

Courant Institute, NYU, 251 Mercer Street, New York, New York 10012

Current address: Mathematisches Institut, Bunsenstr. 3-5, 37073 Göttingen, Germany

E-mail address: tschinkel@cims.nyu.edu 